\title{
Modeling aerodynamically generated sound of helicopter rotors
}

\author{
Kenneth S. Brentner*,1 \\ Department of Aerospace Engineering, The Pennsylvania State University, \\ University Park, PA 16802, USA \\ and \\ F. Farassat
}

Aeroacoustics Branch, NASA Langley Research Center, Hampton, VA 23681, USA

\begin{abstract}
A great deal of progress has been made in the modeling of aerodynamically generated sound of rotors over the past decade. Although the modeling effort has focused on helicopter main rotors, the theory is generally valid for a wide range of rotor configurations. The Ffowcs Williams-Hawkings (FW-H) equation has been the foundation for much of the development. The monopole and dipole source terms of the $\mathrm{FW}-\mathrm{H}$ equation account for the thickness and loading noise, respectively. Bladevortex-interaction noise and broadband noise are important types of loading noise, hence much research has been directed toward the accurate modeling of these noise mechanisms. Both subsonic and supersonic quadrupole noise formulations have been developed for the prediction of high-speed impulsive noise. In an effort to eliminate the need to compute the quadrupole contribution, the $\mathrm{FW}-\mathrm{H}$ equation has also been utilized on permeable surfaces surrounding all physical noise sources. Comparisons of the Kirchhoff formulation for moving surfaces with the $\mathrm{FW}-\mathrm{H}$ equation have shown that the Kirchhoff formulation for moving surfaces can give erroneous results for aeroacoustic problems. Finally, significant progress has been made incorporating the rotor noise models into full vehicle noise prediction tools.
\end{abstract}

* Corresponding author.

Email addresses: ksbrentner@psu.edu (Kenneth S. Brentner), f.farassat@larc.nasa.gov (F. Farassat).

1 Formerly Senior Research Engineer, NASA Langley Research Center, Hampton, VA 23681, USA.

Preprint submitted to Progress in Aerospace Sciences 


\section{Contents}

1 Notation 3

2 Introduction 4

$3 \quad$ Background and Historical Perspective 6

$\begin{array}{lll}3.1 & \text { Physical Sources of Rotor Noise } & 6\end{array}$

$\begin{array}{lll}3.2 & \text { Early Theoretical Developments } & 7\end{array}$

3.3 The Ffowcs Williams - Hawkings Era 8

4 Theoretical Background: The Ffowcs Williams - Hawkings Equation 10

$\begin{array}{llr}4.1 & \text { Derivation } & 10\end{array}$

$\begin{array}{lll}4.2 & \text { Interpretation and Observations } & 16\end{array}$

$\begin{array}{llr}4.3 & \text { Integral Formulations } & 19\end{array}$

$5 \quad$ Numerical Algorithms for Rotor Noise Prediction 23

5.1 Retarded-Time Algorithms 24

$\begin{array}{lll}5.2 & \text { Collapsing-Sphere Algorithms } & 28\end{array}$

$\begin{array}{lll}5.3 & \text { Emission-Surface Algorithms } & 29\end{array}$

$6 \quad$ Recent Advances 31

6.1 High-Speed Impulsive Noise $\quad 31$

6.2 Blade-Vortex-Interaction (BVI) Noise 36

$\begin{array}{lll}\text { 6.3 } & \text { Broadband Noise Prediction } & 39\end{array}$

6.4 Ffowcs Williams - Hawkings Equation as a Kirchhoff Formulation 43

$\begin{array}{lll}\text { 6.5 Vehicle Noise Prediction } & 46\end{array}$

$7 \quad$ Concluding Remarks $\quad 50$

$\begin{array}{ll}\text { References } & 51\end{array}$ 


\section{Notation}

$\square^{2} \quad$ wave operator, $\square^{2}=\frac{1}{c^{2}} \frac{\partial^{2}}{\partial t^{2}}-\nabla^{2}$

$c \quad$ sound speed in quiescent medium

$f=0$ function that describes the source surface, e.g., a rotor blade

$g \quad=\tau-t+r / c$

$H(f)$ Heaviside function, $H(f)=0$ for $f<0$ and $H(f)=1$ for $f>0$

$\ell_{i} \quad$ components of local force intensity that acts on the fluid, $\ell_{i}=P_{i j} n_{j}$

$\ell_{r} \quad \ell_{i} \hat{r}_{i}$

$\dot{\ell}_{r} \quad \dot{\ell}_{i} \hat{r}_{i}$

M local Mach number vector of source with respect to a frame fixed to the undisturbed medium, with components $M_{i}$

$\dot{M}_{i} \quad \partial M_{i} / \partial \tau$

$M \quad|\mathbf{M}|$

$M_{A T}$ advancing-tip Mach number

$M_{H} \quad$ hover tip Mach number

$M_{n} \quad$ Mach number in direction normal to the surface, $M_{i} \hat{n}_{i}$

$M_{r} \quad$ Mach number of source in radiation direction, $M_{i} \hat{r}_{i}$

$\dot{M}_{r} \quad \dot{M}_{i} \hat{r}_{i}$

$\hat{\mathbf{n}} \quad$ unit outward normal vector to surface, with components $\hat{n}_{i}$

$p^{\prime} \quad$ acoustic pressure, $p-p_{o}$ in undisturbed medium; $p^{\prime} \equiv c^{2} \rho^{\prime}$ on LHS of Lighthill and in $\mathrm{FW}-\mathrm{H}$ equations

$P_{i j} \quad$ compressive stress tensor

$Q_{i j} \quad$ far-field quadrupole tensor, defined in equation (52)

$r \quad$ distance between observer and source, $r=|\mathbf{x}-\mathbf{y}|$

$\hat{\mathbf{r}} \quad$ unit vector in the radiation direction, with components $\hat{r}_{i}$;

$\hat{\mathbf{r}}=(\mathbf{x}-\mathbf{y}) / r$

$R \quad$ rotor radius

$d S \quad$ element of the rotor blade surface

$t$ observer time

$T_{i j} \quad$ Lighthill stress tensor, $\rho u_{i} u_{j}+P_{i j}-c^{2} \rho^{\prime} \delta_{i j}$

$u_{i} \quad$ components of local fluid velocity

$v_{n} \quad$ local normal velocity of source surface

$V_{H} \quad$ helicopter velocity

$v_{\dot{n}} \quad v_{i} \dot{\hat{n}}_{i}$

$\dot{v}_{n} \quad \dot{v}_{i} \hat{n}_{i}$

$\mathrm{x} \quad$ observer position vector, with components $x_{i}$

$\mathbf{y} \quad$ source position vector, with components $y_{i}$

Greek symbols:

$\delta(f)$ Dirac delta function 
$\delta_{i j} \quad$ Kronecker delta, $\delta_{i j}=1$ for $i=j$, otherwise $\delta_{i j}=0$

$d \Gamma \quad$ element of length of the $\Gamma$-curve (intersection of collapsing sphere and $f=0)$.

$\Lambda \quad \sqrt{1-2 M_{n} \cos \theta+M_{n}^{2}}$

$\mu \quad$ advance ratio

$\Omega \quad$ rotor angular velocity

$d \Omega \quad$ element of surface area of the collapsing sphere $g=0$

$\rho_{o} \quad$ density of quiescent medium

$\rho^{\prime} \quad$ density perturbation, $\rho-\rho_{o}$

$d \Sigma \quad$ element of surface area of the influence surface $F(\mathbf{y} ; \mathbf{x}, t)=f(\mathbf{y}, t-r / c)$

$\theta \quad$ angle between the normal direction $\hat{\mathbf{n}}$ and the radiation direction $\hat{\mathbf{r}}$

$\tau \quad$ source time

Subscripts:

ret quantity is evaluated at the retarded time, $\tau=t-r / c$

$o$ denotes fluid variable in quiescent medium

$L \quad$ loading noise component

$T \quad$ thickness noise component

$Q \quad$ quadrupole noise component

Note: Summation convention is used in this paper with the exception of $T_{r r}=$ $T_{i j} \hat{r}_{i} \hat{r}_{j}$ and $Q_{r r}=Q_{i j} \hat{r}_{i} \hat{r}_{j}$.

\section{Introduction}

Noise has been an undesirable byproduct of aerospace vehicles from the time of early aircraft until now. Originally, aircraft noise was not much of an issue because of the overarching requirement of improving vehicle performance. As aerospace technology has matured, more resources have been devoted to the reduction of aerodynamically generated sound. With the present level of aerospace technology maturity, both the public and regulatory bodies have focused their concern on safety, emissions and noise rather than either performance or efficiency. ${ }^{2}$ This situation is really a credit to the success of past generations of aerospace designers and engineers. The challenge is that anticipated increases in the use of air travel will also bring unacceptable increases

2 The military still focuses primarily on performance with less emphasis for noise than their civilian counterparts. Thus civil vehicles with military origins often do not have the same degree of noise control incorporated into their designs as civil rotorcraft. 
in aircraft noise - if nothing is done. Fortunately, a great deal of progress has been made both in understanding the noise-generation mechanisms and in developing first-principles models for prediction of the sound. However, the use of this knowledge in the reduction of aircraft noise has been less successful.

Rotorcraft are inherently complex aeromechanical vehicles, and hence have lagged fixed-wing aircraft in the understanding of the mechanisms responsible for rotor noise and the development of accurate and efficient prediction methods based upon the fluid physics (as opposed to empirical methods). Furthermore, fixed-wing aircraft can benefit from both source noise reduction and noise control through features such as engine duct liners. Rotorcraft do not have this luxury and must rely almost entirely on source noise reduction. A number of high quality experimental tests, concentrated national research programs and rigorous theoretical developments have greatly expanded both our understanding of rotor noise sources and our ability to predict main rotor noise. The maturation of computational fluid dynamics (CFD) and its application to rotor aerodynamics has been an enabling step necessary for accurate, first-principles noise prediction. Nevertheless, rotor aerodynamics is often one of the weak links leading to unsatisfactory acoustic computations.

In this article, the focus will be on the aerodynamically generated sound of main rotors and the recent advances in rotor noise prediction. In 1994, the authors published a status report on helicopter noise prediction [1]. That report provides a historical perspective and method assessment that is a useful backdrop for this article. The goal of the present article is two-fold: 1) to give a concise historical and theoretical background of the modeling of aerodynamically generated sound of helicopter rotors and 2) to highlight significant theoretical and computational developments that have been completed since 1994. Emphasis is placed on both theoretical development and numerical interpretation because these elements help to explain the reasons why various computational methods were selected. This article does not focus on the relative magnitude of the various noise sources even though this an important aspect of rotor noise prediction that depends both on rotor size and operating environment. Although the primary focus of the article is on source noise prediction, system noise prediction is also recognized as an important area that has also seen significant progress over the last decade. Therefore a brief discussion of rotorcraft system noise prediction is included. This article focuses primarily on the rotor noise prediction work at NASA Langley Research Center: however, an attempt has been made to include significant contributions of other researchers. 


\section{Background and Historical Perspective}

The operating environment of a helicopter rotor is extremely complex and fundamentally unsteady (as shown in figure 1). The rotor blade on a helicopter in forward flight with speed $V_{H}$ experiences high-speed flow on the advancing side of the rotor (advancing-tip speed $V_{a d v} .=\Omega R+V_{H}$ ) and significantly lower-speed flow on the retreating side of the rotor (retreating-tip speed $V_{\text {ret. }}=$ $\left.\Omega R-V_{H}\right)$. The difference in velocity on the advancing and retreating sides of the rotor is managed through lateral cyclic pitch control so that the lift of the rotor is balanced from side to side. Longitudinal cyclic pitch control is used to change the rotor tip-path plane which in turn generates thrust to propel the helicopter forward. The aerodynamic and dynamic forces acting on the rotor further lead to both out-of-plane (flapping), in-plane (lead-lag) and bending motions of the rotor blade. Finally, the rotor operates very close to its own wake, hence the wake is much more important in rotor aerodynamics than in fixed-wing or propeller aerodynamics.

[Fig. 1
about

\subsection{Physical Sources of Rotor Noise}

This complicated aeromechanical environment contributes to both discrete frequency and broadband aerodynamic noise generation through several distinct noise mechanisms. Discrete-frequency noise is usually divided into the deterministic components of thickness and loading noise, blade-vortex interaction noise $^{3}$ and high-speed impulsive noise. Similarly, broadband noise consists of the non-deterministic loading noise sources classified as turbulence ingestion noise, blade-wake interaction noise and blade self-noise. Although tail rotor noise may be subjectively even more important than main rotor noise, it has not received much attention from the noise modeling community and will not be addressed in any significant way in this article. Much of the theoretical development for main rotor noise is applicable to tail rotors, however. Other exterior noise sources, such as engine noise, and interior noise sources, such as drivetrain and gear noise, are also important rotorcraft noise sources, but they are not considered here.

Thickness noise and loading noise, known together as rotational noise, are related to linear aerodynamic theory. Thickness noise is due to the displacement of the fluid in the flow field by the rotor blade while loading noise is caused by the accelerating force on the fluid generated by moving blade surface. The term "loading noise" is usually a reference to the harmonic noise from nonimpulsive loading sources, whereas impulsive loading noise due to blade-vortex

$\overline{3}$ Blade-vortex-interaction noise is a specific type of loading noise that is extremely annoying when it occurs. 
interaction (BVI) and non-deterministic loading associated with broadband noise are treated separately.

Blade-vortex interaction noise (known earlier as blade slap) is the result of a shed tip vortex subsequently interacting with a following blade, while broadband noise is the result of blade loading associated with turbulent flow on or near the blade surface. Turbulence ingested into the rotor can produce both discrete-frequency and broadband noise. This turbulence can occur naturally in the atmosphere or come from blade wakes. Blade self-noise generation is by turbulent phenomena occurring on the blade itself. Random pressure fluctuations on the blade surface result from turbulence within attached or separated boundary layers. Other self-noise mechanisms include tip vortex formation, laminar vortex shedding and trailing edge noise.

At high advancing-tip speeds, the rotor generates an impulsive noise of high intensity with in-plane directivity. The peak level of the noise is in the region forward of the helicopter. This noise, known as high-speed impulsive noise, is associated with the transonic flow around the blade. Figure 2 shows the typical directionality of each of various types of rotor noise.

\subsection{Early Theoretical Developments}

In order to put the more recent advances in helicopter noise prediction in to a proper perspective, it is helpful to recall that by the late 1930's it was known that for rotating blades, both the blade loading and the blade thickness could generate noise by separate mechanisms. Gutin [2] obtained the first theoretical result for calculation of the level of harmonics of propeller noise due to blade loads. Demming [3] and Ernsthausen [4] worked independently on the problem of thickness noise. Garrick and Watkins [5] extended Gutin's result to propellers in forward flight in the early fifties. Most of this early work focused on propellers because the helicopter was still in its infancy.

By the 1960's the noise of helicopters became an important issue. Initially both the piston engine and the rotor were the major generators of noise, but with the introduction of the turboshaft engine, the main and tail rotors became the dominant external noise sources. It was realized that unsteady blade surface pressure fluctuations were largely responsible for the discrete and broadband noise of the rotors. However, the acoustic theories developed earlier for propellers were only applicable to hovering rotors because they did not include the unsteady blade loading. In practice, a hovering rotor has significant unsteady loading, hence, the propeller acoustics theories were inadequate even for hover.

Some of the first noise prediction theories applied specifically to helicopter 
rotors were developed by Lowson [6] and Wright [7]. Lowson and Ollerhead [8] also developed a computer code for rotor noise prediction both in the time and frequency domains. The lack of computer power at the time and the limited understanding of the role of the blade surface pressure distribution led these researchers to use compact source calculations. It is interesting to note that the thickness noise of rotors was largely a neglected subject of the 1960's. Nevertheless this period was marked by systematic experimental and theoretical study of the source mechanisms of helicopter rotors $[9,10]$.

\subsection{The Ffowcs Williams - Hawkings Era}

In 1969 Ffowcs Williams and Hawkings published their now classic paper "Sound Generation by Turbulence and Surfaces in Arbitrary Motion" [11] which generalized Lighthill's acoustic analogy approach [12] to include the effects of very general types of surfaces and motions. Using the mathematical theory of distributions (also known as generalized functions), they were able to rearrange the Navier-Stokes equations into the form of an inhomogeneous wave equation with a quadrupole source distribution in the volume surrounding the body and monopole and dipole sources on the body surface. The Ffowcs Williams - Hawkings $(\mathrm{FW}-\mathrm{H})$ equation can be written in differential form ${ }^{1}$ as

$$
\square^{2} p^{\prime}(\mathbf{x}, t)=\frac{\bar{\partial}}{\partial t}\left[\rho_{o} v_{n} \delta(f)\right]-\frac{\bar{\partial}}{\partial x_{i}}\left[\ell_{i} \delta(f)\right]+\frac{\bar{\partial}^{2}}{\partial x_{i} \partial x_{j}}\left[T_{i j} H(f)\right]
$$

where $v_{n}$ is the local velocity of the body in the direction normal to the surface implicitly defined by $f=0, l_{i}$ are the components of the local force on the surface, $T_{i j}$ is the Lighthill stress tensor and $\delta(f)$ and $H(f)$ are the Dirac delta and Heaviside functions, respectively. The beauty of the generalized function approach is that all of the manipulations are completely rigorous and ad hoc reasoning is not required.

The effect of Ffowcs Williams and Hawkings paper was to encourage theoretical work on helicopter rotor noise in the 1970's. Hawkings and Lowson [13] and Farassat [14-16] were among the first groups of researchers applying the FW-H equation to the problem of rotor noise prediction. Thickness noise was proposed as the source for the phenomenon of high-speed blade slap, now known as high-speed impulsive noise. Later Schmitz and Yu [17] and Hanson and Fink [18] discovered that this conclusion was only partially correct. The

$\overline{1}$ Ffowcs Williams and Hawkings actually wrote this equation in terms of the density perturbation, $\rho^{\prime}$. Following the notation used by Farassat, $p^{\prime}$ is used on the left side of the equation rather than $c^{2} \rho^{\prime}$. While $p^{\prime}$ differs from the acoustic pressure near the source region, outside the source region linear wave propagation is expected, hence $p^{\prime}=c^{2} \rho^{\prime}$. 
noise from the quadrupole source had to be added to the thickness noise calculations to fully account for high-speed impulsive noise. Efforts to calculate rotor noise in forward flight using noncompact source descriptions were initiated in both the U.S. and abroad. Most of this work was directed toward the prediction of discrete-frequency noise.

Even though computational work followed the theories in the seventies, computers were only beginning to have the necessary power for routine computations. This lack of computational power limited acoustic code development and is the origin of some of the approximations in current codes. Aerodynamic theories also were not sophisticated enough at this time to satisfy the input requirements of acoustic codes. This situation was beginning to change by the end of the decade, especially at NASA Ames which enjoyed the lead in supercomputing technology. The computing power available to $\mathrm{Yu}$, Caradonna and Schmitz $[17,19]$ is at least partially responsible for their demonstration of the importance of the quadrupole source in the $\mathrm{FW}-\mathrm{H}$ equation. The quadrupole was neglected in most theories because of the lack of information about the flow field around the rotor. George [20] and Schmitz and Yu [21] reviewed the status of helicopter noise in the late 1970's and the early 1980's, respectively.

By the 1980's, the emphasis on theoretical development combined with several model scale and flight tests had led to a fairly complete understanding and classification of the physical sources of helicopter rotor noise [21]. This did not immediately translate into an ability to predict these sources, however. The threat of noise certification and regulation forced the U.S. helicopter industry into the unfamiliar ${ }^{4}$ position of collaborating in their development of a "design for noise" capability. NASA [22] organized and led the cooperation between the four major U.S. manufacturers ${ }^{5}$ through the NASA/AHS Rotorcraft Noise Reduction Program, $(\mathrm{NR})^{2}$. The $(\mathrm{NR})^{2}$ program improved the U.S. prediction capability in several ways. ${ }^{6}$ Each of the four companies increased their acoustic staff and enhanced their acoustic facilities. The program also encouraged the participation of rotor aerodynamicists and fluid mechanicians in rotor acoustics. The increased emphasis by government and industry was well timed to build on the theoretical developments of the previous decade, hence prediction codes resulted from the effort.

In parallel with the $(\mathrm{NR})^{2}$ program were several high quality acoustic tests conducted in the newly opened German-Dutch wind tunnel (DNW ${ }^{7}$ ) [23-27].

\footnotetext{
$\overline{4}$ Unfamiliar because the rotorcraft industry had not seriously considered noise in rotorcraft design up to this point.

5 Bell Helicopter Textron, Inc., Boeing Helicopters, McDonnell Douglas Helicopter Company and Sikorsky Aircraft

6 This work also indicated that much more work was required to give the necessary accuracy for routine prediction.

7 Duits-Nederslandse Windtunnel.
} 
These tests greatly aided the prediction work by concentrating on individual source mechanisms and clarifying the understanding of the physical mechanisms. Advances due to the (NR) ${ }^{2}$ program, the DNW experimental efforts and increases in computer power, have greatly improved the current state of helicopter noise prediction.

\section{Theoretical Background: The Ffowcs Williams - Hawkings Equa- tion}

The problem of aerodynamically generated sound is governed by the conservation laws of mass, momentum and energy for a fluid. The FW-H equation [11] is an exact rearrangement of the continuity equation and the Navier-Stokes equations into the form of an inhomogeneous wave equation with two surface source terms and a volume source term. The FW-H equation is the most general form of the Lighthill acoustic analogy [12] because it extends the analogy to include general surfaces in arbitrary motion. The $\mathrm{FW}-\mathrm{H}$ equation is the appropriate tool for predicting the noise generated by the complex motion of helicopter rotors. Today almost all deterministic rotor noise predictions are based on time-domain integral formulations of the $\mathrm{FW}-\mathrm{H}$ equation.

In this section, the $\mathrm{FW}-\mathrm{H}$ equation will be examined to provide the background necessary to understand current rotor noise prediction models. First, the derivation of the partial differential equation form of the $\mathrm{FW}-\mathrm{H}$ equation is presented along with a brief explanation of the underlying mathematical theory of generalized functions. This is followed by an interpretation of the source

terms and a brief description of some of the important integral formulations of the $\mathrm{FW}-\mathrm{H}$ equation.

\subsection{Derivation}

The derivation of the FW-H equation follows the same steps as Lighthill used to derive his acoustic analogy for jet noise [12], yet is applicable to a wider range of problems because of its use of generalized function theory (i.e., theory of distributions). The fundamentals of generalized function theory will be presented, followed by the derivation of the $\mathrm{FW}-\mathrm{H}$ equation.

\subsubsection{Preliminaries}

The FW-H equation may be derived by embedding the exterior flow problem in unbounded space, i.e., by extending the definition of the fluid properties 
such that inside the moving surface the flow parameters have the same fluid state as the undisturbed medium. The flow parameters will then have artificially introduced discontinuities across the moving surface. Since we need the derivatives of these parameters in conservation laws, generalized function theory is the ideal tool for this problem. The fundamentals of this theory for application to aeroacoustics and aerodynamics are presented in two works by Farassat $[28,29]$. We highly recommend the books by Gel'fand and Shilov (Volume 1) [30], Jones (2nd edition) [31] and Kanwal [32] for the more advanced aspects of generalized function theory.

To learn and apply this theory requires considerable mathematical maturity. Nevertheless, we believe that a basic understanding of generalized differentiation will be sufficient to follow the derivation of the FW-H equation. We will give the formulas for generalized differentiation of discontinuous functions. Other important facts related to this subject that we use in this paper are:

(1) Conservation laws of mass continuity and momentum equation (written in conservative form known as the Reynolds form) are valid for fluid flows with real and artificial discontinuities if we interpret all derivatives as generalized derivatives.

(2) The Green's function approach can be used to find discontinuous solutions of differential equations provided that the problem is set up in the generalized function space.

We now give some results for generalized differentiation of discontinuous functions. Let $h(x)$ be a function that is piecewise smooth with a discontinuity at $x_{o}$ and a jump of $\Delta h=h\left(x_{o+}\right)-h\left(x_{o-}\right)$ at this point. We use a bar over the derivative symbol to denote generalized differentiation. Then the generalized derivative of $h(x)$ is defined as

$$
\frac{\bar{d} h}{d x}=\bar{h}^{\prime}(x)=h^{\prime}(x)+\Delta h \delta\left(x-x_{o}\right)
$$

where $h^{\prime}(x)$ is the ordinary derivative of $h(x)$. Here $\delta(x)$ is the Dirac delta function. One significant fact to remember about generalized differentiation is that for all $x$ such that $a<x_{o}<x$, we have:

$$
\int_{a}^{x} \frac{\bar{d} h}{d x} d x=h(x)-h(a) \neq \int_{a}^{x} h^{\prime}(x) d x .
$$

This means that $\bar{h}^{\prime}(x)$ retains the memory of the jump at $x_{o}$ but $h^{\prime}(x)$ does not. To illustrate this result, we consider the Heaviside function

$$
H(x)= \begin{cases}0 & x<0 \\ 1 & x>0\end{cases}
$$


We have $H^{\prime}(x)=0$ and $\Delta H=1$ at $x=0$, therefore,

$$
\bar{H}^{\prime}(x)=\delta(x)
$$

It is seen that for $a<0$ and any $x$

$$
\int_{a}^{x} \bar{H}^{\prime}(x) d x=\int_{a}^{x} \delta(x) d x=H(x)
$$

which is not the same as

$$
\int_{a}^{x} H^{\prime}(x) d x=0
$$

Equation (2) describes the extension of the differentiation process in generalized function theory. This fact is one of the main reasons for the success of this theory in working with discontinuous functions. In short, the machinery of generalized function theory makes it just as easy to work with discontinuous or nonsmooth functions as working with smooth functions in classical analysis.

Now let $q(\mathbf{x})$ be a function of $\mathbf{x}=\left(x_{1}, x_{2}, x_{3}\right)$ with a discontinuity across the surface $f(\mathbf{x})=0$. Let us define the jump $\Delta q$ of $q(\mathbf{x})$ across $f=0$ as follows:

$$
\Delta q=q(f=0+)-q(f=0-)
$$

Note that $\Delta q$ is a function of position on the surface of discontinuity $f=0$ and $\nabla f$ points into the region $f>0$. The generalized partial derivative of $q(\mathbf{x})$ with respect to the variable $x_{i}$ is

$$
\frac{\bar{\partial} q}{\partial x_{i}}=\frac{\partial q}{\partial x_{i}}+\Delta q \frac{\partial f}{\partial x_{i}} \delta(f)
$$

where $\delta(f)$ is the Dirac delta function. Here $\partial q / \partial x_{i}$ is the ordinary partial derivative of $q(\mathbf{x})$. From equation (9), the generalized gradient of $q(\mathbf{x})$ can be written as

$$
\bar{\nabla} q=\nabla q+\Delta q \nabla f \delta(f)
$$

(For the generalized divergence and curl of discontinuous vector functions see Farassat $[28,29]$.)

A useful result for the integration of $\delta(f)$ is the following:

$$
\int Q(\mathbf{x})|\nabla f| \delta(f) d \mathbf{x}=\int_{f=0} Q(\mathbf{x}) d S
$$

where $Q(\mathbf{x})$ is an arbitrary function. The integral on the right of equation (11) is a surface integral over the surface $f=0$ with $Q(\mathbf{x})$ evaluated on the surface. 
Another important fact to remember is that the order of operations can be exchanged when working with generalized functions:

$$
\begin{aligned}
\frac{\bar{\partial}^{2} Q(\mathbf{x})}{\partial x_{i} \partial x_{j}} & =\frac{\bar{\partial}^{2} Q(\mathbf{x})}{\partial x_{j} \partial x_{i}} \\
\frac{\bar{\partial}}{\partial x_{i}} \int Q(\mathbf{x}, \mathbf{y}) d \mathbf{y} & =\int \frac{\bar{\partial}}{\partial x_{i}}(Q(\mathbf{x}, \mathbf{y})) d \mathbf{y}
\end{aligned}
$$

where in equation (13) the limits of the integrals are not functions of the variable $x_{i}$. This property is one of the most useful and powerful properties of generalized functions. It is often used in applications without the need for justification as required in classical analysis, thus speeding up the process of solving mathematical problems.

It is the experience of the authors that with some effort, engineers and students can get a good working knowledge of generalized function theory without the need to learn all the highly abstract mathematical foundations. However, we give two observations here to help readers interested in learning more about generalized function theory:

(1) Although generalized function theory of one variable is very useful in applications such as signal analysis, in acoustics we need the theory for multidimensional spaces (usually 2, 3 or 4 dimensional spaces). Most books, however, do not emphasize multidimensional theory. The exceptions are Gel'fand and Shilov [30], Kanwal [32] and Jones [31]. In the multidimensional case, one also needs a working knowledge of differential geometry at the level of Struik [33] and general tensor analysis [34,35]. Farassat has included all of the necessary background theory and results for applications in aeroacoustics and aerodynamics in two NASA publications $[28,29]$.

(2) Generalized function theory has substantially extended the power of the classical analysis in solving mathematical problems, particularly those involving ordinary or partial differential equations. Often the solution is transparent when generalized function theory is used, while obtaining the solution using classical analysis is obscure or very difficult. For this reason, we remind the reader that generalized function theory is an indispensable tool for solving theoretical engineering problems.

\subsubsection{The Ffowcs Williams-Hawkings Equation}

Now we utilize generalized function theory to derive the FW-H equation. Let $f(\mathbf{x}, t)=0$ be a moving surface enclosing the noise generating region of the flow, possibly including solid surfaces such as rotor blades. We will refer to $f(\mathbf{x}, t)$ as a data surface for the reason that becomes clear below. We are 
interested in computing the sound field in the exterior of the data surface. We assume that $f>0$ in the exterior of the data surface and $f<0$ in the interior. Furthermore, to simplify algebraic manipulations, we assume that $f(\mathbf{x}, t)$ is defined such that $\nabla f=\hat{\mathbf{n}}$, the outward unit normal vector. If the original definition of $f$ does not satisfy this condition, then $f(\mathbf{x}, t)$ can always be redefined as $f /|\nabla f|$ which does satisfy this condition on the surface. We do not assume that the data surface is impenetrable because this will give us the most general result for applications. Our aim is to use the acoustic analogy to derive a wave equation with inhomogeneous sources in the volume exterior to and on the data surface itself. Here the generalized function theory simplifies the derivation enormously and provides a method completely parallel to Lighthill's derivation of the jet noise equation [12].

We assume that the fluid extends inside the data surface with the conditions of the undisturbed quiescent medium, which, with the exception of the sound speed $c$, we denote with the subscript $o$. The reason for this extension (i.e., embedding the original problem into unbounded space) is that we want our wave equation to be valid in the entire three-dimensional space so that we can use the simple Green's function of the wave equation in unbounded space. We have now introduced an artificial discontinuity in all fluid parameters across the data surface $f=0$. Therefore, to use the conservation laws, we assume all flow parameters are generalized functions and we work with conservation laws with generalized derivatives. For now we assume that the flow parameters have no discontinuities other than those across the data surface. The general situation will be discussed later. The mass continuity and conservation of momentum equations within the fluid (i.e., in the region $f>0$ ) are:

$$
\begin{gathered}
\frac{\partial \rho}{\partial t}+\frac{\partial}{\partial x_{i}}\left(\rho u_{i}\right)=0 \\
\frac{\partial}{\partial t}\left(\rho u_{i}\right)+\frac{\partial}{\partial x_{j}}\left(\rho u_{i} u_{j}+P_{i j}\right)=0
\end{gathered}
$$

where $\rho$ is the fluid density, $\rho u_{i}$ is the component of fluid momentum and $P_{i j}$ is the compressive stress tensor as defined by Lighthill [12]. The momentum equation (15) is written in the conservative form, sometimes known as the Reynolds form, to avoid the problem of multiplication of generalized functions. $^{8}$

We know that within the fluid the conservation laws hold with both ordinary and generalized derivatives. Let us now use the rules of generalized differentiation to see how these conservation laws change when the artificial discontinuity

8 Multiplication of generalized functions is an advanced subject that will not be encountered in our work, therefore we will not discuss it here. We interpret the vector components $\rho u_{i}$ and tensor components $\rho u_{i} u_{j}$ as unique generalized functions as opposed to the product of separate generalized functions. 
we introduced across $f=0$ is included and the ordinary derivatives are replaced with generalized derivatives. In the case of the continuity equation we have

$$
\begin{aligned}
\frac{\bar{\partial} \rho}{\partial t}+\frac{\bar{\partial}}{\partial x_{i}}\left(\rho u_{i}\right) & =\frac{\partial \rho}{\partial t}+\left(\rho-\rho_{o}\right) \frac{\partial f}{\partial t} \delta(f)+\frac{\partial}{\partial x_{i}}\left(\rho u_{i}\right)+\left(\rho u_{i}\right) \frac{\partial f}{\partial x_{i}} \delta(f) \\
& =\left[\rho_{o} v_{n}+\rho\left(u_{n}-v_{n}\right)\right] \delta(f)
\end{aligned}
$$

where $u_{n}=u_{i} n_{i}$ is the local fluid velocity in the direction normal to the data surface, $n_{i}=\partial f / \partial x_{i}$ is the component of the unit outward normal to $f=0$ and $v_{n}=-\partial f / \partial t$ is the local normal velocity of the data surface. Here $\left(\rho-\rho_{o}\right)$ is the jump in $\rho$ at $f=0$ and $\rho u_{i}$ is the jump in $\rho u_{i}$ at $f=0$. Note that we have used equation (14) on the right of the first equality to find the second equality. The above result indicates that the artificial discontinuity at $f=0$ has introduced a source term on the right side of the mass continuity equation that is proportional to the local rate of mass injection into the exterior domain. The analytical form of the expression for the mass injection rate has been readily obtained from the rules of generalized differentiation!

Similarly, source terms are introduced by the artificial discontinuity across the data surface in the momentum equation. The generalized momentum equation is then

$$
\begin{aligned}
\frac{\bar{\partial}}{\partial t}\left(\rho u_{i}\right)+\frac{\bar{\partial}}{\partial x_{j}}\left(\rho u_{i} u_{j}+P_{i j}\right) & =\frac{\partial}{\partial t}\left(\rho u_{i}\right)+\rho u_{i} \frac{\partial f}{\partial t} \delta(f) \\
& +\frac{\partial}{\partial x_{j}}\left(\rho u_{i} u_{j}+P_{i j}\right)+\left(\rho u_{i} u_{j}+\Delta P_{i j}\right) \frac{\partial f}{\partial x_{j}} \delta(f) \\
& =\left[\rho u_{i}\left(u_{n}-v_{n}\right)+\Delta P_{i j} n_{j}\right] \delta(f) .
\end{aligned}
$$

We have used equation (15) on the right side of the first equality in equation (17) to simplify the second equality. We see on the right side of the momentum equation with generalized derivatives that the artificial discontinuity across the data surface has introduced a source proportional to the sum of the local force intensity $\Delta P_{i j} n_{j}$ on $f=0$ and the rate of momentum injection, $\rho u_{i}\left(u_{n}-v_{n}\right)$, into the exterior of $f=0$. We remind the reader that all fluid parameters in equations (16) and (17) refer to the exterior side of the data surface.

The next step in the derivation of the FW-H equation follows exactly Lighthill's derivation of the jet noise equation except that all new derivatives taken are generalized derivatives. First take $\bar{\partial} / \partial t$ of both sides of equation (16), then $\bar{\partial} / \partial x_{i}$ of both sides of equation (17) and subtract the later from the former. Finally subtract $\bar{\nabla}^{2}\left[c^{2}\left(\rho-\rho_{o}\right)\right]$ from both sides of the equation and rearrange 
to keep the wave operator acting on $c^{2}\left(\rho-\rho_{o}\right)$ on the left side of the equation. The resulting equation is the Ffowcs Williams-Hawkings equation for a penetrable or permeable data surface. Using $p^{\prime}$ for $c^{2}\left(\rho-\rho_{0}\right)$ on the left side ${ }^{9}$, this equation is:

$$
\begin{aligned}
\bar{\square}^{2} p^{\prime}(\mathbf{x}, t)= & \frac{\partial}{\partial t}\left\{\left[\rho_{o} v_{n}+\rho\left(u_{n}-v_{n}\right)\right] \delta(f)\right\} \\
& -\frac{\partial}{\partial x_{i}}\left\{\left[\Delta P_{i j} \hat{n}_{j}+\rho u_{i}\left(u_{n}-v_{n}\right)\right] \delta(f)\right\} \\
& +\frac{\bar{\partial}^{2}}{\partial x_{i} \partial x_{j}}\left[T_{i j} H(f)\right]
\end{aligned}
$$

where $T_{i j}=\rho u_{i} u_{j}+P_{i j}-c^{2} \rho^{\prime}$ is the Lighthill stress tensor $\left(\rho^{\prime}=\rho-\rho_{o}\right)$ and on the left side we use the notation $\bar{\square}^{2}=\left[\left(1 / c^{2}\right)\left(\bar{\partial}^{2} / \partial t^{2}\right)\right]-\bar{\nabla}^{2}$. Note that we have not used a bar over $\partial / \partial t$ and $\partial / \partial x_{i}$ in the first two terms on the right side of equation (18) because it is obvious that these can only be generalized differentiation - due to the presence of $\delta(f)$ in the expression inside the curly brackets. The Heaviside function $H(f)$ in the last term on the right of equation (18) is used here, following Ffowcs Williams and Hawkings, to remind the reader that this volume source only exists exterior to the data surface. By assumption $T_{i j}=0$ inside the data surface. Note that, in general, the viscous term in $\Delta P_{i j}$ is a negligible source sound and one can often assume that $\Delta P_{i j}=\left(p-p_{o}\right) \delta_{i j}$, where $\delta_{i j}$ is the Kronecker delta. Ffowcs Williams and Hawkings chose to write $p$ instead of $p-p_{o}$ in the second source term of the FW-H equation - they then regarded $p$ as the gauge pressure in that source term. This has caused some confusion among users which we will rectify here by defining $\Delta P_{i j}=\left(p-p_{o}\right) \delta_{i j}$ when the fluid is inviscid.

\subsection{Interpretation and Observations}

The first two source terms in equation (18) are monopole (thickness) and dipole (loading) sources, respectively, based on their mathematical structure. Both of these sources are surface sources: i.e., they act only on the surface $f=0$ as indicated by the presence of the Dirac delta function $\delta(f)$. The reader is warned, however, that when the data surface is in motion, these sources behave differently than stationary monopole and dipole sources and care must be exercised when making physical arguments. The third source term is a quadrupole source term that acts throughout the volume that is exterior to the data surface - as indicated by the Heaviside function $H(f)$.

${ }_{9}$ Outside the source region we expect linear wave propagation, hence $p^{\prime}=c^{2} \rho^{\prime}$ is valid in this region. 
The three source terms on the right of equation (18) have become known as the thickness, loading and quadrupole source terms, respectively, although this terminology is appropriate only if the data surface corresponds to a solid (impermeable) surface.

If the data surface $f=0$ coincides with a solid surface, then the normal velocity of the fluid is the same as the normal velocity of the surface $\left(u_{n}=v_{n}\right)$. In this case equation (18) can be written

$$
\bar{\square}^{2} p^{\prime}(\mathbf{x}, t)=\frac{\partial}{\partial t}\left\{\left[\rho_{o} v_{n}\right] \delta(f)\right\}-\frac{\partial}{\partial x_{i}}\left\{\left[\Delta P_{i j} \hat{n}_{j}\right] \delta(f)\right\}+\frac{\bar{\partial}^{2}}{\partial x_{i} \partial x_{j}}\left[T_{i j} H(f)\right]
$$

which is the most common partial differential form of the $\mathrm{FW}-\mathrm{H}$ equation. The monopole or thickness source term in equation (19) models the noise generated by the displacement of fluid as the body passes (i.e., thickness noise). The dipole or loading source term in equation (19) models the noise that results from the unsteady motion of the force distribution on the body surface (i.e., loading noise, blade-vortex-interaction noise and even broadband noise). The thickness and loading source terms have been used for several years in rotor noise prediction because they account for most of the acoustic signal when the flow field is not transonic or supersonic. Furthermore, they do not require knowledge of the flow field off the blade (although the accurate determination of the blade-surface pressure is still challenging).

The quadrupole source term in equation (19) models the nonlinearities due to both the local sound speed variation and the finite fluid velocity near the blade. The quadrupole source term is required to accurately predict both the waveform shape and amplitude of high-speed impulsive noise. The importance of the quadrupole term has long been recognized [18, 19], however, the quadrupole source has often been neglected in rotor noise prediction because of the computational demands of determining the flow field with sufficient accuracy to be used for noise prediction and the computational challenge of volume integration in the acoustic prediction. If equation (18) is used with a permeable data surface that encloses the acoustic sources, then the surface source terms in equation (18) also account for the sound generated by acoustic sources in the flow field outside of the blade surface but inside the data surface. In this case the volume source in equation (18) may have a negligible contribution. This extremely valuable observation will be discussed further in section 6.4 .

It is interesting to note that the form of the various source terms is not unique. One example of this is known as Isom thickness noise [36]. This can be demonstrated by using the example given by Ffowcs Williams (and reported by Farassat [37]). First, consider the generalized differentiation of the trivial function 
$[1-H(f)]$ with respect to time which is

$$
\frac{\partial}{\partial t}[1-H(f)]=-\frac{\partial f}{\partial t} \delta(f)=v_{n} \delta(f) .
$$

Recall that $H(f)$ is the Heaviside function, hence $H(f)=1$ outside the surface $f=0$ and the left side of equation (20) is zero in this region. Also recall that $v_{n}$ is the local normal velocity of the surface and the function $f$ has been defined such that $|\nabla f|=1$. Taking another time derivative of both sides of equation (20) gives the following result:

$$
\frac{\partial^{2}}{\partial t^{2}}[1-H(f)]=\frac{\partial}{\partial t}\left[v_{n} \delta(f)\right]
$$

In a similar fashion, the Laplacian operator can be applied to the function $[1-H(f)]$ yielding:

$$
\nabla^{2}[1-H(f)]=-\frac{\partial}{\partial x_{i}}\left[\hat{n}_{i} \delta(f)\right]
$$

Using equation (21) and equation (22), the following wave equation is obtained:

$$
\left[\frac{1}{c^{2}} \frac{\partial^{2}}{\partial t^{2}}-\nabla^{2}\right]\left\{\rho_{o} c^{2}[1-H(f)]\right\}=\frac{\partial}{\partial t}\left[\rho_{o} v_{n} \delta(f)\right]+\frac{\partial}{\partial x_{i}}\left[\rho_{o} c^{2} \hat{n}_{i} \delta(f)\right] .
$$

Because the trivial function $\rho_{o} c^{2}[1-H(f)]$ is always zero outside the surface $f=0$, we see that

$$
\frac{\partial}{\partial t}\left[\rho_{o} v_{n} \delta(f)\right]=-\frac{\partial}{\partial x_{i}}\left[\rho_{o} c^{2} \hat{n}_{i} \delta(f)\right]
$$

where the term on the left side of equation (24) is recognized as the thickness source term in equation (19) and the term on the right side of equation (24) is the loading source term of equation (19) with with a constant pressure jump $p-p_{o}=\rho_{o} c^{2}$. Although mathematically equivalent, the two formulations for thickness noise have quite different characteristics and robustness when integrated numerically [38]. More examples of the nonuniqueness of the source description are given by Ffowcs Williams [39] and Kanwal [32].

We now return to the question of how the effect of other flow discontinuities on noise generation can be studied using the $\mathrm{FW}-\mathrm{H}$ equation and whether equation (18) should be modified for this purpose. Shocks, thin wakes and vortices are flow discontinuities that can be potential sources of sound from the point of view of the acoustic analogy. A careful examination of our derivation of equation (18) shows that discontinuities in the flow field will not modify the $\mathrm{FW}-\mathrm{H}$ equation. However, care must be exercised in taking the generalized space derivatives of $T_{i j}$ because any flow discontinuity, such as a shock, will introduce surface sources on the discontinuity surface [40,41]. 
Let's look back and see what we have achieved. The FW-H equation is the governing equation for computation of the noise outside of the surface $f=0$. It requires knowledge of $\rho, \rho u_{i}$ and $p$ on the data surface and $T_{i j}$ outside of this surface. These quantities are usually obtained from a CFD computation and, in general, their computation is a difficult task. This difficulty has made some researchers dubious about the usefulness of the acoustic analogy approach and has led them to search for alternative methods such as finite difference schemes. The argument here is that if one has to use substantial computer resources to supply the data needed as input to the $\mathrm{FW}-\mathrm{H}$ equation, then perhaps propagation in the typically linear region external to the data surface can be computed for only a little extra effort. Such an argument has several weaknesses, which we will point out. First, the FW-H equation is linear and many different closed form solutions can be derived and efficiently coded for numerical work. Furthermore, such closed form solutions can be used as the basis of qualitative arguments for noise control. Second, most numerical schemes have dispersion and dissipation errors that depend on the spatial and temporal grid resolution. Because of the broad frequency range of interest in acoustics, most purely numerical schemes cannot compete in accuracy and efficiency with codes based on the $\mathrm{FW}-\mathrm{H}$ equation. We foresee that this situation will not change for a long time. Finally, a much larger computational domain is needed for an acoustic problem than for an aerodynamic problem. This increased domain size increases computational resource requirements dramatically. Therefore, the acoustic analogy approach is an ideal partner to CFD: the near-field should be computed with unsteady CFD methods while the $\mathrm{FW}-\mathrm{H}$ equation is appropriate for the prediction of the acoustic propagation.

\subsection{Integral Formulations}

To realize the advantages of the $\mathrm{FW}-\mathrm{H}$ equation as compared to CFD methods, an integral formulation of the $\mathrm{FW}-\mathrm{H}$ equation should be used. The key feature of the $\mathrm{FW}-\mathrm{H}$ equation is that it is an inhomogeneous wave equation for the external flow problem that has been embedded in unbounded space. Hence, an integral representation of the solution can be readily found using the free-space Green's function $(\delta(g) / 4 \pi r$, with $g=\tau-t+r / c)$. We use the term "integral representation" because the integral formulation must either be solved as a singular integral equation or, as is most often done in rotor noise prediction, the flow-field data in the source region must be given as input to determine the acoustic solution outside the source region. The particular for-

mulation that is developed results primarily from the choice of the change of variables needed to analytically integrate the Dirac delta functions.

To illustrate the above explanation, consider the following example. An inho- 
mogeneous wave equation can be written as

$$
\square^{2} \phi(\mathbf{x}, t)=Q(\mathbf{x}, t) \delta(f)
$$

where $\square^{2}$ is the wave operator, $Q(\mathbf{x}, t)$ is the source strength and $f(\mathbf{x}, t)=0$ defines a surface over which the source is distributed. Equation (25) is typical of the various source terms in the FW-H equation (either (18) or (19)). By using the free-space Green's function $\delta(g) / 4 \pi r$, an integral representation of the solution may be written as

$$
4 \pi \phi(\mathbf{x}, t)=\int_{-\infty}^{t} \int_{-\infty}^{\infty} \frac{Q(\mathbf{y}, \tau) \delta(f) \delta(g)}{r} d \mathbf{y} d \tau
$$

The next stage in the development of the acoustic formulation is to integrate the Dirac delta functions $\delta(f)$ and $\delta(g)$, a process that requires a change of variables. The change of variables determines the type of formulation. Equation (26) can be expressed as

$$
\begin{aligned}
4 \pi \phi(\mathbf{x}, t) & =\int_{f=0}\left[\frac{Q(\mathbf{y}, \tau)}{r\left|1-M_{r}\right|}\right]_{r e t} d S \\
& =\int_{-\infty}^{t} \int_{\substack{f=0 \\
g=0}} \frac{Q(\mathbf{y}, \tau)}{r \sin \theta} c d \Gamma d \tau \\
& =\int_{F=0} \frac{1}{r}\left[\frac{Q(\mathbf{y}, \tau)}{\Lambda}\right]_{r e t} d \Sigma
\end{aligned}
$$

with the variable transformations $\left(y_{3}, \tau\right) \rightarrow(f, g),\left(y_{2}, y_{3}\right) \rightarrow(f, g)$ and $\left(y_{3}, \tau\right) \rightarrow$ $(F, g)$, respectively. Equation $(27)$ is obtained by noting that

$$
d \mathbf{y} d \tau=\frac{d y_{1} d y_{2} d f d g}{\left|\partial f / \partial y_{3}\right|\left|1-M_{r}\right|}=\frac{d S d f d g}{\left|1-M_{r}\right|} ;
$$

equation (28) is obtained by noting that

$$
d \mathbf{y}=\frac{d y_{1} d f d g}{\partial(f, g) / \partial\left(y_{2}, y_{3}\right)}=\frac{d y_{1} d f d g}{(\nabla f \times \nabla g) \cdot \hat{e}_{1}}=\frac{c d \Gamma d f d g}{\sin \theta}
$$

and equation (29) is obtained by noting that

$$
d \mathbf{y} d \tau=\frac{d y_{1} d y_{2} d F d g}{\partial(F, g) / \partial\left(y_{3}, \tau\right)}=\frac{d y_{1} d y_{2} d F d g}{\partial F / \partial y_{3}}=\frac{d y_{1} d y_{2} d F d g}{N_{3}|\nabla F|}=\frac{d \Sigma d F d g}{|\nabla F|}
$$

where the function $F(\mathbf{y} ; \mathbf{x}, t)=f(\mathbf{y}, t-r / c),|\nabla F| \equiv \Lambda$, and $N_{3}$ is the third component of the unit normal vector to the surface $F=0$. The geometrical 
proof of

$$
\frac{d S}{\left|1-M_{r}\right|}=\frac{c d \tau d \Gamma}{\sin \theta}=\frac{d \Sigma}{\Lambda}
$$

was given in ref. 14. More recently a completely analytical derivation was given by Farassat and Farris [42].

The three formulations expressed in equations (27)-(29) are termed the retardedtime, collapsing-sphere and emission-surface formulations, respectively. Timedomain-acoustic formulations can be classified as one of these generic types; each type has its own physical and geometrical interpretation. These formulations and their interpretations will be discussed in more detail in section 5 . If the source in equation (25) had been a volume distribution, analogous integral formulas would result. (Ref. 16 discusses many of the specific linear formulations used in rotating blade noise prediction.)

Nearly all rotor noise prediction codes have used one of two formulations developed by Farassat. In the following subsections, some of the important details of their development are presented.

\subsubsection{Formulation 1}

Although similar to the previous example, the thickness and loading source terms in the FW-H equation (either equation (18) or (19)) have the additional complexity of time and space differentiation of the source terms, including the Dirac delta function $\delta(f)$. One approach to dealing with this complexity is to recognize that the wave operator $\square^{2}$ is a linear operator, hence both the temporal and spatial derivatives commute. Thus the formal solution of the FW-H equation (19) can be written

$$
4 \pi p^{\prime}(\mathbf{x}, t)=\frac{\partial}{\partial t} \int_{-\infty}^{t} \int_{-\infty}^{\infty} \frac{\rho_{o} v_{n} \delta(f) \delta(g)}{r} d \mathbf{y} d \tau-\frac{\partial}{\partial x_{i}} \int_{-\infty}^{t} \int_{-\infty}^{\infty} \frac{\ell_{i} \delta(f) \delta(g)}{r} d \mathbf{y} d \tau
$$

where $\ell_{i}=\Delta P_{i j} \hat{n}_{j}$ is the local force acting on the impenetrable data surface and the quadrupole source has been neglected. Next, the divergence operator can be taken inside the second integral where it acts on $\delta(g) / r$. If we recall that $g=\tau-t+r / c$ and note that $\partial r / \partial x_{i}=\hat{r}_{i}$, then

$$
\frac{\partial}{\partial x_{i}}\left(\frac{\delta(g)}{r}\right)=-\frac{\delta^{\prime}(g)}{c r} \hat{r}_{i}-\frac{\delta(g)}{r} \hat{r}_{i}
$$

Notice also that

$$
\frac{\partial}{\partial t}\left(\frac{\delta(g)}{r}\right)=-\frac{\delta^{\prime}(g)}{r}
$$


which can be used to eliminate the $\delta^{\prime}(g)$ in equation (35). Using equations (35) and (36) to eliminate the divergence operator in equation (34) yields:

$$
4 \pi p^{\prime}(\mathbf{x}, t)=\frac{1}{c} \frac{\partial}{\partial t} \int_{-\infty}^{t} \int_{-\infty}^{\infty} \frac{\left(\rho_{o} c v_{n}+\ell_{r}\right) \delta(f) \delta(g)}{r} d \mathbf{y} d \tau+\int_{-\infty}^{t} \int_{-\infty}^{\infty} \frac{\ell_{r} \delta(f) \delta(g)}{r^{2}} d \mathbf{y} d \tau
$$

and a change of variables $\left(\tau, y_{3}\right) \rightarrow(g, f)$ results in the retarded-time formulation Farassat has called Formulation 1:

$$
4 \pi p^{\prime}(\mathbf{x}, t)=\frac{1}{c} \frac{\partial}{\partial t} \int_{f=0}\left[\frac{\rho_{o} c v_{n}+\ell_{r}}{r\left|1-M_{r}\right|}\right]_{r e t} d S+\int_{f=0}\left[\frac{\ell_{r}}{r^{2}\left|1-M_{r}\right|}\right]_{r e t} d S .
$$

In equation (37) the subscript ret implies that the integrand is evaluated at the retarded time (i.e., the time when the sound is emitted) and the integration is performed over the actual blade surface $f=0$. This formulation has the advantage that spatial differentiation - which would need to be done numerically - is avoided.

One drawback of Formulation 1 is that the time derivative of the first integral must still be performed. The FW-H equation is applicable only for a stationary observer, yet the acoustic pressure computation for each observer time is independent of all other observer times. Therefore, the observer position can be moved for each point in the acoustic pressure time history computation to provide the acoustic solution for the case of a moving observer (e.g., a wind tunnel test or the case of a microphone mounted on the helicopter). In this situation, the integral must be evaluated at the same observer position for each time used to numerically compute the time derivative of the integral.

\subsubsection{Formulation $1 A$}

The speed and accuracy of the noise calculation is improved by elimination of the time derivative of the first integral in Formulation 1. Using the definition of the retarded time function $g$ and the fact that $r$ is a function of $\tau$ gives

$$
\left.\frac{\partial}{\partial t}\right|_{\mathbf{x}}=\left(\left.\frac{1}{1-M_{r}} \frac{\partial}{\partial \tau}\right|_{\mathbf{x}}\right)_{r e t}
$$

where the $\left.\right|_{\mathbf{x}}$ implies that the observer position $\mathbf{x}$ is fixed during the differentiation. This relation enables the time derivative to be taken inside the first integral of equation (37). When this is done the final result is

$$
\left.p^{\prime} \mathbf{x}, t\right)=p_{T}^{\prime}(\mathbf{x}, t)+p_{L}^{\prime}(\mathbf{x}, t)
$$

where 


$$
\begin{aligned}
4 \pi p_{T}^{\prime}(\mathbf{x}, t) & =\int_{f=0}\left[\frac{\rho_{o}\left(\dot{v}_{n}+v_{\dot{n}}\right)}{r\left|1-M_{r}\right|^{2}}\right]_{r e t} d S \\
& +\int_{f=0}\left[\frac{\rho_{o} v_{n}\left(r \dot{M}_{r}+c M_{r}-c M^{2}\right)}{r^{2}\left|1-M_{r}\right|^{3}}\right]_{r e t} d S \\
4 \pi p_{L}^{\prime}(\mathbf{x}, t) & =\frac{1}{c} \int_{f=0}\left[\frac{\dot{\ell}_{r}}{r\left|1-M_{r}\right|^{2}}\right]_{r e t} d S+\int_{f=0}\left[\frac{\ell_{r}-\ell_{M}}{r\left|1-M_{r}\right|^{2}}\right]_{r e t} d S \\
& +\frac{1}{c} \int_{f=0}\left[\frac{\ell_{r}\left(r \dot{M}_{r}+c M_{r}-c M^{2}\right)}{r^{2}\left|1-M_{r}\right|^{3}}\right]_{r e t} d S
\end{aligned}
$$

which is known as Formulation 1A. In these equations a dot over a variable implies the source time derivative of that variable and the subscript $n, r$ and $M$ refer to the dot product with the unit normal vector, the unit radiation vector, or the surface velocity vector normalized by the speed of sound, respectively. In this formulation, integrands with $1 / r$ dependence are far-field terms and those with $1 / r^{2}$ dependence are near-field terms. Although the integrands in Formulation 1A are somewhat more complicated than in Formulation 1, a numerical differentiation of an integral is not required. This is especially important for cases where the observer is moving. More details on the derivation of Formulation 1A can be found in refs. 43, 44 .

\section{Numerical Algorithms for Rotor Noise Prediction}

Most publications in rotor noise prediction focus on the analytical development and derivation of the formulation with only passing reference to its numerical implementation. While the formulation provides the framework and geometrical interpretation; we use the term "algorithm" to refer to how the integral is evaluated numerically. The accuracy, efficiency and robustness of the numerical algorithm depend strongly on the choice of both the formulation and the algorithm, nevertheless the details of the numerical implementation itself will affect code performance significantly. For example, high-order integration and adaptive quadrature algorithms can potentially be much more accurate and efficient than simpler counterparts for the same formulation. A discussion of some of the most widely used numerical algorithms - classified by formulation - is presented in this section. Although this discussion is primarily directed towards algorithms for surface-source distributions, analogous techniques are available for the integration of volume-source distributions. 


\subsection{Retarded-Time Algorithms}

We will first consider a generic retarded-time formulation

$$
4 \pi \phi(\mathbf{x}, t)=\int_{f=0}\left[\frac{Q(\mathbf{y}, \tau)}{r\left|1-M_{r}\right|}\right]_{r e t} d S
$$

where $Q$ is the known source strength, which is a function of the source position and time $(\mathbf{y}, \tau)$. Normally in acoustic predictions, $\phi$ is the acoustic pressure $p^{\prime}$. The interpretation of equation (42) is that integration occurs over the original surface $f=0$, with the integrand evaluated at the emission, or retarded, time $\tau^{*}=t-r / c$. The integration is easy to understand because the integration surface is given as part of the source description. Note that the formulation requires that the observer location $\mathbf{x}$ and the observer time $t$ are fixed during the evaluation of the integral. Numerical implementations of this formulation have proven to be very robust and efficient; hence, most acoustic-analogybased rotor noise predictions (e.g., refs. 44,45) and Kirchhoff codes (e.g., refs. $46,47)$ utilize retarded-time formulations.

\subsubsection{Mid-Panel Quadrature}

The most common method of numerically evaluating retarded-time integrals is to approximate the integral as follows:

$$
4 \pi \phi(\mathbf{x}, t) \approx \sum_{i=1}^{N}\left[\frac{Q\left(\mathbf{y}_{i}, t-r_{i} / c\right)}{r_{i}\left|1-M_{r}\right|_{i}}\right]_{r e t} \Delta S_{i}
$$

Here, the surface $S$ is divided into $N$ panels and the integrand is evaluated at the center of each panel $\left(\mathbf{y}_{i}\right)$ at that point's retarded time. ${ }^{10}$ If the source is not moving, then the determination of the retarded time $\tau^{*}$ is made by a simple computation $\tau^{*}=t-r_{i} / c$, where $r_{i}=\left|\mathbf{x}-\mathbf{y}_{i}\right|$. If the source is in motion, then the source position is a function of the retarded time (i.e., the desired $\mathbf{y}_{i}$ is $\mathbf{y}_{i}\left(\tau^{*}\right)$ ) and, unless the source motion is very simple, it is not practical to determine the retarded time analytically. The retarded time, then, is found numerically as the root of the equation $t-\tau-r(\tau) / c=0$. Even when the source motion is complex, such as for a helicopter main rotor, standard root-finding algorithms work well (e.g., see ref. 48). Because the source strength $Q$ is evaluated at the retarded time, temporal interpolation of the input data is usually required. A time history of $\phi$ is developed by choosing both the observer position and the observer time, evaluating the summation in equation (43) and then choosing the next observer time in the time history.

\footnotetext{
${ }^{10}$ Note that the subscript $i$ used in this section does not refer to the component of a vector, but rather is an index to the ith panel.
} 
Equations (42) and (43) can be used to find the time history of $\phi$ for a moving observer if the observer position is moved for each evaluation of the integral.

The approximation given in equation (43) with $\mathbf{y}_{i}$ at panel centers is in widespread use and works well as long as the panel size is sufficiently small. In this case, sufficiently small means that the source strength variation is approximately linear over the panel and that the retarded time does not vary significantly over the panel (i.e., $\Delta \tau<<$ typical period of fluctuations of $Q$ ). Refinement of the panel size is clearly needed if the source strength $Q$ is not linear over the panel because $Q$ is not resolved and the midpoint value is unlikely to represent the mean value. Insufficient resolution of the source strength can occur in practice whenever the noise source is physically localized, such as occurs in blade-vortex interaction noise for a helicopter rotors; therefore, we must require that the input data resolve the source spatially and temporally.

A subtle but extremely important point is that the variation of $Q$ over a panel in retarded-time space can be much more significant than the variation of $Q$ over a panel at single source time. Meadows and Atkins [49] noticed this phenomenon in a Kirchhoff computation of noise generated by an oscillating sphere. In their work, they used high-order interpolation and quadrature but found that more points per wavelength than expected were needed on the Kirchhoff surface to achieve the desired accuracy. Figure 3 (from ref. [49]) compares the pressure on the integration surface at one source time $\tau$ to the pressure as a function of retarded time $\tau^{*}$. Although the pressure plotted in figure 3 is not over one panel but over one meridian line on the spherical Kirchhoff surface, the idea is the same - the behavior of function in retardedtime space can be significantly different from that at any individual source time. A large panel size, certain observer orientations $(\hat{\mathbf{n}} \cdot \hat{\mathbf{r}} \rightarrow 0)$ and a large panel velocity $\left(M_{r} \rightarrow 1\right)$ can all lead to large variations in retarded time over a panel.

\subsubsection{High-Accuracy Quadrature}

A refinement of equation (43) can be made by replacing the single evaluation of the integrand at the panel center with more points; thus, greater accuracy is achieved. The discrete computation would then be

$$
4 \pi \phi(\mathbf{x}, t) \approx \sum_{i=1}^{N}\left(\sum_{j=1}^{n_{i}} \alpha_{j}\left[\frac{Q\left(\mathbf{y}_{j}, t-r_{j} / c\right)}{r_{j}\left|1-M_{r}\right|_{j}}\right]_{r e t}|J|_{j}\right) \Delta S_{i}
$$

where $\alpha_{j}$ and $|J|_{j}$ are the quadrature weight coefficient and determinant of the Jacobian of the transformation, respectively, for the $j$ th point in the panel quadrature algorithm. The weights $\alpha_{j}$ and the location of the quadrature points $\mathbf{y}_{j}$ can be chosen to increase the order of polynomial approximation used for the panel quadrature. Thus, with a larger number of points the limitations 
of the mid-panel algorithm can be overcome. Farassat et al. [50] and Dunn and Tarkenton [51] use a high-order quadrature algorithm of this type in their propeller noise prediction code ASSPIN.

The full benefit of a high-accuracy quadrature is only realized when a solutionadaptive quadrature scheme is used. This feature is included in equation (44) by selecting the number of quadrature points $n$ used in the $i$ th panel with some parameter related to the solution; that is, a larger number of quadrature points are used only when the function variation over the panel requires it. An adaptive-quadrature scheme can provide high accuracy and minimize the computational effort. Brentner and Holland [52] developed an adaptive-quadrature algorithm in a far-field quadrupole noise prediction. Their adaptation parameter was

$$
n_{i}\left(M_{r}, \Delta S_{i}\right) \propto \frac{\Delta S_{i}}{\left|1-M_{r}\right|}
$$

where $M_{r}$ (the Mach number of the panel center in the radiation direction) and $\Delta S_{i}$ (the physical area of the panel) were used to determine the number of Gauss-Legendre quadrature points in the chordwise direction of the panel; $n_{i}$ is scaled such that $1 \leq n_{i} \leq 20$. As $M_{r}$ approaches unity or if the area of the panel is large, the adaptation parameter becomes large. Similarly, if $M_{r}$ is small or the area of the panel is small, then the adaptation parameter becomes small. The computation with adaptive quadrature required little additional computer time but yielded a significantly better signal, as shown in figure 4 . (Note: The two remaining bumps at time $t=0.4$ and $t=1.5$ are an artifact of the chordwise truncation of the quadrupole source integration region ahead and behind the blade.) Although the adaptive quadrature of Brentner and Holland [52] worked well in their application, the ideal solution is an adaptive quadrature scheme that is based on a measure of error in the solution.

\subsubsection{Source-Time-Dominant Algorithm}

A completely different approach can be taken to evaluate a retarded-time integral if the source time is regarded as the primary time (i.e., dominant). Rather than select the observer time in advance, one can choose the source time for a panel (by again using the panel center) and determine when the signal will reach the observer. If the observer $\mathbf{x}$ is stationary, then $t=\tau+r_{i} / c$, which can be computed immediately. Otherwise, we must find the root of the equation $t-\tau-\left|\mathbf{x}(t)-\mathbf{y}_{i}(\tau)\right| / c=0$. The determination of $t$ even for the latter case is easier than finding the retarded time because observer motion is usually quite simple; hence, the solution for $t$ may be found analytically rather than by iteration. A sequence of source times (i.e., the times at which the source strength is available) will lead to a sequence of unequally spaced observer times. This panel time history can be interpolated to provide the contribution at the desired observer times. Interpolation in time is necessary 
so that the contributions from all source panels can be added together at the same observer times. This algorithm can be written symbolically as

$$
4 \pi \phi\left(\mathbf{x}, t^{*}\right) \approx \sum_{i=1}^{N} I\left(K_{i}(t), t^{*}\right)
$$

where $I\left(\cdot, t^{*}\right)$ is an interpolation operator and $t^{*}$ is the desired observer time. The approximation of the integral over the panel $K$ is defined as

$$
K_{i}(t)=\frac{Q\left(\mathbf{y}_{i}, \tau\right)}{r_{i}\left|1-M_{r}\right|_{i}} \Delta S_{i}
$$

The value of $t^{*}$ is determined by the selection of $\mathbf{y}_{i}$ and $\tau$. This algorithm has the advantage that a retarded-time calculation is not necessary per se and the discrete time-dependent input data do not need to interpolated. This characteristic is useful when a CFD code provides the input data. Another computational advantage of the source-time-dominant algorithm is that the solution process is inherently parallel; thus, the algorithm is a good candidate for parallel computers. Xue has implemented this algorithm in a general Kirchhoff aeroacoustics code [53] and Leishman [54] presented a version of this approach (which he called a "binning technique" 11 ) for rotor noise prediction. Recently, Brentner et al. [55] have analyzed the computational efficiency of the retarded-time and source-time-dominant algorithms. Both their analysis and numerical implementations found that source-time-dominant algorithm requires significantly less operations for a maneuvering rotor noise prediction $^{12}$. For a more typical rotor noise computation (up to 3 coordinate transformations and one blade passage period), both methods are comparable though the retarded-time algorithm may be slightly more efficient.

\subsubsection{Supersonic-Source Motion}

Although algorithms based upon the retarded-time formulation are robust, they have one drawback - for supersonic-source motion the integrals become singular (i.e., when $M_{r}=1$ the Doppler factor $\left|1-M_{r}\right|=0$ ) and the retardedtime equation can have multiple roots. The multiple roots for supersonicsource motion can be found without too much trouble, but the real problem is the Doppler singularity in the integrand. The necessary regularization of the integral requires both mathematical analysis and conditional code logic. As an alternative, either the collapsing-sphere formulation or the emissionsurface formulation may be used to avoid the singularity. In refs. 50 and 51

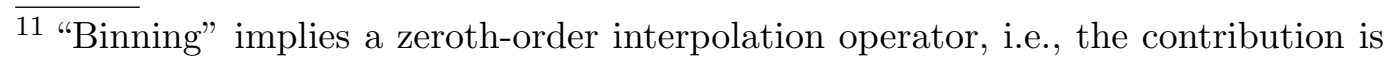
put in the nearest time "bin".

${ }^{12}$ Maneuver noise predictions are expected to require a large number of points in the time history $(>1000)$ and a large number of coordinate transformations (as many as 15) to represent the aircraft and blade motion. 
the formulation is switched for supersonic panels to utilize the efficiency and robustness of the retarded-time formulation for subsonic panels while enabling the code to handle supersonic-source motion. (Note that the Mach number $M_{r}$ used in the Doppler factor $1-M_{r}$ is the component of the source velocity in the radiation direction divided by sound speed of the undisturbed medium. This should not be confused with the local Mach number of a fluid particle.)

\subsection{Collapsing-Sphere Algorithms}

Now we turn our attention to the collapsing-sphere formulation

$$
4 \pi \phi(\mathbf{x}, t)=\int_{-\infty}^{t} \int_{\substack{f=0 \\ g=0}} \frac{Q(\mathbf{y}, \tau)}{r \sin \theta} c d \Gamma d \tau
$$

where $\theta$ is the angle between the radiation vector $\hat{\mathbf{r}}$ and the surface normal vector $\hat{\mathbf{n}}$. In equation (48), the inner integration is a line integral over the intersection of the source surface $f=0$ and the surface defined by $g=\tau-t+$ $r / c=0$. The equation $g=0$ (which is precisely the retarded-time relationship) can be interpreted as the equation of a sphere with radius $r=c(t-\tau)$ centered at the observer position $\mathbf{x}$ for a particular source time $\tau$. Recall that the observer position and time $(\mathbf{x}, t)$ are fixed during the integration in equation (48); hence, as $\tau$ approaches $t$ (in the outer integral) the radius of the sphere reduces, or collapses. For any source time $\tau$, the collapsing sphere contains all points in space that can potentially emit a signal that will be received by the observer $\mathbf{x}$ at time $t$; the intersection of the collapsing sphere $g=0$ and the data surface $f=0$ (called a $\Gamma$ curve) is the collection of source points that $d o$ emit a signal at source time $\tau$ that reaches the observer at time $t$. A schematic is shown in figure 5. The collapsing-sphere formulation does not have a Doppler singularity; however, this formulation does have a singularity in the integrand that occurs when the surface normal vector $\hat{\mathbf{n}}$ is parallel to the radiation vector $\hat{\mathbf{r}}$ (i.e., $\sin \theta=0$ ).

Farassat and Brown [56] were the first to develop a collapsing-sphere algorithm to predict the noise from thickness and loading source terms of the FW-H equation. They computed thickness and loading noise for a moving, noncompact source with realistic source geometry for both subsonic- and supersonic-source motion. In their code, Farassat and Brown evaluated the double integrals numerically with the following algorithm:

(1) Determine the initial observer time $t_{i}$ for which the collapsing sphere intersects the source surface.

(2) Choose the value of $\tau_{j}$. 
(3) Determine the intersection of the collapsing sphere $g=0$ and the source surface $f=0$. The $\Gamma$ curve is approximated by straight line segments.

(4) Compute the integrands at the ends of the line segments and evaluate the line integral over the $\Gamma$ curve by the trapezoidal rule.

(5) Advance the source time $\tau_{j}$ and repeat 3 and 4 until the collapsing sphere no longer intersects the source surface. Simpson's rule is used for the $\tau$ integration.

(6) Repeat this process for each observer time in the time history.

This algorithm is computationally demanding because a construction of the $\Gamma$ curve must be done many times for each observer time. Experience has shown that the collapsing-sphere algorithm outlined above requires 1 to 2 orders of magnitude more computer time than the mid-panel quadrature retarded-time algorithm. The retarded-time algorithm is much faster because the retarded time $\tau^{*}$ must be found only once per source panel for each observer time. Another problem experienced by Farassat and Brown was that the computed solution was oscillatory and required numerical smoothing. The $\sin \theta$ term in the denominator of equation (48) must be at least partially responsible for the numerical oscillations.

\subsection{Emission-Surface Algorithms}

Finally, we consider the general form of an emission-surface formulation:

$$
4 \pi \phi(\mathbf{x}, t)=\int_{F=0} \frac{1}{r}\left[\frac{Q(\mathbf{y}, \tau)}{\Lambda}\right]_{r e t} d \Sigma
$$

where

$$
\Lambda=\sqrt{1-2 M_{n} \cos \theta+M_{n}^{2}}
$$

and the $\Sigma$ surface $F=0$ is the emission or influence surface. The $\Sigma$ surface is the collection of points in space-time that emit signals that reach the observer at one particular observer time. The emission surface is sometimes referred to as the acoustic planform, but we reserve this terminology for when we mean the projection of the $\Sigma$ surface onto a thin surface (i.e., the helicoidal surface swept out by a propeller (e.g., see ref. 57) or the rotor disk for a helicopter rotor). The emission-surface formulation does not suffer from the Doppler singularity, but a true singularity can occur when the surface normal vector $\hat{\mathbf{n}}$ of a source point is parallel to the radiation vector $\hat{\mathbf{r}}$ and the source point is moving toward the observer at exactly sonic speed (i.e., $\Lambda=0$ ). This singularity is an indication of a caustic in the solution; Farassat and Myers [58] have shown for the $\mathrm{FW}-\mathrm{H}$ equation that the singularity from the surface source is eliminated if the quadrupole-source term is included. 
The main difficulty in the numerical evaluation of equation (49) is the construction of the $\Sigma$ surface. The $\Sigma$ surface can be constructed by using either a retarded-time computation to determine the location of the source points at the retarded time (i.e., $F=0$ is $[f=0]_{r e t}$ ) or through the process of computing the intersection of the collapsing sphere with the source surface. In either case, special care must be taken in the construction because the $\Sigma$ surface may be composed of several disjoint pieces when the source motion is supersonicjust the situation which benefits most from the use of the emission-surface formulation.

Brentner [59] has developed an alternate method for constructing the $\Sigma$ surface and performing the integration in equation (49)which is based on a computer graphics algorithm. The approach is similar to the source-time-dominant algorithm presented earlier in the retarded-time algorithms section: the source time is chosen and the corresponding observer time is computed at each grid point. If the observer times are computed and stored for each desired source time, the discrete computational data become a three-dimensional array-indices $i$ and $j$ parameterize the surface spatially and the index $k$ represents the source time. In this three-dimensional computational space, isosurfaces of observer time $t$ are, by definition, distinct realizations of the $\Sigma$ surface. Therefore, the problem of constructing the $\Sigma$ surface is equivalent to the construction of isosurfaces - an important problem in computer graphics.

Surface reconstruction must be done very efficiently and quickly to be useful for interactive data visualization. Lorensen and Cline [60] have developed the method of marching cubes, which uses a two-step approach to the surfaceconstruction problem. The first step is to locate the surface that corresponds to the level desired and approximate it with triangles. The second step is to calculate a surface normal vector at each triangle vertex. The marching-cubes method uses a divide-and-conquer approach to locate the surface in a logical cube created from eight data points (four each from two adjacent layers, as shown schematically in fig. 6).

The extension of the marching-cubes algorithm for surface integration [59] determines how the surface intersects the cube, computes the contribution to the integral from that portion of the surface and then moves (or marches) to the next cube. The topology of the surface can be determined uniquely by examining the function value (observer time in this case) at each of the cube vertices and comparing this value to the desired surface value. A table lookup is then used to determine the exact topology of the surface in the current cube. The surface is formed by a set of triangular panels that have vertices on the edges of the cubes. Brentner [59] took the value of the surface integral over each triangle as the average integrand value of the triangle vertices multiplied by the triangle area. Linear interpolation is used to determine the integrand values at the triangle vertices based on the previously computed value at the 
cube vertices. (For more detail on the marching cubes algorithm, see refs. 59 and 60.)

Because computations in the marching-cubes integration algorithm are done locally, one cube at a time, the computational problem can be easily partitioned. This separation minimizes the necessary computer storage requirements. Computation locality also enables the marching-cubes integration algorithm to perform well on parallel computer architectures. The marching-cubes algorithm has no particular problem in finding surfaces that are disjoint, hence it is well suited for use with supersonically moving sources. However, the numerical integration is accurate only to low order. Improvements in accuracy may be possible by using higher order interpolation and panel geometries other than flat triangles, but some simplicity and locality would be compromised in the process.

\section{Recent Advances}

Now that we have considered both the theory and numerical algorithms, the ground work has been laid to consider some of the recent advances in rotor noise prediction. This presentation focuses primarily on formulation development and implementation at NASA Langley Research Center from about 1990 to the present. In particular, much of the research over the past decade has focused on the prediction of impulsive noise and broadband noise. Also during the last decade the Kirchhoff formulation for moving surfaces rapidly gained popularity, but later it was demonstrated that the $\mathrm{FW}-\mathrm{H}$ equation is far superior to the Kirchhoff formula and can offer the same benefits when used with a permeable surface surrounding all the sources. These topics are each discussed in turn.

\subsection{High-Speed Impulsive Noise}

High-speed impulsive (HSI) noise is a particularly intense and annoying noise generated by helicopter rotors in high-speed forward flight. HSI noise is closely associated with the appearance of shocks and transonic flow around the advancing rotor blades and is accounted for by the $\mathrm{FW}-\mathrm{H}$ quadrupole source. Farassat and Brentner [41] have shown that after some manipulation of the formal solution the noise contribution from the quadrupole may be expressed as 


$$
\begin{aligned}
4 \pi p_{Q}^{\prime}(\mathbf{x}, t) & =\frac{1}{c} \frac{\partial^{2}}{\partial t^{2}} \int_{-\infty}^{t} \int_{f>0} \frac{T_{r r}}{r} d \Omega d \tau \\
& +\frac{\partial}{\partial t} \int_{-\infty}^{t} \int_{f>0} \frac{3 T_{r r}-T_{i i}}{r^{2}} d \Omega d \tau \\
& +c \int_{-\infty}^{t} \int_{f>0} \frac{3 T_{r r}-T_{i i}}{r^{3}} d \Omega d \tau
\end{aligned}
$$

where $p_{Q}^{\prime}(\mathbf{x}, t)$ is the acoustic pressure due to the quadrupole source. The quantity $T_{r r}$ is the double contraction of the Lighthill stress tensor $T_{i j}$ with $\hat{r}_{i}$ and $\hat{r}_{j}$; where $\hat{r}_{i}$ are the components of the unit vector in the radiation direction. In addition, $d \Omega$ is an element of the collapsing-sphere surface $g=0$; hence, equation (51) is a collapsing-sphere formulation.

The quadrupole is a volume source, hence integration over the entire collapsing sphere is indicated. In practice, however, this is not actually necessary because the Lighthill stress tensor $T_{i j}$ vanishes outside of the source region. For an in-plane observer in the far field, the collapsing sphere can be locally approximated by a right circular cylinder, as shown in figure 7 . Because the observer is assumed to be in the rotor plane (precisely where HSI noise has maximum directivity), integration in the direction normal to the rotor plane can be done independently of the observer position. Yu et al. [19] were the first to use this far-field approximation for the evaluation of quadrupole noise; however, several additional approximations were made to both the quadrupole source strength and the acoustic integrals in ref. [19]. These additional approximations are no longer necessary.

In the work of Brentner and Holland [52], the integration over the approximate collapsing-sphere surface is carried out in two parts. First, integration in the direction normal to the rotor disk is performed. They define the quadrupole source strength on the rotor plane as

$$
Q_{i j}=\int_{f>0} T_{i j} d z
$$

where $z$ is understood to be in the direction normal to the rotor disk and the $z$ integration is only performed outside of the rotor blade; $Q_{i j}$ is nonzero only in the region near the rotor blade planform and extends ahead of the leading edge, behind the trailing edge and off the blade tip. By using relation (52), equation (51) may be rewritten as 


$$
\begin{aligned}
4 \pi p_{Q}^{\prime}(\mathbf{x}, t) & =\frac{1}{c} \frac{\partial^{2}}{\partial t^{2}} \int_{-\infty}^{t} \int_{\substack{f^{+}=0 \\
g=0}} \frac{Q_{r r}}{r} d \Gamma d \tau \\
& +\frac{\partial}{\partial t} \int_{-\infty}^{t} \int_{\substack{f^{+}=0 \\
g=0}} \frac{3 Q_{r r}-Q_{i i}}{r^{2}} d \Gamma d \tau \\
& +c \int_{-\infty}^{t} \int_{\substack{f^{+}=0 \\
g=0}} \frac{3 Q_{r r}-Q_{i i}}{r^{3}} d \Gamma d \tau
\end{aligned}
$$

where $f^{+}=0$ represents the rotor-disk plane. The intersection of the collapsing sphere with the rotor plane results in a $\Gamma$ curve.

\subsubsection{Subsonic formulation}

In the development of a subsonic quadrupole formulation, equation (53) is transformed from a collapsing-sphere formulation to a retarded-time formulation (using the relations described earlier). When the time derivatives are taken inside the retarded-time integrals [45], the quadrupole formulation may be written

$$
4 \pi p_{Q}^{\prime}(\mathbf{x}, t)=\int_{f^{+}=0}\left[\frac{K_{r 1}}{c^{2} r}+\frac{K_{r 2}}{c r^{2}}+\frac{K_{r 3}}{r^{3}}\right]_{r e t} d S
$$

where

$$
\begin{aligned}
K_{r 1} & =\frac{\ddot{Q}_{r r}}{\left(1-M_{r}\right)^{3}}+\frac{\ddot{M}_{r} Q_{r r}+3 \dot{M}_{r} \dot{Q}_{r r}}{\left(1-M_{r}\right)^{4}}+\frac{3 \dot{M}_{r}^{2} Q_{r r}}{\left(1-M_{r}\right)^{5}} \\
K_{r 2} & =\frac{-\dot{Q}_{i i}}{\left(1-M_{r}\right)^{2}}-\frac{4 \dot{Q}_{M r}+2 Q_{\dot{M} r}+\dot{M}_{r} Q_{i i}}{\left(1-M_{r}\right)^{3}} \\
& +\frac{3\left[\left(1-M^{2}\right) \dot{Q}_{r r}-2 \dot{M}_{r} Q_{M r}-M_{i} \dot{M}_{i} Q_{r r}\right]}{\left(1-M_{r}\right)^{4}} \\
& +\frac{6 \dot{M}_{r}\left(1-M^{2}\right) Q_{r r}}{\left(1-M_{r}\right)^{5}}
\end{aligned}
$$

and

$$
K_{r 3}=\frac{2 Q_{M M}-\left(1-M^{2}\right) Q_{i i}}{\left(1-M_{r}\right)^{3}}-\frac{6\left(1-M^{2}\right) Q_{M r}}{\left(1-M_{r}\right)^{4}}+\frac{3\left(1-M^{2}\right)^{2} Q_{r r}}{\left(1-M_{r}\right)^{5}}
$$

Equation (54), together with the definitions of $K_{r 1}, K_{r 2}$ and $K_{r 3}$, are referred to as formulation Q1A. Formulation Q1A does not require numerical time 
differentiation of the integrals and, as a retarded-time formulation, is well suited for subsonic source motion. Aside from the problem geometry, only the time-dependent value of $Q_{i j}$ is required as input. Brentner has implemented formulation Q1A in a new version of the WOPWOP noise prediction code [44] now called WOPWOP +. (Full details of the derivation are given in ref. 45.)

\subsubsection{Supersonic Formulation}

To develop a supersonic quadrupole formulation, equation (53) can be transformed from a collapsing-sphere formulation to an emission-surface formulation. The emission-surface formulation is appropriate for supersonic-source motion because it does not have a Doppler singularity $\left|1-M_{r}\right|$ in the denominator of the integrand. Farassat and Brentner [61] took the time derivatives inside the integrals by recognizing that the entire rotor plane can be considered as the emission-surface, rendering the limits of integration independent of time. Next they changed coordinates from a frame fixed to the undisturbed medium to a frame always aligned with the rotor blade, (i.e., $(\mathbf{x}, t) \rightarrow(\boldsymbol{\eta}, \tau)$ ). The effect of this operation on the tensor $\mathbf{Q}$ with components $Q_{i j}$ is

$$
\begin{aligned}
\left.\frac{\partial\left[Q_{i j}\right]_{r e t}}{\partial t}\right|_{\mathbf{x}} & =\left[\left.\frac{\partial}{\partial \tau}\right|_{\mathbf{x}} Q_{i j}\right]_{r e t} \\
& =\left[\left.\frac{\partial Q_{i j}}{\partial \tau}\right|_{\boldsymbol{\eta}}-\mathbf{V} \cdot \nabla_{\boldsymbol{\eta}} Q_{i j}\right]_{r e t} \\
& \equiv\left[L_{\tau} Q_{i j}\right]_{r e t}
\end{aligned}
$$

where $\boldsymbol{\eta}$ is the position vector in the rotating frame and $\tau$ is the source time. Here $\mathbf{V}=\partial \boldsymbol{\eta} / \partial \tau$ is the velocity of the point $\boldsymbol{\eta}$ specified in the frame fixed to the undisturbed medium. We note that $\mathbf{V}$ has no component normal to the rotor plane. It is important to recognize that when we refer to $\left.Q_{i j}\right|_{\eta}$ we really mean that the components of the tensor $Q_{i j}$ are represented in a coordinate system is instantaneously aligned with the rotating frame. Thus equation (55) provides the time derivative of $\left.Q_{i j}\right|_{\mathbf{x}}$ in the stationary frame in terms of $\left.Q_{i j}\right|_{\eta}$ which is specified in the coordinates of the moving frame. Using the operator notation $L_{\tau}$ defined in equation (55), the final emission-surface formulation may be written

$$
\begin{aligned}
4 \pi p_{Q}^{\prime}(\mathbf{x}, t) & =\frac{1}{c^{2}} \int \frac{1}{r} \hat{r}_{i} \hat{r}_{j}\left[L_{\tau}^{2} Q_{i j}\right]_{r e t} d \Sigma \\
& +\frac{1}{c} \int \frac{1}{r^{2}}\left[3 \hat{r}_{i} \hat{r}_{j} L_{\tau} Q_{i j}-L_{\tau} Q_{i i}\right]_{r e t} d \Sigma \\
& +\int \frac{1}{r^{3}}\left[3 Q_{r r}-Q_{i i}\right]_{r e t} d \Sigma
\end{aligned}
$$


Notice that the operator $L_{\tau}$ only operates on $Q_{i j}$ because $\hat{r}_{i}$ and $\hat{r}_{j}$ do not depend upon $t$ or $\tau$. We refer to equation (56) as formulation Q2. Examining the definition (55) and equation (56), we realize that formulation Q2 is a singularity free expression for supersonic quadrupole noise. Note that equation (56) has second space and time derivatives of $Q_{i j}$ as well as first space derivatives in the rotor plane. These quantities are available in the CFD postprocessor that is used to compute $Q_{i j}$ for acoustic calculations. As it stands, formulation Q2 is valid for subsonic and supersonic quadrupole noise prediction for helicopter rotors in hover or forward flight. (Full details are given in ref. 61.)

Formulation Q2 has been implemented in a demonstration code ${ }^{13}$ known as WOPWOP2 +. WOPWOP2 + differs significantly from WOPWOP $+[44,45$, $52]$ in that it uses an emission-surface formulation to compute thickness and loading noise, as well as the quadrupole noise. The construction of the emission surface and subsequent integration over the emission surface is performed using the method of marching cubes integration developed by Brentner [59]. In ref. 61, it is shown that the subsonic (WOPWOP+) and the supersonic (WOPWOP2+) quadrupole formulations give identical results when the quadrupole grid extent is the same.

Ianniello $[62,63]$ has also developed quadrupole noise prediction codes that integrate the FW-H equation on a supersonic rotating domain. The main new feature in Ianniello's work is that he has developed a sophisticated emissionsurface construction and integration scheme [64]. In this scheme Ianniello characterizes each radial section on the grounds of the distribution of sources with multiple emission time(s) of the leading and trailing edges, into four separate regions and then treats each region appropriately (including a self-adaptive refinement scheme). Ianniello uses his algorithm to compute the far-field approach of Brentner [45] and has also performed the full volume quadrupole integration. The integration results from Ianniello's latest computations [63] are very good (as shown later in figure 9) and the computational time seems quite reasonable.

\subsubsection{HSI Noise Predictions}

We now present HSI noise calculations for a two-blade model-scale UH-1H rotor tested in hover with tip Mach numbers 0.88, 0.9, 0.925 and 0.95 (See ref. 65 for test information). The quadrupole grid in these computations extends $1.86 R$ beyond the blade tip for all the WOPWOP2 + calculations shown in figure 8 . For comparison, we have also shown the signature predicted by WOPWOP + which includes quadrupole sources only up to the sonic circle ${ }^{14}$.

\footnotetext{
$\overline{13}$ WOPWOP2 + had only hover capability.

${ }^{14}$ The sonic circle is the radius at which the quadrupole source grid has a velocity $\Omega R$ equal to the quiescent sound speed $c$.
} 
The agreement between the WOPWOP2+ signature and the measured data is excellent and better than that of WOPWOP + for each case. For the more intense cases $\left(M_{H}>0.90\right)$, the agreement of the WOPWOP2+ prediction with the measured acoustic pressure signature is not fully satisfactory because the WOPWOP2+ prediction overpredicts the negative peak pressure. This is apparent in figure 8 for the times between the WOPWOP + and the WOPWOP2 + shock locations. This overprediction is related to the numerical second derivative computation of quadrupole source tensor $Q_{i j}$. More careful treatment of the numerical integration required to form $Q_{i j}$ and potentially integrating on the actual collapsing sphere together with improved numerical differentiation of $Q_{i j}$ should eliminate this error. Ianniello's algorithm constructing the emission surface gives a better result for the $M_{H}=0.95$ case (see figure 9).

To demonstrate the forward-flight capability of the WOPWOP + code, Brentner [45] made a comparison between predicted and measured results for a four-blade swept-tip rotor tested in the German-Dutch wind tunnel (DNW). For this comparison, a microphone located in the rotor plane at an azimuth of $\psi=150^{\circ}$ was used. The experiment is described in the report by Visintainer et al. [66]

The full potential solver FPRBVI [67] was used to compute the unsteady flow field around the rotor. The CFD solution was stored at every degree of rotor azimuth for the quadrupole source strength computation. The results of the forward-flight noise prediction are shown in figure 10. The predicted acoustic pressure is compared with the experimental data; the quadrupole contribution is also shown to indicate its relative magnitude. Although the CFD calculation used a rather coarse grid, the agreement is good.

\subsection{Blade-Vortex-Interaction (BVI) Noise}

BVI noise is generated when a rotor blade intersects a previously shed tip vortex, as shown in figure 11. BVI noise can occur continually in some flight regimes, e.g., in descending flight. In this situation, the noise is very loud and annoying to the nearby community. When BVI occurs, it is the most annoying (hence most significant) source of rotor noise. Essentially, BVI noise can be classified as an impulsive loading noise because the loading (dipole) source term in the FW-H equation accounts for the noise. Much research has been done on this noise mechanism since the early seventies. Schmitz [68] and Lowson $[69,70]$ both have good discussions on BVI noise in their respective articles on helicopter rotor noise. More recently, Yu also presented a review of rotor blade-vortex interaction noise [71]. In light of these articles, this section will be limited to a presentation of the current state of BVI noise prediction 
and a brief discussion of a few of the key contributions to BVI noise modeling and prediction.

Most of the effort in the last several years to predict BVI noise has gone into modeling the transient blade surface pressure fluctuations in the interaction process. This effort is justified by the fact that when measured blade surface pressure fluctuations are used in acoustic codes (e.g., WOPWOP), the measured and predicted noise agree very well (See figure 12). At present, the task of calculating the blade surface pressure is quite a difficult problem but within the reach of current technology. The primary difficulty in the computation of the blade surface pressure is the determination of the precise position and strength of the vortical wake shed by the rotating blades. Another challenge in the calculation of helicopter rotor airloads is the complex aerodynamic and structural dynamics coupling. All of these elements must be solved accurately in order to obtain an accurate acoustic prediction.

To illustrate the current state-of-the-art, we now give some comparisons of predicted and measured acoustic pressure signatures for BVI noise. These calculations were performed at the NASA Langley Research Center [72,73] with codes from the rotorcraft noise prediction system known as TRAC (TiltRotor Aeroacoustic Codes). Figure 13 shows a flowchart of the computer codes that form the elements of the TRAC.

TRAC is a system of prediction codes that can be used to predict the noise for both helicopter and tiltrotor vehicles. In the results presented below, the combination of CAMRAD.Mod1, HIRES and WOPWOP are used for BVI noise prediction. The CAMRAD code was originally written by Wayne Johnson [74] primarily for rotorcraft performance calculation. CAMRAD.Mod1 is a highly modified version of CAMRAD with enhanced capability [72]. This code gives a rotor wake description at the fairly low azimuthal resolution of 10 degrees. The HIRES code postprocesses the low resolution output of CAMRAD.Mod1 to generate a high resolution description of the rotor wake, blade motion and sectional loads, using classical aerodynamics. The high resolution wake description is typically determined at 0.5 to $1 \mathrm{deg}$. resolution in rotor azimuth angle and between 75 and 100 radial stations. Much ingenuity and experience has gone into the development of these two codes.

The first prediction to be shown from TRAC is for a 40 percent dynamically and Mach scaled model of a BO-105 main rotor. The four-bladed rotor is $4 \mathrm{~m}$ in diameter, hingeless and has a preconing of $2.5 \mathrm{deg}$. at the hub. The blades have a rectangular planform with a $0.121 \mathrm{~m}$ chord and $-8 \mathrm{deg}$. linear twist. The aerodynamic and acoustic measurements were performed in the open test section of the German-Dutch wind tunnel (DNW) [75]. Figure 14 shows the predicted and measured BVI sound pressure signature at one microphone location below the advancing side of this model rotor. In CAMRAD.Mod1, the

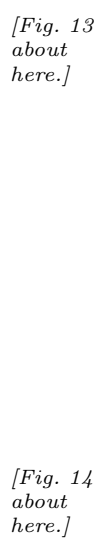


tip vortex was modeled as a combination of main- and secondary-tip vortices. This model, which was based upon experimental observations, improved the prediction of the blade-vortex interaction process leading to the relatively good agreement between both measurement and predicted acoustic pressure signatures in figure 14. In particular, the location and amplitude of the sharp BVI pulses are predicted well.

Figure 15 shows another example of BVI noise prediction for a tiltrotor model operating in helicopter mode [72,73]. The $1.74 \mathrm{~m}$ diameter rotor has 3 blades, solidity $\sigma=0.114$ and is a 15 percent model of the JVX tiltrotor. The blade tip chord is $0.091 \mathrm{~m}$ and the blade twist is $-47.5 \mathrm{deg}$. This model was tested in the Langley $14 \times 22$ foot subsonic wind tunnel [76]. We have shown two acoustic predictions with two different tip vortex models. The model with no roll-up has a single strong tip vortex. The corresponding BVI noise signature, figure 15(c), considerably overpredicts the measured signature shown in figure 15(a). The tip-vortex model with roll-up has two vortices at and near the tip. The corresponding BVI noise signature, figure 15(b), agrees better with the measured signature.

The above results - which are representative in both the level of detail and accuracy of predictions achieved by several research groups - clearly indicate that BVI noise prediction is sensitive to accurate prediction of the rotor loading. The prediction of the transient blade surface pressure due to BVI must agree with experimental tip-vortex structure and roll-up behavior. At present, satisfactory prediction of BVI noise is possible using classical aerodynamics to obtain the blade surface pressure. Future developments in unsteady aerodynamics based on the numerical solution of the Navier-Stokes equations should provide further improvements in BVI noise prediction.

Singh and Baeder $[77,78]$ used the WOPWOP+ code together with a NavierStokes code to study the importance of transonic effects on the acoustics of BVI noise. For BVI prediction, the in-plane approximation is too restrictive because BVI radiation normally occurs below the rotor plane and the collapsing sphere is at some angle to the rotor plane. Singh modified the quadrupole preprocessor by dividing the aerodynamic flow field above and below the rotor plane into several layers to minimize the error associated with integration in the direction normal to the rotor plane. Singh and Baeder found that transonic effects cause the BVI noise radiation to be stronger in the rotor plane, but very little difference was noted in the region of maximum BVI noise intensity (approximately 45 degrees below the rotor plane).

Some joint efforts of the past few years dealing with BVI noise are of particular interest. Not completely understanding the discrepancies noted in comparison between various BVI noise predictions, Caradonna et al. [79] formed a BVI working group to try to understand the problem through a simpler type of 
BVI. These researchers tested their BVI noise prediction tools on the problem of an independently generated vortex (generated by a wing upstream of the rotor) interacting with a rotor in forward flight. ${ }^{15}$ An experiment was run by Kitaplioglu and Caradonna [80] that simultaneously measured both pressure on the rotor blades and the acoustic pressure at several microphone locations. This was followed by a blind prediction of both the BVI airloads and noise by all the groups involved. Most of the predictions gave generally good results even though the computational tools used were somewhat different. The outcome of this effort supports the conclusion that the acoustic prediction methodology is fairly mature while much remains to be done to improve the determination of the wake and tip-vortex geometry and strength.

In 1994, a joint French/German/US rotor noise test was performed in the German-Dutch wind tunnel (DNW) [81-83]. This higher-harmonic control aeroacoustic rotor test (HART) included measurement of the both rotor aerodynamics and acoustics, along with some wake measurements, for BVI noise conditions for a rotor operating with and without higher-harmonic control. The HART dataset is an extremely valuable asset for the refinement of BVI noise modeling because high quality aerodynamic, acoustic and wake measurements are all available in a single test. In particular, it was determined that the primary mechanism by which HHC (Higher Harmonic Control) increases or decreases the BVI noise is the modification to the rotor wake and blade tip vortices. Recently, a follow-on test (HART II [84]) was completed. In the HART II test the rotor wake was measured in much more detail, therefore, this dataset promises to shed light on some of the rotor wake/tip vortex modeling problems that continue to persist. Although HHC was studied in the HART tests, several other noise reduction concepts, both active and passive, have been studied over the past decade. Ref. 71 provides a discussion of BVI noise reduction concepts and their analysis.

\subsection{Broadband Noise Prediction}

Helicopter rotors generate broadband noise that occurs in the frequency range where the human ear is most sensitive ( 1 to $5 \mathrm{kHz}$ ). For this reason much effort has gone into understanding the mechanisms of broadband noise generation and its prediction. Currently, the mechanisms of broadband noise generation are well understood. Great strides have been made in the prediction of broadband noise of helicopter rotors. Howe, Amiet and coworkers, and Brooks and coworkers have made significant contributions to the understanding and prediction of broadband noise. Howe and Brooks are currently active in the field;

\footnotetext{
${ }^{15}$ An externally generated vortex simplifies the problem somewhat, since the vortex trajectory and strength are known a priori.
} 
Howe's work has emphasized trailing edge (TE) noise prediction while Brooks and coworkers have concentrated on all aspects of rotor broadband noise prediction.

For a brief history of rotor broadband noise prediction, see Brentner and Farassat [1]. Here we concentrate on more recent progress. A recent publication by Brooks and Burley [85] gives a comprehensive account of rotor broadband noise prediction with comparison to model data. Essentially all broadband noise is generated by random loading on the rotor blade. Thus, the source term is the loading term in the $\mathrm{FW}-\mathrm{H}$ equation. In the helicopter rotor noise spectrum, the mid- to high-frequency range is generally of broadband nature. The mid-frequency broadband noise is produced by blade-wake interaction (BWI). BWI noise is produced when the rotor blade encounters the wake turbulence, usually of preceding blades. This situation appears in level flight and mild climb conditions. The high-frequency broadband noise is mostly selfnoise, i.e., the noise produced by boundary layer turbulence and the shedding of turbulent eddies at the trailing edge.

At present, the BWI noise prediction proposed by Brooks and Burley [86] is semi-empirical and based on the measured blade response to wake turbulence. To formulate their prediction method, an extensive aeroacoustic database was used. Blade response coherence functions were found and used in a noise radiation formula developed by Amiet [87]. This approach was selected because of the inhomogeneous nature of turbulence in the rotor wake and the dependence of its statistics on operating conditions. Burley and Brooks claim that they obtain very good agreement in directivity and spectra with measured noise for a large range of flight conditions. This approach could be improved further because Amiet's acoustic result is only valid for an airfoil in uniform rectilinear motion. In fact in Amiet's work it is possible to get a closed form solution for the noise spectrum in terms of the turbulent statistics-because of this assumption. For a rotating blade Amiet's result is valid only approximately even when strip theory is used. Also in Amiet's work, aerodynamics and acoustics are inherently coupled.

For situations where detailed surface pressure information are available, either from computation or experiment, another approach is required for which the aerodynamics and acoustics are uncoupled. An obvious choice is the acoustic analogy. Because one looks for a relation between the noise spectrum and the statistics of surface pressure fluctuations, the choice of the form of the solution of the FW-H equation is important. Casper and Farassat [88] have derived a new solution of the $\mathrm{FW}-\mathrm{H}$ equation called formulation $1 \mathrm{~B}$ which is particularly suitable for broadband noise prediction. Casper and Farassat have shown that broadband noise can be predicted in the time domain using this formulation. They have calculated the broadband noise spectrum of an airfoil interacting with homogeneous turbulence in an experiment reported by Amiet 
[87]. Complete agreement with results from Amiet's frequency domain method was obtained over a wide range of tunnel speeds. It should be mentioned that there was also generally good agreement between measured and predicted noise spectra. Formulation 1B also appears to be highly suitable for trailing edge noise prediction.

The self noise of helicopter rotor blades is generated by several mechanisms as discussed by Brooks and Burley [85]. These include trailing edge (TE) noise from turbulent boundary layer or separated flow interaction with the edge, laminar boundary layer vortex shedding and blade tip noise. (See figure 16.) Trailing edge noise generation has been studied for over forty years and the literature on this subject is extensive. Two important recent publications on this subject at low Mach numbers are by Howe $[89,90]$. The physics of the TE noise generation can be described as follows: as the turbulent eddies pass over the trailing edge, the surface pressure fluctuations produced by the eddies radiate noise at a much higher efficiency than that of the quadrupole sources of the turbulent eddies alone. Therefore, the surface dipole noise can be orders of magnitude larger than the quadrupole sources that caused the surface pressure fluctuations. ${ }^{16}$ From the point of view of the acoustic analogy, one requires this pressure fluctuation as a function of space and time variables to perform acoustic predictions. However, both obtaining experimental data or computing these pressure fluctuations using CFD are very difficult. Obtaining experimental data is difficult because small pressure transducers with high-frequency response are needed near the TE where the airfoil thickness is generally very small. Turbulence simulations in recent years have advanced considerably and predictions of unsteady pressure fluctuations are gradually becoming available. However, there is the question of what acoustic formulation to use when the surface pressure data are available. We will address this question below.

In the absence of measured or calculated surface pressure fluctuation data near the TE of the blade, much effort has gone into the understanding of the physics of its generation from first principles $[89,90]$. It seems natural to use an incompressible turbulent flow simulation for TE noise. Howe [89] has shown that the use of the Green's function in the unbounded domain for high frequency TE noise prediction can lead to incorrect results. The cause of the error is that in incompressible flows, any signal travels at infinite speeds while acoustic waves travel at finite speed. Thus, the pressure disturbance from the TE is felt immediately on the upstream section of the blades. Also, the primary characteristic of the acoustic wave propagation is missed in incompressible fluid computation. Howe proposes to use a Green's function whose normal

\footnotetext{
$\overline{{ }^{16} \text { If the }}$ unsteady surface fluctuations are computed from an incompressible CFD solution, then a Green's function appropriate to the problem geometry (as opposed to the Green's function for unbounded space) should be used [89-91].
} 
derivative is zero over the airfoil and shows that under some conditions, usually satisfied in TE noise generation, this is the appropriate Green's function for acoustic calculations. It is obvious that this question of the selection of the Green's function for the TE noise prediction does not appear if one uses compressible turbulence simulation.

Brooks and Burley [85] have proposed and implemented models of several mechanisms of rotor high-frequency broadband noise prediction in the NASA Langley's TiltRotor Aeroacoustic Codes (TRAC) system [92, 93]. The noise spectra of all these models are based on the results of Ffowcs Williams and Hall [94] in Mach number dependence and spectral attenuation. They also depend on a function of several variables such as Strouhal number, various Reynolds numbers and the Mach number. These authors are attempting to compute the high-frequency broadband rotor noise in its full complexity for a rotor operating in the realistic environment of forward flight. The model of TE noise generation of Ffowcs Williams and Hall is too idealistic for the situation that Brooks and Burley consider. Nevertheless, it appears that by using several adjustable degrees of freedom in their models, based on measured aerodynamic and acoustic data, Brooks and Burley have developed a very useful tool for the helicopter industry to predict the self noise of helicopter rotors.

A number of researchers have used turbulence simulation for broadband noise prediction (e.g.,91,95-97). In general, large eddy simulation (LES) of the turbulent eddies is now within reach of acousticians for noise prediction if relatively simple geometries are assumed. However, LES is usually computed for an incompressible fluid. It is believed that LES resolves the noise generating eddies over a wide range of length scales in engineering applications. Reynolds-Averaged Navier-Stokes (RANS) equation-based CFD can not adequately supply the surface pressure fluctuations needed for broadband noise prediction. Direct numerical simulation (DNS) of turbulence could supply this information, but is too computationally demanding and uses much more computer resources than LES. The trend in the computation of the unsteady surface pressure is toward more dependence on LES or DNS of the flow over the airfoil or blade surface. The inclusion of compressibility in LES and DNS is highly desirable because it provides a more realistic modeling of wave propagation in the vicinity of the surface. The prediction of the broadband component of helicopter rotor noise in forward flight with realistic blade geometry and kinematics using LES or DNS is not feasible today. We are perhaps a decade away from reaching this goal.

What are our options to compute the radiated noise once the blade surface pressure fluctuations are available? (We are assuming that the observer is not at such a distance that propagation effects such as absorption are important.) The current choices are CFD based computational aeroacoustics (CAA) or the acoustic analogy based on the FW-H equation. The CAA approach 
can be based on finite difference [98] or finite element [99] methods. Both of these methods require discretization of the space from the source to the observer. The spatial grid size is governed by the smallest acoustic wavelength. In addition, special absorbing far-field boundary conditions must be used and numerical stability conditions must be met. Altogether finite difference and finite element methods use much more computer resources than acoustic analogy based methods. The FW-H equation with a penetrable data surface has shown good accuracy and efficiency in the prediction of rotor noise. The recent work of Casper and Farassat [88] has shown that the FW-H equation can be also used for broadband noise prediction in the time domain (See also Singer et al. [91]) Two of the most important advantages of the acoustic analogy approach are: i) the analytic nature of the method allows derivation of a closed form solution of the $\mathrm{FW}-\mathrm{H}$ equation satisfying the far-field boundary condition. The form of the solution can be tailored to the specific problem for efficiency and accuracy, e.g., formulations 1A and 1B; ii) in general, very complex geometry and kinematics of the rotor blades can be modeled exactly rather easily when the acoustic analogy is used. The spatial grid generation of this problem for finite difference and finite element methods is a very difficult problem today.

Although both CAA and acoustic analogy approaches will be used in the future, the authors believe that the acoustic analogy will meet most needs of design engineers particularly if source kinematics are complex. Semi-empirical methods such as those proposed by Brooks and Burley will remain important tools for design engineers at least until compressible turbulence simulation has reached a mature and reliable stage.

\subsection{Ffowcs Williams - Hawkings Equation as a Kirchhoff Formulation}

Recently the FW-H equation has been used in a new way for the computation of rotor noise through the application of the $\mathrm{FW}-\mathrm{H}$ equation on a permeable or porous surface. This is an example of the development of an alternative but equivalent source description. In rotor noise prediction, the surface $f=0$ has usually been assumed to be coincident with the rotor blade surface and impenetrable $\left(u_{n}=v_{n}\right)$. A relaxation of that assumption is useful because it enables consideration of either conveniently placed fictitious surfaces or physical surfaces that permit flow through them.

For a permeable surface (fictitious or physical), equation (18) is the appropriate expression of the $\mathrm{FW}-\mathrm{H}$ equation. It is clear from both the original Ffowcs Williams and Hawkings paper [11] and later work [100, Chapter 11, Sec 10] that Ffowcs Williams understood the value of using equation (18) on a permeable surface. Recently di Francescantonio [101] implemented this 
form of the $\mathrm{FW}-\mathrm{H}$ equation for rotor noise prediction. At nearly the same time, Brentner and Farassat [102] demonstrated the relationship between the FW-H equation and the Kirchhoff equation for moving surfaces. Many others have followed suit by quickly adopting the use of the $\mathrm{FW}-\mathrm{H}$ equation on a permeable surface [103-105].

One question to be considered is "What is the advantage of a fictitious surface that is not coincident with the physical body?" Although it may not be immediately obvious from equations (18) or (19), any physical acoustic sources enclosed by the $f=0$ surface only contribute through the surface-source terms. Any physical sources of sound or propagation effects outside $f=0$ contribute through the volume source term. Hence, if we can enclose all physical sources inside $f=0$ we have no contribution from the volume source-i.e., the quadrupole can be legitimately neglected. Without the quadrupole, a significant computational savings is realized because the volume integration is no longer required. Furthermore, the amount of flow-field data required for a surface integration is much less than that for an integration of the volume surrounded by the surface.

The key, which enables use of the $\mathrm{FW}-\mathrm{H}$ equation on a permeable surface, is the availability of an accurate flow-field description from CFD. Only recently has CFD matured to a point where it can provide sufficiently accurate, unsteady flow-field data on the integration surface. Even so, CFD computations for a rotor in forward flight are extremely demanding. Thus the coupling of the FW-H equation and CFD provides a mutually beneficial approach to the computation of noise. The CFD computation only is needed in the acoustic source region - not all the way to the observer - and the FW-H equation provides an efficient method to predict the sound field away from the source region. Through the use of the permeable surface formulation of the FW-H equation, the acoustic calculations can be made computationally efficient even for complicated, nonlinear acoustic sources.

To demonstrate the robustness and accuracy of the permeable surface application of the $\mathrm{FW}-\mathrm{H}$ equation, a comparison between the predicted acoustic pressure and experiment for a hovering rotor is shown in figure 17. In the figure, the result for a WOPWOP+ (subsonic quadrupole) prediction is also shown. The same CFD calculation was utilized for the input data for both predictions. Two permeable surface $\mathrm{FW}-\mathrm{H}$ computations are show in figure 17. First, an integration surface coincident with the rotor blade is used to predict thickness and loading noise. Second, an integration surface located approximately 1.5 chord lengths away from the blade is used to predict the total noise. Note that the thickness noise predictions from WOPWOP + and $\mathrm{FW}-\mathrm{H}$ are identical and there is only a small difference in the predicted loading noise. The total noise, which includes the effects of the quadrupole, is also in very close agreement even though the volume used in WOPWOP + is not 
identical to the region enclosed in the $\mathrm{FW}-\mathrm{H}$ permeable-surface integration.

\subsubsection{Problems with the Kirchhoff Formulation}

The Kirchhoff formulation for moving surfaces is an alternative formulation that has been used for rotor noise prediction over the past decade. The Kirchhoff formulation gained rapid acceptance shortly after its publication by Farassat and Myers in 1988 [106] precisely because it appeared to offer the same benefits just presented for the permeable-surface application of the $\mathrm{FW}-\mathrm{H}$ equation. Nevertheless, recent work by Brentner and Farassat [102] and Singer et al. [91] have shown that the Kirchhoff formulation is unreliable for aeroacoustic problems in practice. This observation is sufficiently important that some explanation is desirable.

An embedding procedure similar to that used to derive the $\mathrm{FW}-\mathrm{H}$ equation above was applied to the wave equation by Farassat and Myers to derive the Kirchhoff formulation for moving surfaces. The generalized wave equation, which is the governing equation for the Kirchhoff formulation, becomes

$$
\begin{aligned}
\square^{2} p^{\prime}(\mathbf{x}, t)= & -\left(\frac{\partial p^{\prime}}{\partial t} \frac{M_{n}}{c}+\frac{\partial p^{\prime}}{\partial n}\right) \delta(f) \\
& -\frac{\partial}{\partial t}\left(p^{\prime} \frac{M_{n}}{c} \delta(f)\right)-\frac{\partial}{\partial x_{i}}\left(p^{\prime} \hat{n}_{i} \delta(f)\right) \\
\equiv & Q_{k i r}
\end{aligned}
$$

where $M_{n}=v_{n} / c$. In this equation $p^{\prime}$ must be compatible with the wave equation: hence, equation (57) is valid only in the region of the fluid in which the wave equation is the appropriate governing equation.

Through the utilization of the continuity and momentum equations we can rewrite the permeable surface form of the $\mathrm{FW}-\mathrm{H}$ equation (18), as

$$
\begin{aligned}
& \square^{2} p^{\prime}(\mathbf{x}, t)=Q_{k i r}+\frac{\bar{\partial}^{2}}{\partial x_{i} \partial x_{j}}\left[T_{i j} H(f)\right] \\
& +\frac{\partial}{\partial t}\left[p^{\prime}-c^{2} \rho^{\prime}\right] \frac{M_{n}}{c} \delta(f)+\frac{\partial}{\partial t}\left[\left(p^{\prime}-c^{2} \rho^{\prime}\right) \frac{M_{n}}{c} \delta(f)\right] \\
& -\frac{\partial}{\partial x_{j}}\left[\rho u_{i} u_{j}\right] \hat{n}_{i} \delta(f)-\frac{\partial}{\partial x_{i}}\left[\rho u_{i} u_{n} \delta(f)\right] .
\end{aligned}
$$

This form of the FW-H equation highlights the differences between the Kirchhoff formulation and the FW-H equation. Notice that all the additional source terms in equation (58) are second-order terms in perturbation quantities outside of the source region (i.e., $p^{\prime}-c^{2} \rho^{\prime} \ll 1$ and $\rho u_{i} u_{j} \ll 1$ ). For linear wave 
propagation, each of these terms would be identically zero and the Kirchhoff formulation and the FW-H equation would be in complete agreement. In other words, if the input data identically satisfies the wave equation, then the $\mathrm{FW}-\mathrm{H}$ equation and Kirchhoff equation for a moving surface are identical - but this is seldom the situation in practice where CFD results are used as input data. ${ }^{17}$ A more detailed comparison and discussion are found in ref. 102.

To illustrate how the Kirchhoff formulation can give misleading results, consider a series of cylindrical integration surfaces that enclose a hovering rotor blade, as shown in figure 18. In figure 19, the acoustic pressure for an in-plane observer has been computed using both the FW-H equation and the Kirchhoff formulation for each of the integration surfaces. Both methods agree reasonably well with the data when the integration surfaces are more than about 0.7 chords from the blade surface. The predicted acoustic pressure from the Kirchhoff computations for integration surfaces that are closer to the blade are unrealistic. In contrast, the acoustic pressure predicted by the $\mathrm{FW}-\mathrm{H}$ equation is well behaved and is modified only by the fact that not all of the "quadrupole" noise source is included when the integration surface is too close to the blade and the volume source term in equation (18) has been neglected.

A second example illustrates another problem that can occur with the Kirchhoff formulation. In this case, consider the viscous flow past a circular cylinder, as shown in figure 20. In this situation it is expected that the vortices shed by cylinder have a very small contribution to the sound produced, hence the acoustic signal should be relatively unaffected by the placement of the integration surface. Figure 21 shows that this is indeed the case for the FW-H computation, but the Kirchhoff computation is entirely erroneous. This example is significant for rotor noise prediction because rotor wakes inevitably must pass through the integration surface.

These two examples demonstrate numerically that the Kirchhoff formulation is not reliable for rotor noise prediction. Fortunately, from a computational point of view, there is very little difference between the methods. All of the computational advantages originally sought from the Kirchhoff method are available using the FW-H equation. Furthermore, all of the physical insight that the FW-H equation traditionally has provided is also still available.

\subsection{Vehicle Noise Prediction}

As the models for aerodynamically generated sound have been developed and become more accurate and sophisticated, some effort has shifted to consider-

$\overline{17}$ Generally the input data is the solution to the Navier-Stokes or Euler equations rather than the wave equation. 
ation of the rotorcraft as a vehicle rather than just as a collection of independent, isolated rotors. Until recently, the individual noise sources from the rotor have been treated separately - with only minimal recognition that real rotorcraft have a fuselage that can interact with both the flow and acoustic radiation. Furthermore, real rotorcraft maneuver along sometimes complicated flight paths to perform their mission. These aspects of rotorcraft flight can have a significant impact upon the radiated noise. Ultimately a complete system noise prediction needs to include the effects of acoustic scattering and maneuver. These topics are discussed in the following subsections.

\subsubsection{Acoustic Scattering}

Rarely in the development of aeroacoustic noise models is the rotorcraft fuselage taken into account. The fuselage acts as a scattering body in the path of noise propagation. In general, the fuselage can cause reflections, scattering, diffraction and shielding of the sound - depending on the observer location. The rotor blade passage frequency $(\mathrm{BPF})$ is generally low for rotorcraft, hence the wavelength of the low-frequency noise is quite large compared to the physical dimensions of the fuselage cross section and the influence of the body is minimal. Impulsive noise contains a much higher frequency content, thus the fuselage is a much more efficient scatterer of BVI and HSI noise. Furthermore, the location of BVI and HSI noise generation is in the main rotor disk and many observer positions below the helicopter are shielded by the fuselage. The tail rotor, which typically operates with a BPF that is significantly higher than the main rotor, also generates noise that is subject to scattering and shielding by the fuselage but it is less affected because of its position at the extreme rear of the helicopter.

Atalla and Glegg $[107,108]$ used a ray-acoustics approach to study the fuselage scattering of helicopter rotor noise. Their method was a combination of classical geometrical acoustics and the paraxial ray approximation with BVI modeled by a rotating point source. The primary effect of scattering of rotor noise was to cause a secondary acoustic pulse of smaller amplitude than the direct pulse - the phase and amplitude of which were highly dependent upon observer position. Any such additions to the acoustic signal clearly will modify both the amplitude and directivity of the acoustic field at the measurement locations. Atalla and Glegg also observed another important effect for the case of a simplified tiltrotor geometry (see figure 22). In this case, an additional impulse in the acoustic pressure signal was observed at some locations because the fuselage blocks the acoustic rays from the source during part of the motion of the source but not all of it. As the source appears from behind the body, the acoustic signal is heard and creates the extra impulse shown in figure 23 . The impulse shown in figure 23 is exaggerated because the ray-acoustic method does not account for diffraction, nevertheless, this exam- 
ple emphasizes an important modification of the acoustic field-neglected in a free-field noise computation - is possible. Atalla and Glegg's work does not clearly show whether or not these effects will be strong for a real helicopter.

The ray acoustics approach to the scattering problem has the drawback that the method fails in the shadow zone where diffraction effects are important. Furthermore, the method is somewhat complicated to use when the scattering body does not have a simple geometry. To overcome these limitations, Chung and Morris $[109,110]$ developed a computational approach they have called the impedance mismatch method (IMM). The linear Euler equations are solved for wave propagation using high-accuracy finite difference algorithms. The IMM simplifies the implementation of a solid wall boundary condition by setting the acoustic impedance of the fluid to a different value inside of the scattering body. This impedance difference results in reflected and transmitted waves with appropriate amplitudes. The primary advantage of this approach is that a simple Cartesian grid can be used to model the computational domain even for complex geometries. Thus high-order finite difference schemes, suitable for computational aeroacoustics (CAA), can be readily used.

Laik and Morris [111] modified the IMM such that the acoustic field is separated into incident and scattered fields. This separation is valuable because the incident field can be obtained from an analytical solution or a numerical prediction scheme such as WOPWOP without propagating the incident field throughout the finite-difference grid. Agarwal and Morris [112] used the modified IMM methodology to compute the acoustic near-field around a realistic rotorcraft fuselage model. The directivity of the acoustic field was changed significantly by the presence of the fuselage. Although Morris and his coworkers have yet to use an incident field from a numerical prediction (such as WOPWOP), this is needed to quantify the amount of scattering and diffraction present in wind tunnel and flight test data.

\subsubsection{Maneuvering Rotorcraft}

The problem of the prediction of noise for a rotorcraft in a maneuver, poses a daunting and largely unaddressed challenge. In both civil and military operations, maneuvering flight is essential for a rotorcraft to perform its intended mission. Nevertheless, current rotor-noise-prediction methods have not until recently addressed even the simplest maneuvers, such as accelerating and decelerating flight in rectilinear motion. Aircraft motions - such as pitch, roll, or yaw motions - cause significant time-dependent shifts in the noise directivity. Furthermore, transient maneuvers can generate a substantial increase in the noise radiation due to both kinematic and aerodynamic effects. Recently Gopalan et al. [113] have developed a method to predict the first-order effects of acceleration (deceleration) parallel to the flight path. Janakiram and 
Khan [114] have performed a detailed state-of-the-art prediction and validation for a helicopter in descending and decelerating flight. In both of these works, quasisteady analyses (i.e., predictions for a series of steady trim states made at several points along the flight path) were used for flight conditions where the aircraft state varied along a straight flight path. Aircraft acceleration was included in the trim computation, but it was not represented in the noise prediction directly.

Recently, Brentner and Jones [115] provided an initial demonstration of the importance of both steady and transient maneuvers for a notional helicopter. As an example of a steady maneuver, they compared the noise of level and turning flight on a plane $100 \mathrm{ft}$ below the helicopter for a moderate forward speed of 120 knots. The turn radius and turn rate were $1160.5 \mathrm{ft}$ and 10 $\mathrm{deg} / \mathrm{sec}$, respectively, for both the right and left turns. The aircraft attitude and blade loads ${ }^{18}$ were computed by CAMRAD 2 [116]. Figure 24 shows a comparison of the OASPL contours for level and turning flight. The small circle in the center of the contour plot represents the projection of the main rotor disk onto the measurement plane. It is interesting that the turning flight peak OASPL levels are as much as 10 decibels $(\mathrm{dB})$ higher than the level flight case. This is a result of the significant increase in rotor loading required for turning flight. Although loading is nearly the same for left and right turns, there is a significant difference in the OASPL directivity of the right and left turns. This difference is related to both the tail rotor thrust and rotation direction of the main rotor.

Brentner et al. $[55,115,117]$ also demonstrated the potential for increased noise in a transient maneuver. They considered a notional helicopter originally in a $3 \mathrm{deg}$. descent that is arrested by a momentary increase in collective pitch. The collective pitch used in the maneuver is shown in figure 25 and the resulting aircraft position is shown in figure 26. This arrested descent maneuver (also known as a pull up) is a fairly aggressive maneuver - the peak deceleration is $1.7 \mathrm{~g}$. During the maneuver, blade motion and loading are not periodic and are different for each blade. Figure 27 show the acoustic pressure time history for two observer locations below the helicopter. Although the peak blade loading is nearly doubled, the acoustic pressure is increased by 3 to 5 times depending upon the observer location. The amount of increase in noise is highly dependent upon the observer position. Brentner et al. [117] computed the directivity of the noise during the arrested descent maneuver, shown in figure 28. In this figure, contours of OASPL for both the steady $3 \mathrm{deg}$. descent case and the transient arrested descent are compared at several times. At the beginning and end of the maneuver the noise levels are essentially the same, but during the maneuver, the OASPL is increased by $12 \mathrm{~dB}$ at some

\footnotetext{
${ }^{18}$ Blade-vortex interactions were not fully resolved in the CAMRAD 2 computations.
} 
locations in the field. This tremendous increase in noise levels is due to both the temporary increase in blade loading and other transient effects. More details about these computations can be found in refs. 55, 115, 117-119. A more thorough analysis is needed to determine whether or not such increases are likely for specific real maneuvers - especially if blade-vortex interaction is involved.

These examples show that the noise of a rotorcraft in maneuvering flight can be considerably different than that for the same rotorcraft in a steady flight condition. Nevertheless, these are early computations and much more work needs to be done. In the present computations, blade-vortex interactions have been inadequately resolved. Given the difficulty of steady free-wake computations, the maneuver wake problem will likely be even more difficult. Another problem that is an issue for maneuvering flight is that the time scale for a maneuver calculation is much longer than that for steady flight. This difference can be computationally quite significant.

\section{Concluding Remarks}

In the last several years significant progress has been made in the development of effective noise prediction models for helicopter main rotor noise. Advances have been made on all fronts: BVI noise is well understood and can be predicted, but aerodynamic loads computations are challenging and more understanding of the rotor wake and tip vortices is needed. HSI noise prediction has reached and advanced state because there was an immediate need for an efficient HSI noise prediction tool. New advances have been made in broadband noise prediction and it appears that even more rigorous methods are on the horizon. The $\mathrm{FW}-\mathrm{H}$ equation has proven to be a fruitful source of integral formulations and the desire to avoid the computation of the quadrupole noise altogether has lead to the use of the FW-H equation on permeable surfaces. The permeable-surface FW-H equation actually embodies all of HSI prediction methods because the full formulation still includes the quadrupole outside of the integration surface. It has also been demonstrated that the Kirchhoff formulation, while suitable for acoustic problems, can be unreliable for aeroacoustics. Fortunately the FW-H equation is equally efficient and much more robust.

Although this article has not dealt directly with rotorcraft aerodynamic computations, it should be pointed out that the acoustic formulations discussed require highly accurate solutions - both spatially and temporally - as input data. The current aerodynamic state-of-the-art for even simple rotor configurations is at best "relatively good." The aerodynamic calculations required

for HSI noise in forward flight are extremely challenging. An accurate, first- 
principles, blade-vortex interaction computation is still beyond the ability of present day CFD, but many researchers are attacking the problem. Full vehicle and maneuver noise computations may be even more challenging. Nevertheless, progress is being made and the acoustic propagation theory and codes will be up to the task.

\section{References}

[1] K. S. Brentner, F. Farassat, Helicopter noise prediction: The current status and future direction, Journal of Sound and Vibration 170 (5) (1994) 79-96.

[2] L. Gutin, On the sound of a rotating airscrew, Zhurnal Technicheskoi Fiziki 6 (1936) 889-909, translated as NACA TM 1192, 1948.

[3] A. F. Deming, Noise from propellors with symmetrical sections at zero blade angle, ii., NACA TM 679 (1938).

[4] W. Ernsthausen, The source of propeller noise, Luftfahrtforschung 8 (1936) 433-440, translated as NACA TM 825, 1937.

[5] I. E. Garrick, C. E. Watkins, A theoretical study of the effect of forward speed on the free-space sound-pressure field around propellors, NACA Report 1198 (1954).

[6] M. V. Lowson, The sound field of singularities in motion, Proceedings of the Royal Society A286 (1965) 559-572.

[7] S. E. Wright, Sound radiation from a lifting rotor generated by asymmetric disc loading, Journal of Sound and Vibration 9 (1969) 223-240.

[8] M. V. Lowson, J. B. Ollerhead, A theoretical study of helicopter rotor noise, Journal of Sound and Vibration 9 (1969) 197-222.

[9] J. W. Leverton, Helicopter noise, in: AGARD Conference Proceedings, Vol. 31, 1968.

[10] M. V. Lowson, Helicopter noise: analysis-prediction and methods of reduction, AGARD Report LS-63 (1973).

[11] J. E. Ffowcs Williams, D. L. Hawkings, Sound generated by turbulence and surfaces in arbitrary motion, Philosophical Transactions of the Royal Society A264 (1151) (1969) 321-342.

[12] M. J. Lighthill, On sound generated aerodynamically, I: general theory, Proceedings of the Royal Society A211 (1952) 564-587.

[13] D. L. Hawkings, M. V. Lowson, Theory of open supersonic rotor noise, Journal of Sound and Vibration 36 (1974) 1-20. 
[14] F. Farassat, Theory of noise generation from moving bodies with an application to helicopter rotors, NASA TR R-451 (1975).

[15] F. Farassat, R. J. Pegg, D. A. Hilton, Thickness noise of helicopter rotors at high tip speeds, AIAA Paper 75-453 (1975).

[16] F. Farassat, Linear acoustic formulas for calculation of rotating blade noise, AIAA Journal 19 (1981) 1122-1130.

[17] F. H. Schmitz, Y. H. Yu, Theoretical modeling of high-speed helicopter impulsive noise, Proceedings of the Third European Rotorcraft and Powered Lift Aircraft Forum, paper 54 (1977).

[18] D. B. Hanson, M. R. Fink, The importance of quadrupole sources in prediction of transonic tip speed propeller noise, Journal of Sound and Vibration 62 (1) (1979) 19-38.

[19] Y. H. Yu, F. X. Caradonna, F. H. Schmitz, The influence of the transonic flow field on high-speed helicopter impulsive noise, Fourth European Rotorcraft and Powered Lift Aircraft Forum, paper 58 (1978).

[20] A. R. George, Helicopter noise - state of the art, AIAA Paper 77-1337 (1977).

[21] F. H. Schmitz, Y. H. Yu, Helicopter impulsive noise: theoretical and experimental status, NASA TM 84390 (1983).

[22] O. Childress, Jr., The NASA/AHS rotorcraft noise reduction program., Vertiflite 34 (1988) 18-22.

[23] F. H. Schmitz, D. A. Boxwell, W. R. Splettstoesser, K. J. Schultz, Model-rotor high-speed impulsive noise: Full-scale comparisons and parametric variations, Vertica 8 (4) (1984) 395-422.

[24] W. R. Splettstoesser, K. J. Schultz, D. A. Boxwell, F. H. Schmitz, Helicopter model rotor-blade vortex interaction impulsive noise: scalability and parametric variations, NASA TM 86007 (1984).

[25] R. M. Martin, W. R. Splettstoesser, Blade-vortex interaction acoustic results from a forty percent model rotor in the DNW, Journal of the American Helicopter Society 33 (1988) 37-46.

[26] T. F. Brooks, M. A. Marcolini, D. S. Pope, Main rotor broadband noise study in the DNW, Journal of the American Helicopter Society 34 (1989) 3-12.

[27] S. R. Liu, M. A. Marcolini, The acoustic results of a United Technologies scale model helicopter rotor tested at DNW, American Helicopter Society 46th Annual Forum (1990).

[28] F. Farassat, Introduction to generalized functions with applications in aerodynamics and aeroacoustics, NASA TP 3428, see corrected version, April 1996. (May 1994).

[29] F. Farassat, The Kirchhoff formulas for moving surfaces in aeroacoustics the subsonic and supersonic cases, NASA TM 110285 (Sep. 1996). 
[30] I. M. Gel'fand, G. E. Shilov, Generalized Functions: Properties and Operations, Vol. 1, Academic Press, Inc., 11 Fifth Avenue, New York 3, New York, 1964, translated by Eugene Saletan, Department of Physics, Northeastern University, Boston, Massachuesetts.

[31] D. S. Jones, The Theory of Generalised Functions, 2nd Edition, Cambridge University Press, Cambridge, England, 1982.

[32] R. P. Kanwal, Generalized Functions - Theory and Technique, 2nd Edition, Birkhauser, Boston, 1998.

[33] D. J. Struik, Lectures on Classical Differential Geometry, Dover Publications, Inc., New York, 1988, this is an unabridged and unaltered republication of the second edition (1961) of the work first published in 1950 by the AddisonWesley Publishing Company, Inc, Reading, Massachusetts.

[34] R. Aris, Vectors, Tensors, and the Basic Equations of Fluid Mechanics, Dover Publications, Inc., New York, 1989, unabridged republication of the edition published by Prentice-Hall, Inc, Englewood Cliffs, N.J., 1962.

[35] A. J. McConnell, Applications of Tensor Analysis, Dover Publications, Inc., New York, 1957, unaltered, unabridged republication of the work originally published by the Blackie Company in 1931 under the title Applications of the Absolute Differential Calculus.

[36] M. P. Isom, The theory of sound radiated by a hovering transonic helicopter blade, Poly-AE/AM No. 75-4, Polytechnic Institute of New York (1975).

[37] F. Farassat, Extension of Isom's thickness noise formula to the near field, Journal of Sound and Vibration 67 (2) (1979) 280-281.

[38] F. Farassat, R. M. Martin, Note on the tip noise of rotating blades, Journal of Sound and Vibration 86 (3) (1983) 449-453.

[39] J. E. Ffowcs Williams, Sound sources in aerodynamics - fact and fiction, AIAA Journal 20 (3) (1982) 307-315.

[40] F. Farassat, Y.-J. Lee, H. Tadghighi, R. Holz, High-speed helicopter rotor noise - shock waves as a potent source of sound, American Helicopter Society and Royal Aeronautical Society International Technical Specialists Meeting: Rotorcraft Acoustics and Rotor Fluid Dynamics (Oct. 1991).

[41] F. Farassat, K. S. Brentner, The uses and abuses of the acoustic analogy in helicopter rotor noise prediction, Journal of the American Helicopter Society 33 (1) (1988) 29-36.

[42] F. Farassat, M. Farris, The mean curvature of the influence surface of wave equation with sources on a moving surface, Mathematical Methods in the Applied Sciences (1999) 1485-1503.

[43] F. Farassat, G. P. Succi, The prediction of helicopter discrete frequency noise, Vertica 7 (4) (1983) 309-320. 
[44] K. S. Brentner, Prediction of helicopter discrete frequency rotor noise - a computer program incorporating realistic blade motions and advanced acoustic formulation, NASA TM 87721 (Oct. 1986).

[45] K. S. Brentner, An efficient and robust method for predicting helicopter rotor high-speed impulsive noise, Journal of Sound and Vibration 203 (1).

[46] Y. Xue, A. S. Lyrintzis, Rotating Kirchhoff method for three-dimensional transonic blade-vortex interaction hover noise, AIAA Journal 32 (7) (1994) 1350-1359.

[47] Y. Özyörük, L. N. Long, A Navier-Stokes/Kirchhoff method for noise radiation from ducted fans, AIAA Paper 94-0462 (1994).

[48] W. H. Press, B. P. Flannery, S. A. Teukolsky, W. T. Vetterlin, Numerical Recipes: The Art of Scientific Computing, Cambridge University Press, 1986.

[49] K. R. Meadows, H. L. Atkins, Towards a highly accurate implementation of the Kirchhoff approach for computational aeroacoustics, Journal of Computational Acoustics 4 (2) (1996) 225-241.

[50] F. Farassat, M. H. Dunn, S. Padula, Advanced turboprop noise prediction based on recent theoretical results, Journal of Sound and Vibration 119 (1987) $53-79$.

[51] M. H. Dunn, G. M. Tarkenton, Computational methods in the prediction of advanced subsonic and supersonic propeller induced noise-ASSPIN user's manual, NASA CR 4434 (Apr. 1992).

[52] K. S. Brentner, P. C. Holland, An efficient and robust method for computing quadrupole noise, Journal of the American Helicopter Society 42 (2) (1997) $172-181$.

[53] Advanced Rotocraft Technology, Inc., Kichhoff code - a versatile CAA tool, NASA SBIR Phase I Final Report, contract NAS1-20366 (Jun. 1995).

[54] J. G. Leishman, Aeroacoustics of 2D and 3D blade-vortex interaction using the indicial method, American Helicopter Society 52nd Annual Forum Proceedings (Jun. 1996).

[55] K. S. Brentner, G. A. Brès, G. Perez, H. E. Jones, Maneuvering rotorcraft noise prediction: A new code for a new problem, AHS Aerodynamics, Acoustics, and Test Evaluation Specialist Meeting (Jan. 2002).

[56] F. Farassat, T. J. Brown, A new capability for predicting helicopter rotor and propeller noise including the effect of forward motion, NASA TM X-74037 (Jun. 1977).

[57] D. B. Hanson, Near field noise of high tip speed propellers in forward flight, AIAA Paper 76-565 (1976). 
[58] F. Farassat, M. K. Myers, Line source singularity in the wave equation and its removal by quadrupole sources - a supersonic propeller noise problem, in: J. E. Ffowcs Williams, D. Lee, A. D. Pierce (Eds.), Structural Acoustics, Scattering, and Propagation, World Scientific, Singapore, 1994, pp. 29-43.

[59] K. S. Brentner, A new algorithm for computing acoustic integrals, in: Proceedings of the IMACS 14th World Congress on Computational and Applied Mathematics, Vol. 2, 1994, pp. 592-595.

[60] W. E. Lorensen, H. E. Cline, Marching cubes: A high resolution 3D surface construction algorithm, Computer Graphics 21 (4) (1987) 163-169.

[61] F. Farassat, K. S. Brentner, Supersonic quadrupole noise theory for high-speed helicopter rotors, Journal of Sound and Vibration 218 (3) (1998) 481-500.

[62] S. Ianniello, Quadrupole noise predictions through the FW-H equation, AIAA Journal 37 (9) (1999) 1048-1054.

[63] S. Ianniello, Aerocoustic analysis of high tip-speed rotating blades, Aerospace Science and Technology 5 (3) (2001) 179-192.

[64] S. Ianniello, Algorithm to integrate the Ffowcs Williams-Hawings equation on supersonic rotating domain, AIAA Journal 37 (9) (1999) 1040-1047.

[65] T. W. Purcell, CFD and transonic helicopter sound, Fourteenth European Rotorcraft Forum, paper 2 (1988).

[66] J. A. Visintainer, M. A. Marcolini, C. L. Burley, S. R. Liu, Acoustic predictions using measured pressures from a model rotor in the DNW, Journal of the American Helicopter Society 38 (3) (1993) 35-44.

[67] C. L. Burley, H. Tadghighi, Importance of high accuracy blade motion and airloads predictions in acoustic analysis, American Helicopter Society 50th Annual Forum (1994).

[68] F. H. Schmitz, Chapter 2 rotor noise, in: H. H. Hubbard (Ed.), Aeroacoustics of Flight Vehicles: Theory and Practice, Vol. 1: Noise Sources, 1991, pp. 65-149.

[69] M. V. Lowson, Progress towards quieter civil helicopters, Aeronautical Journal (1992) 209-223.

[70] M. V. Lowson, Focusing on helicopter BVI noise, Journal of Sound and Vibration 190 (3) (1996) 477-494.

[71] Y. H. Yu, Rotor blade-vortex interaction noise, Progress in Aerospace Sciences 36 (2) (2000) 97-115.

[72] T. F. Brooks, D. D. Boyd, Jr., C. L. Burley, J. R. Jolly, Jr., Aeroacoustic codes for rotor harmonic noise and BVI noise-CAMRAD.Mod1/HIRES, AIAA Paper 96-1735 (May 1996).

[73] C. L. Burley, M. A. Marcolini, T. F. Brooks, A. G. Brand, D. A. Conner, Tiltrotor aeroacoustic code (TRAC) prediction and comparison with measurements, American Helicopter Society 52nd Annual Forum (1996). 
[74] W. Johnson, A comprehensive analytical model of rotor aerodynamics and dynamics, part i: Analytical development, NASA TM 81182 (Jun. 1980).

[75] W. R. Splettstoesser, R. Kube, U. Seelhorst, W. Wagner, A. Boutier, F. Micheli, E. Mercker, K. Pengel, Key results from a higher harmonic control aeroacoustic rotor test (HART) in the German-Dutch wind tunnel, TwentyFirst European Rotorcraft Forum (1995).

[76] M. A. Marconlini, D. A. Conner, J. T. Brieger, L. E. Becker, C. D. Smith, Noise characteristics of a model tiltrotor, American Helicopter Society 51st Annual Forum (1995).

[77] R. K. Singh, J. D. Baeder, Transonic effects on acoustics of blade-vortex interaction, AIAA Paper 2000-1921 (Jun. 2000).

[78] R. K. Singh, Transonic effects on aerodynamics and acoustics of blade-vortex interaction, Ph.D. dissertation, University of Maryland, College Park, MD (1999).

[79] F. Caradonna, C. Kitaplioglu, M. McCluer, J. Baeder, J. G. Leishman, C. Berizin, J. Visintainer, J. Bridgeman, C. Burley, R. Epstien, A. Lyrintzis, E. Koutsavdis, G. Rahier, Y. Delrieux, J. Rule, D. Bliss, Methods for the predicition of blade-vortex interaction noise, Journal of the American Helicopter Society 45 (4) (2000) 303-317.

[80] C. Kitaplioglu, F. Caradonna, Aerodynamics and acoustics of blade-vortex interaction using and independently generated vortex, American Helicopter Society Aeromechanics Specialists Conference (Jan. 1994).

[81] Y. H. Yu, B. Gmelin, H. Heller, J. J. Philippe, E. Mercker, J. S. Preisser, HHC aeroacoustic rotor test at the DNW the joint German/French/US project, 20th European Rotorcraft Forum (1994).

[82] R. Kube, W. R. Splettstoesser, W. Wagner, U. Seelhorst, Y. H. Yu, A. Boutier, F. Micheli, W. Mercker, Initial results from the higher harmonic control aeroacoustic rotor test (HART) in the German-Dutch wind tunnel, 75th Fluid Dynamics Panel Symposium on Aerodynamics and Aeroacoustics of Rotorcraft (1994).

[83] W. R. Splettstoesser, R. Kube, U. Seelhorst, W. Wagner, A. Boutier, F. Micheli, E. Mercker, K. Pengel, Key results from a higher harmonic control aeroacoustic rotor test (HART) in the German-Dutch wind tunnel, 21st European Rotorcraft Forum (1995).

[84] Y. H. Yu, C. Tung, B. van der Wall, Heinz-Jürgen, C. L. Burley, T. F. Brooks, P. Beaumier, Y. Delrieux, E. Mercker, K. Pengel, The HART-II test: Rotor wakes and aeroacoustics with higher-harmonic pitch control (HHC) inputs - the joint German/French/Dutch/US project, American Helicopter Society 58th Annual Forum Proceedings (2002).

[85] T. F. Brooks, C. L. Burley, Rotor broadband noise predicition with comparison to model data, AIAA Paper 2001-2210 (May 2001). 
[86] T. F. Brooks, C. L. Burley, Blade wake interaction noise for a model main rotor, AHS Technical Specialists' Meeting for Rotorcraft Acoustics and Aerodynamics, Williamsburg, Virginia (Oct. 1997).

[87] R. K. Amiet, Acoustic radiation from an airfoil in a turbulent stream, Journal of Sound and Vibration 41 (1975) 407-420.

[88] J. Casper, F. Farassat, Broadband noise predictions based on a new aeroacoustic formulation, AIAA Paper 2002-0802 (Jan. 2002).

[89] M. S. Howe, Trailing edge noise at low Mach numbers, Journal of Sound and Vibration 225 (2) (1999) 211-238.

[90] M. S. Howe, Trailing edge noise at low Mach numbers, part 2: Attached and separated edge flows, Journal of Sound and Vibration 234 (5) (2000) 761-775.

[91] B. A. Singer, K. S. Brentner, D. P. Lockard, G. M. Lilley, Simulation of acoustic scattering from a trailing edge, Journal of Sound and Vibration 230 (3) (2000) $541-560$.

[92] C. L. Burley, T. F. Brooks, B. D. Charles, M. McCluer, Tiltrotor aeroacoustic code (TRAC) prediction assessment and initial comparisons with TRAM test data, Twenty-Fifth European Rotorcraft Forum, paper B3 (Sep. 1999).

[93] C. L. Burley, T. F. Brooks, M. A. Marcolini, A. G. Brand, D. A. Conner, Tiltrotor aeroacoustic code (TRAC) predictions and comparisons with measurements, Journal of the American Helicopter Society 45 (2) (2000) 8089.

[94] J. E. Ffowcs Williams, L. H. Hall, Aerodynamic sound generation by turbulent flow in the vicinity of a scattering half-plane, Journal of Fluid Mechanics 40 (1970) 657-670.

[95] M. Wang, Aerodynamic sound of flow past an airfoil, Annual Research Briefs - 1995, Center for Turbulence Research, Stanford University/NASA Ames Research Center, 1995, pp. 257-271.

[96] U. Piomelli, C. L. Streett, S. Sarkar, On the computation of sound by largeeddy simulations, Journal of Engineering Mathematics 32 (1997) 217-236.

[97] F. Souliez, L. N. Long, P. J. Morris, A. Sharma, Landing gear aerodynamic noise prediction using unstructured grids, International Journal of Aeroacoustics 1 (2) (2002) 115-135.

[98] C. K. W. Tam, J. C. Webb, Dispersion-relation-preserving finite difference schemes for computational aeroacoustics, Journal of Computational Physics 107 (1993) 262-281.

[99] A. A. Oberai, F. Roknaldin, T. J. R. Hughes, Computational procedures for determining structural-acoustic response due to hydrodynamic sources, Computational Methods in Applied Mechanical Engineering 190 (2000) 345361. 
[100] D. G. Crighton, A. P. Dowling, J. E. Ffowcs Williams, M. Heckl, F. G. Leppington, Modern Methods in Analytical Acoustics: Lecture Notes, Springer-Verlag, London, 1992.

[101] P. di Francescantonio, A new boundary integral formulation for the prediction of sound radiation, Journal of Sound and Vibration 202 (4) (1997) 491-509.

[102] K. S. Brentner, F. Farassat, An analytical comparison of the acoustic analogy and Kirchhoff formulation for moving surfaces, AIAA Journal 36 (8) (1998) $1379-1386$.

[103] A. R. Pilon, A. S. Lyrintzis, Integral methods for computational aeroacoustics, AIAA Paper 97-0020 (Jan. 1997).

[104] R. C. Strawn, E. P. N. Duque, J. Ahmad, Rotorcraft aeroacoustics computations with overset-grid CFD methods, Journal of the American Helicopter Society 44 (2) (1999) 132-140.

[105] J. Prieur, G. Rahier, Comparison of Ffowcs Williams-Hawkings and Kirchhoff rotor noise calculations, AIAA Paper 98-2376 (1998).

[106] F. Farassat, M. K. Myers, Extension of Kirchhoff's formula to radiation from moving surfaces, Journal of Sound and Vibration 123 (3) (1988) 451-461.

[107] N. Atalla, S. A. L. Glegg, Ray-acoustics approach to fuselage scattering of rotor noise, Journal of the American Helicopter Society 38 (3) (1993) 56-63.

[108] N. Atalla, S. A. L. Glegg, A geometrical acoustics approach for calculating the effects of flow on acoustic scattering, Journal of Sound and Vibration 171 (5) (1994) 681-694.

[109] C. Chung, Wave propagation and scattering in computational aeroacoustics, Ph.D. dissertation, Dept. of Aerospace Engineering, Pennsylvania State University, University Park, PA (1995).

[110] C. Chung, P. J. Morris, Acoustic scattering from two- and three-dimensional bodies, Journal of Computational Acoustics 6 (3) (1998) 357-375.

[111] O. A. Laik, P. J. Morris, Direct simulation of acoustic scattering by two- and three-dimensional bodies, Journal of Aircraft 37 (1) (2000) 68-75.

[112] A. Agarwal, P. J. Morris, Direct simulation of acoustic scattering by a rotorcraft fuselage, AIAA Paper 2000-2030 (Jun. 2000).

[113] G. Gopalan, F. H. Schmitz, B. W.-C. Sim, Flight path management and control methodology to reduce helicopter blade-vortex interaction (BVI) noise, American Helicopter Society Vertical Lift Design Conference (Jan. 2000).

[114] R. D. Janakiram, H. Khan, Prediction and validation of helicopter descent flyover noise, AHS International 56th Annual Forum Proceedings (May 2000).

[115] K. S. Brentner, H. E. Jones, Noise prediction for maneuvering rotorcraft, AIAA Paper 2000-2031 (2000). 
[116] W. Johnson, Comprehensive analytical model of rotorcraft aerodynamics and dynamics, Volumes I - VII, Johnson Aeronautics (1998).

[117] K. S. Brentner, G. Perez, G. A. Brès, H. E. Jones, Toward a better understanding of maneuvering rotorcraft noise, American Helicopter Society 58th Annual Forum Proceedings (Jun. 2002).

[118] G. A. Brès, Modeling of the noise of arbitrary maneuvering rotorcraft: Analysis and implementation of the PSU-WOPWOP noise prediction code, MS thesis, Dept. of Aerospace Engineering, The Pennsylvania State University, University Park, PA (May 2002).

[119] G. Perez, Investigation of the influence of maneuver on rotorcraft noise, MS thesis, Dept. of Aerospace Engineering, The Pennsylvania State University, University Park, PA (May 2002). 


\section{List of Figures}

1 A schematic of the complicated aeromechanical environment in which a helicopter rotor operates.

2 Typical direction of primary radiation for various rotor noise sources.

3 Nondimensional pressure on a meridian line of a spherical Kirchhoff integration surface plotted at a function of the source time $\tau$ (i.e., a single time step from the CFD solution) and as a function of the retarded time $\tau *$, from [49].

4 Comparison of predicted solution with and without an adaptive quadrature algorithm. (a) No adaptation, mid-panel quadrature. (b) Adaptive quadrature. (Note that considerable panel size variation occurred in this computation.)

5 Schematic of collapsing sphere, rotor blade and their intersection.

6 Schematic of logical grid, marching cube and triangular piece of surface in marching cube.

$7 \quad$ Far-field approximation of the collapsing sphere. Although not shown, the observer is three rotor radii to the right of the rotor blade.

8 Comparison of WOPWOP $+(-)$ and WOPWOP2+ (- - ) predicted acoustic pressure with experimental data [65] (a) for hovering model UH-1H rotor. Quadrupole grid in WOPWOP + prediction extended almost to sonic circle and in WOPWOP2+ predictions extended $1.86 R$ beyond the rotor tip.

9 Comparison of predicted acoustic pressure (-) [62] with experimental data [65] () for hovering model UH-1H rotor.

10 Contemporary design, four-blade model rotor operating in forward flight; $\mu=0.32$ and $M_{A T}=0.933$. experimental data; (-) predicted acoustic pressure; $(---$ )quadrupole component of predicted acoustic pressure.

11 Schematic of blade-vortex interaction. 
12 Comparison of measured and predicted noise for a four-bladed Sikorsky model rotor. The microphone location for this comparison was nominally $25^{\circ}$ below the rotor plane on the advancing side, $\psi=150^{\circ}, 1.5$ rotor radii from the rotor hub. This is a descent condition, $\mu=.152$ and $C_{T} / \sigma=.07$, which corresponds to figure 3 in ref. [66]. (a) Measured time history; (b) predicted time history; (c) spectral comparison, measured; $\diamond$, predicted.

13 Schematic of the TiltRotor Aeroacoustic Codes (TRAC) developed at the NASA Langley Research Center for helicopter and tiltrotor noise prediction [72].

14 A comparison of measured and predicted acoustic pressure time histories for the BO-105 HART model. For this case, $\mu=0.15, C_{T}=0.0044, M_{H}=0.64$ and the shaft angle $\alpha_{s}=5.3 \mathrm{deg}$. The observer is located $2.4 \mathrm{~m}$ below and $1.61 \mathrm{~m}$ right of the hub under the advancing side of the rotor. (This is figure 20(a) in ref. [72].) (a) experiment (b) prediction

15 Comparison of sound pressure measurement and predictions on the advancing side of a JVX tiltrotor model operating in hover. For this case, $\mu=0.17, C_{T}=0.00984, M_{H}=0.64$ and the shaft angle $\alpha_{s}=3.15 \mathrm{deg}$. The observer is located 1.512 $\mathrm{m}$ below, $1.372 \mathrm{~m}$ forward and $0.711 \mathrm{~m}$ right of the rotor hub. (This is a combination of figures 27 and 32 in ref. [72]. See refs. [72] and [73] for more details, including wind tunnel flow corrections.) (a) measured, (b) predicted - with roll-up model, (c) predicted-no roll-up model.

16 An illustration of flow conditions producing blade self noise (from Brooks and Burley [85]).

17 Comparison of noise components predicted by the FW$\mathrm{H} / \mathrm{RKIR}$ and WOPWOP + codes for a hovering UH-1H model rotor $\left(M_{H}=0.88\right.$, inplane observer $3.4 R$ from rotor hub).

18 Concentric cylindrical integration surfaces used for noise computation for a hovering rotor.

19 Predicted acoustic pressure for various integration surface locations for an observer located $3.4 R$ from a UH-1H model rotor hovering at $M_{H}=0.88$. The experimental data ( $\square$ ) is from ref. 65. (a) FW-H prediction; (b) Kirchhoff prediction 
20 Vorticity field computed from CFD. FW-H integration surfaces are at $r=0.5 D, r=1.5 D, r=2.5 D$ and $r=5.1 D$

21 Acoustic signals computed for various integration surfaces that correspond to those indicated in Figure 20.

22 Geometry of simple tiltrotor model which results in an additional impulse in the acoustic pressure time history [107].

23 Steady loading noise from a rotating steady point force near a rigid body (idealized tiltrotor model). (a) free-field, (b) near a rigid box. The source Mach number is $M=0.7$ and the observer is in far-field $45 \mathrm{deg}$. below and on the right side of the tiltrotor fuselage [107].

24 Comparison of main-rotor noise overall sound pressure levels in level and turning flight [115]. (a) left turn, (b) level flight, (c) right turn; The flight direction is toward the top of the figure.

25 Collective pitch control during arrested descent maneuver [55].

26 Aircraft position during arrested descent maneuver [55]. The aircraft was at $\mathbf{x}=(0 ., 0 ., 0$. $)$ at $t=0 \mathrm{~s}$.

27 Comparison of acoustic pressure time history for a three-degree steady descent (- - - ) and an arrested descent maneuver ( - ) . (a) observer located $100 \mathrm{ft}$ below the helicopter at $t=0$ (from ref. [55])(b) observer located at $100 \mathrm{ft}$ below, 100 $\mathrm{ft}$ to the right and $100 \mathrm{ft}$ forward of the helicopter at $t=0$ (from ref. [117])

28 Comparison of OASPL contours for the three-degree steady descent to the arrested descent maneuver as a function of time [117]. The circle is the projection of the main-rotor disk on the measurement plane. 


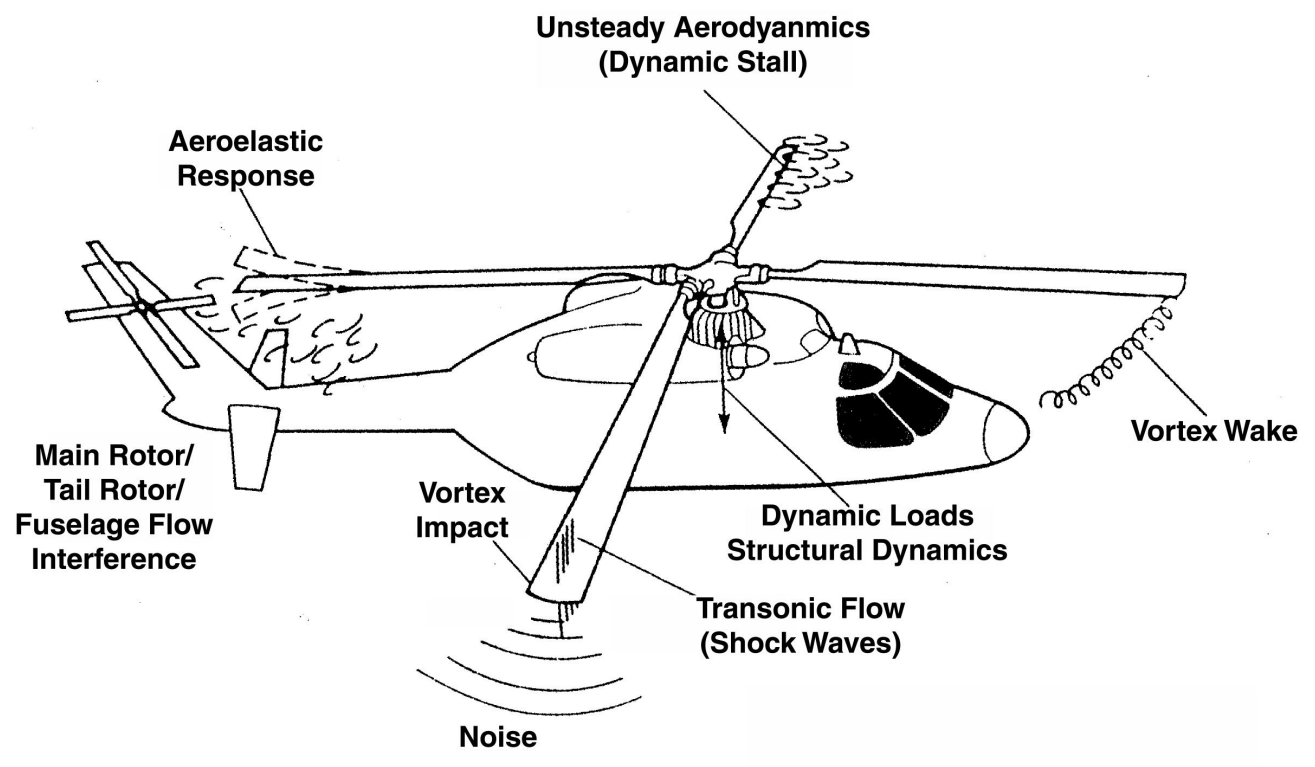

Fig. 1. A schematic of the complicated aeromechanical environment in which a helicopter rotor operates. 

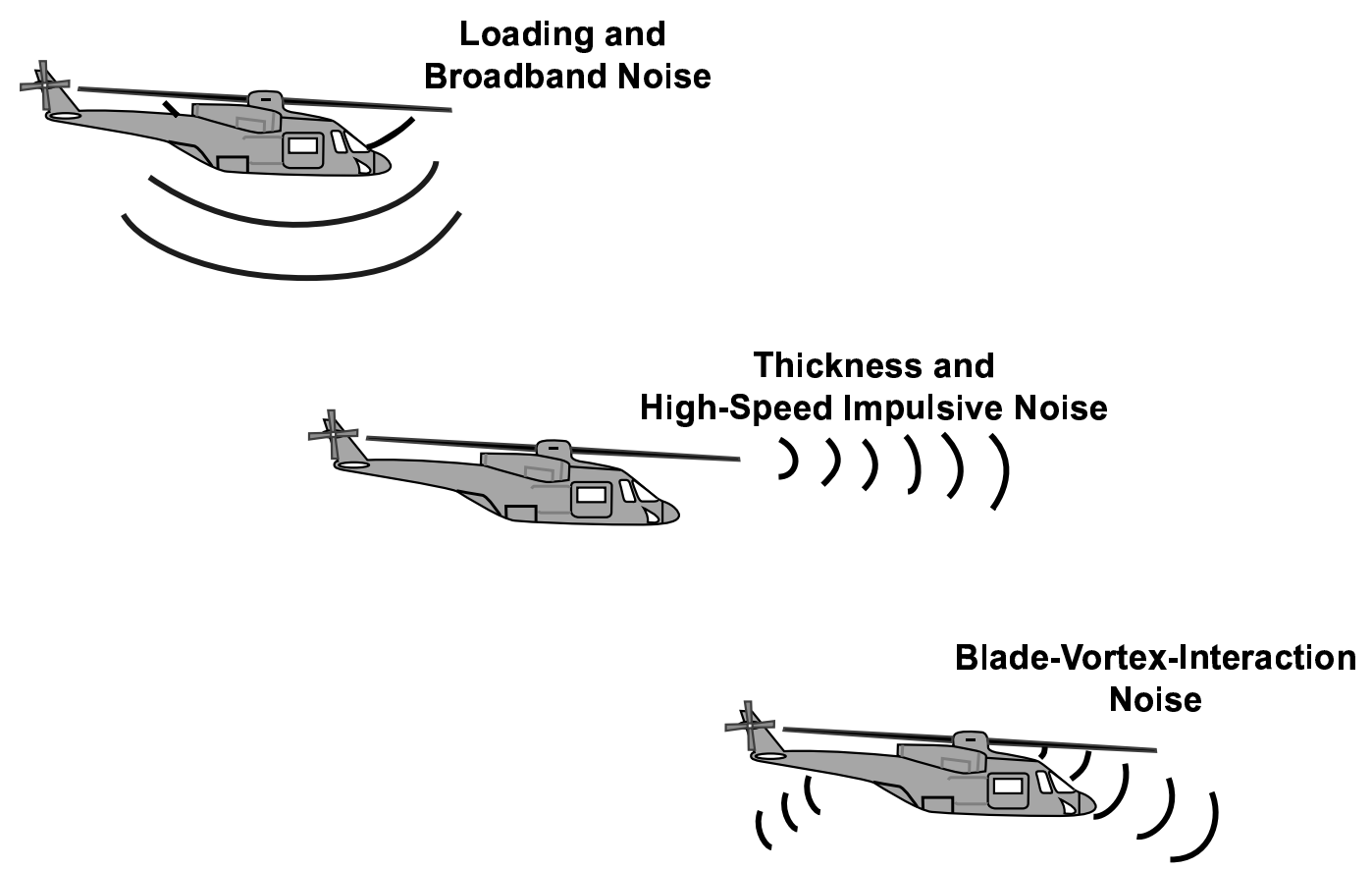

Fig. 2. Typical direction of primary radiation for various rotor noise sources. 


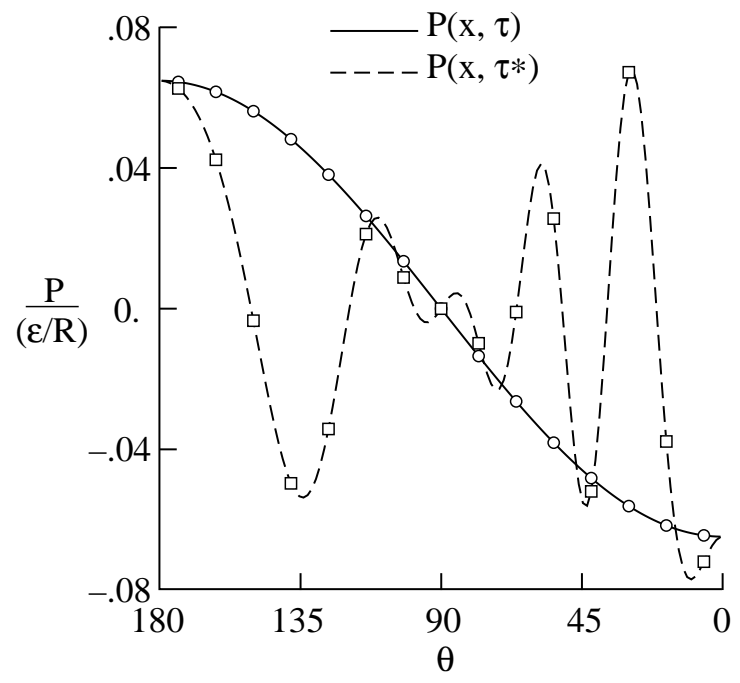

Fig. 3. Nondimensional pressure on a meridian line of a spherical Kirchhoff integration surface plotted at a function of the source time $\tau$ (i.e., a single time step from the CFD solution) and as a function of the retarded time $\tau *$, from [49]. 

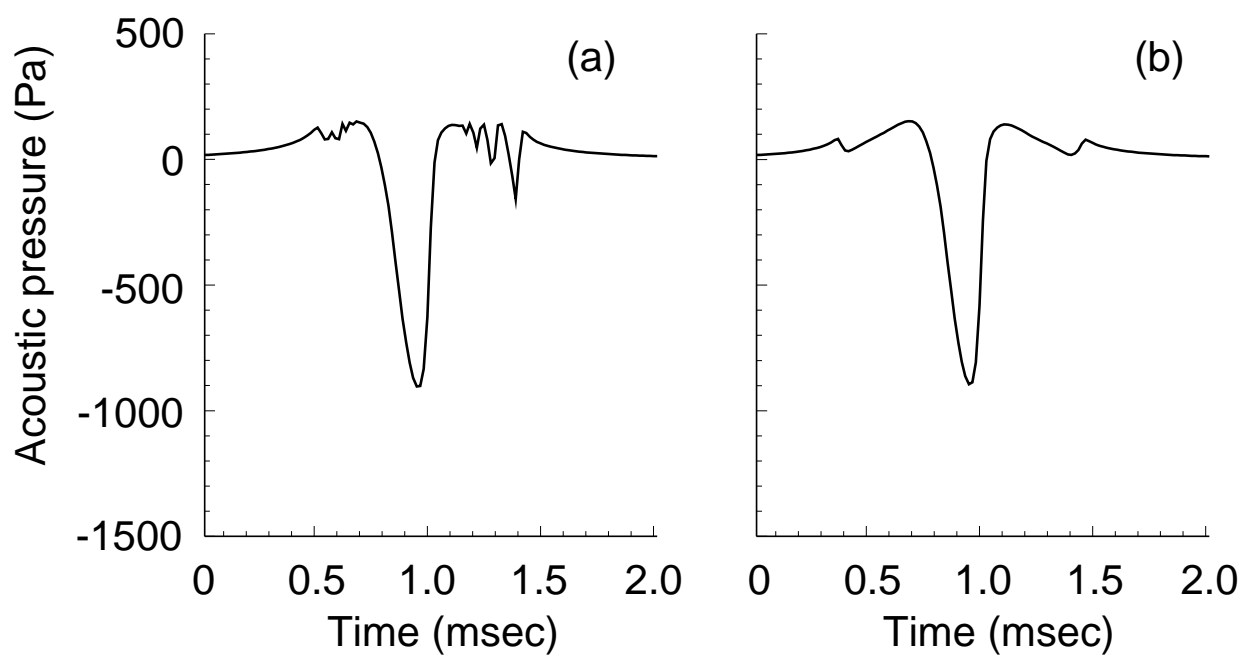

Fig. 4. Comparison of predicted solution with and without an adaptive quadrature algorithm. (a) No adaptation, mid-panel quadrature. (b) Adaptive quadrature. (Note that considerable panel size variation occurred in this computation.) 


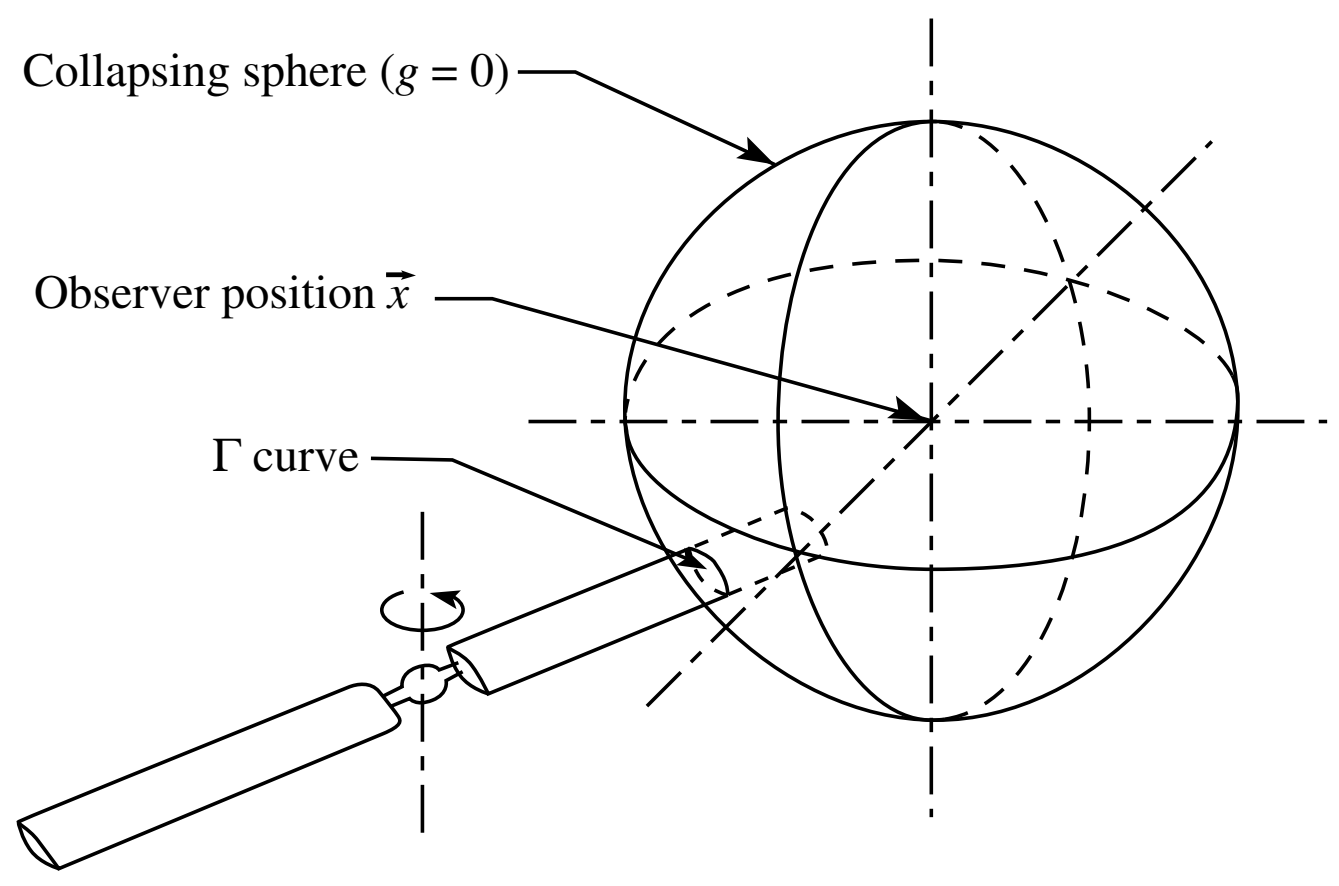

Fig. 5. Schematic of collapsing sphere, rotor blade and their intersection. 

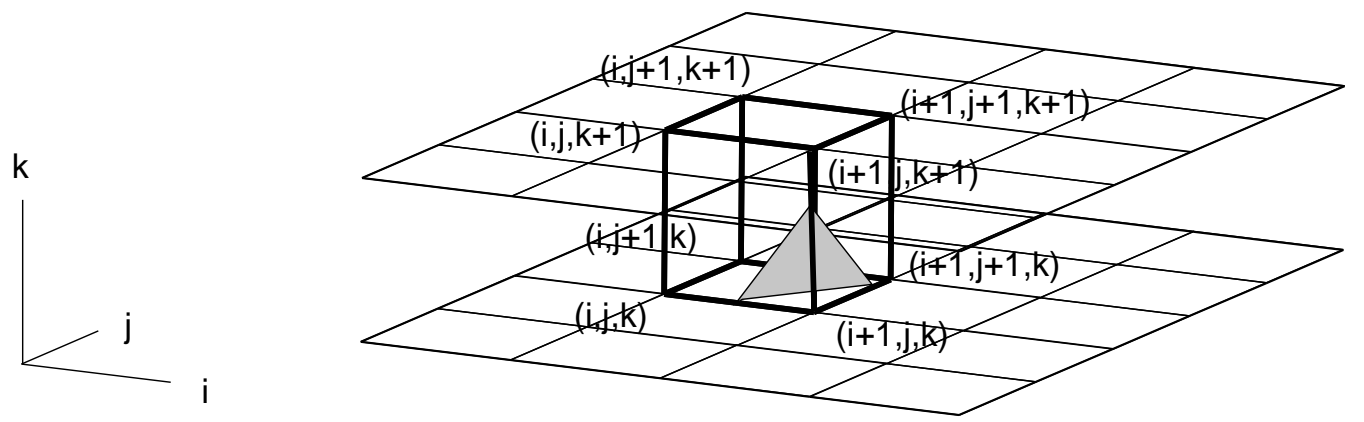

Fig. 6. Schematic of logical grid, marching cube and triangular piece of surface in marching cube. 


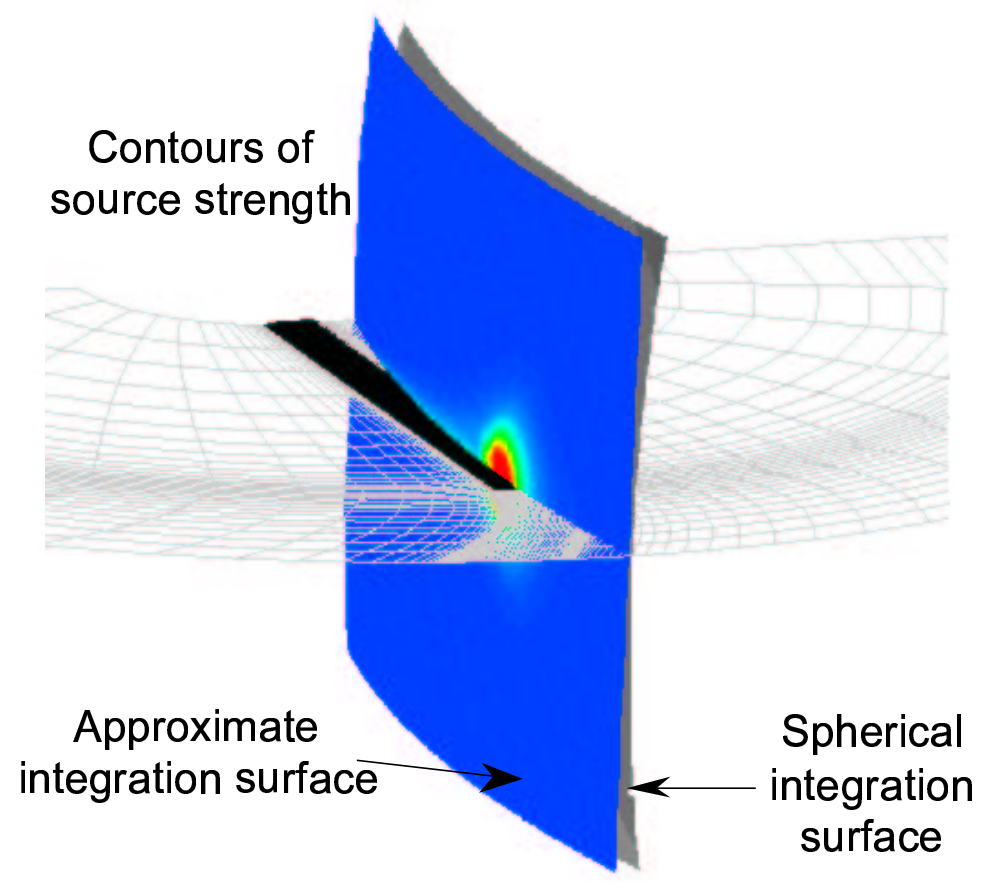

Fig. 7. Far-field approximation of the collapsing sphere. Although not shown, the observer is three rotor radii to the right of the rotor blade. 

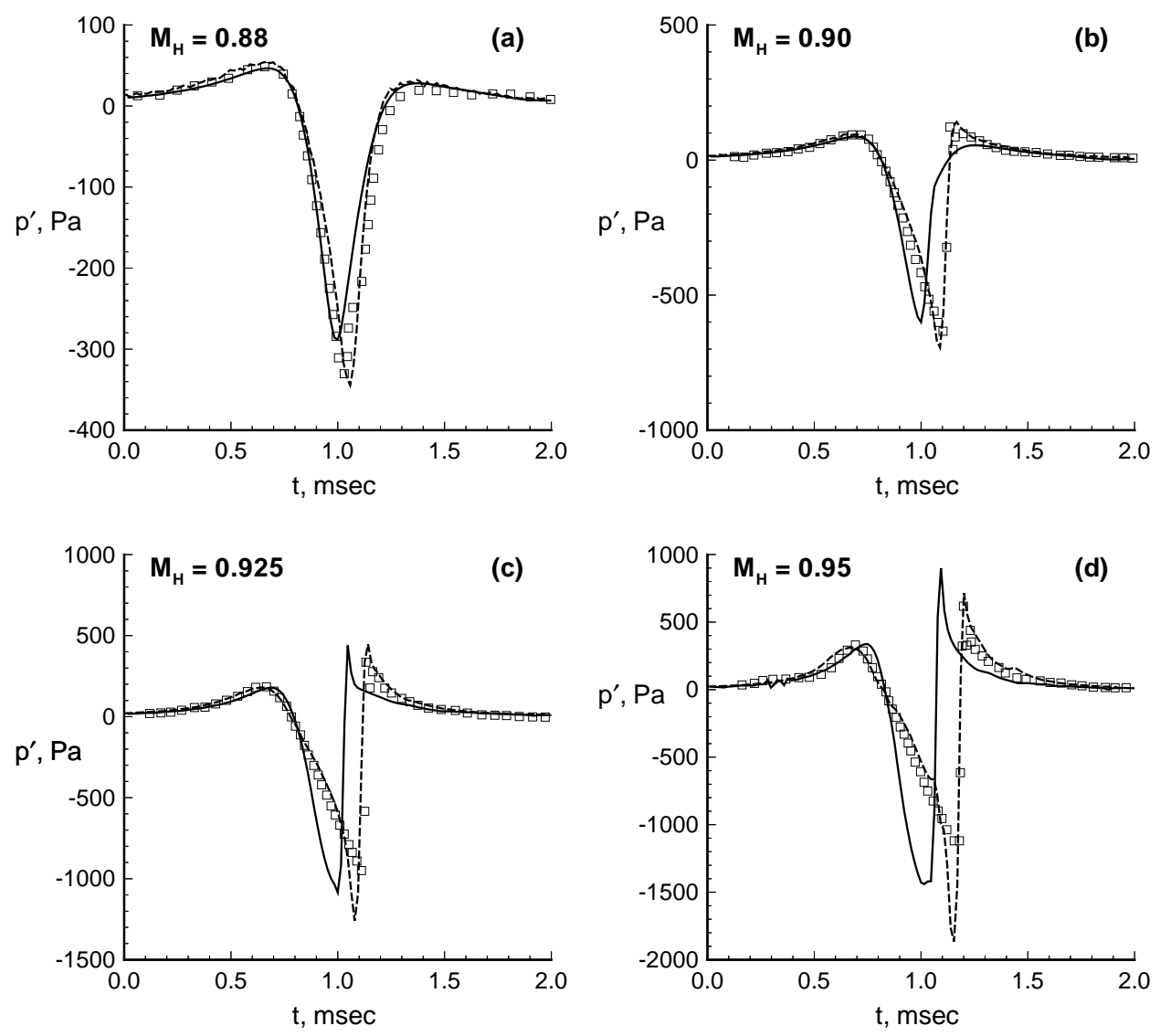

Fig. 8. Comparison of WOPWOP $+(-)$ and WOPWOP2+ $(---)$ predicted acoustic pressure with experimental data [65] () for hovering model UH-1H rotor. Quadrupole grid in WOPWOP+ prediction extended almost to sonic circle and in WOPWOP2+ predictions extended $1.86 R$ beyond the rotor tip. 

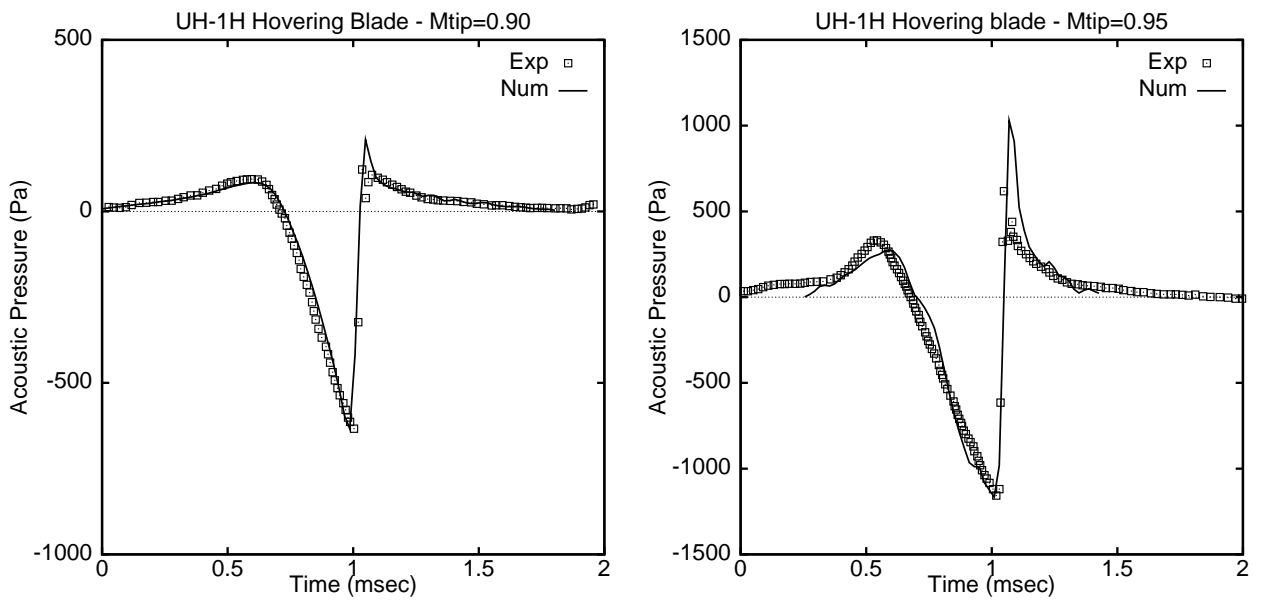

Fig. 9. Comparison of predicted acoustic pressure (-) [62] with experimental data [65] (ם) for hovering model UH-1H rotor. 


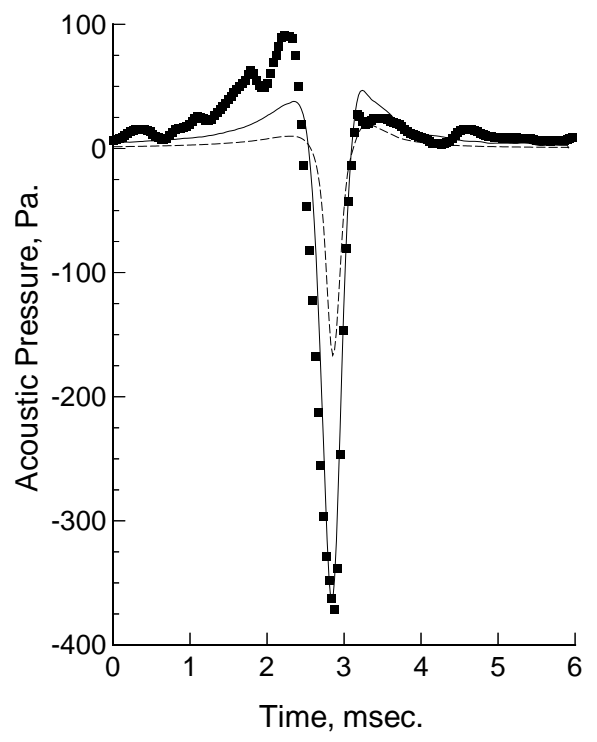

Fig. 10. Contemporary design, four-blade model rotor operating in forward flight; $\mu=0.32$ and $M_{A T}=0.933$. experimental data; $(-)$ predicted acoustic pressure; (- - - )quadrupole component of predicted acoustic pressure. 


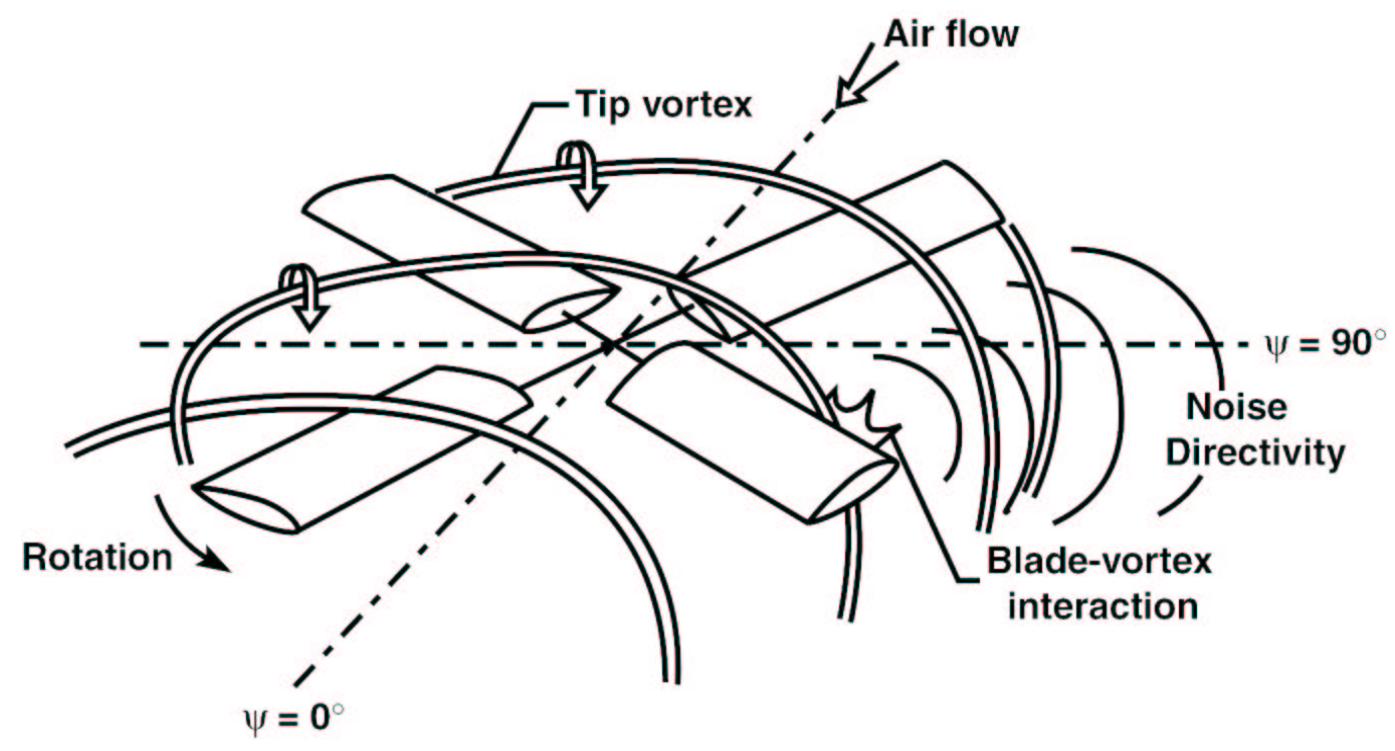

Fig. 11. Schematic of blade-vortex interaction. 

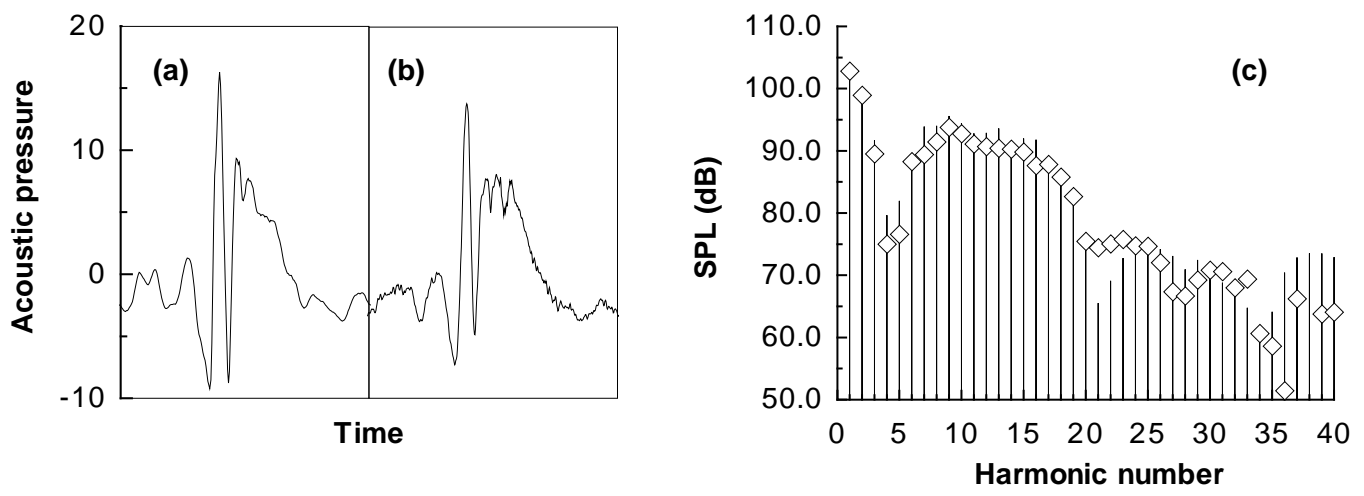

Fig. 12. Comparison of measured and predicted noise for a four-bladed Sikorsky model rotor. The microphone location for this comparison was nominally $25^{\circ}$ below the rotor plane on the advancing side, $\psi=150^{\circ}, 1.5$ rotor radii from the rotor hub. This is a descent condition, $\mu=.152$ and $C_{T} / \sigma=.07$, which corresponds to figure 3 in ref. [66]. (a) Measured time history; (b) predicted time history; (c) spectral comparison, —-, measured; $\diamond$, predicted. 


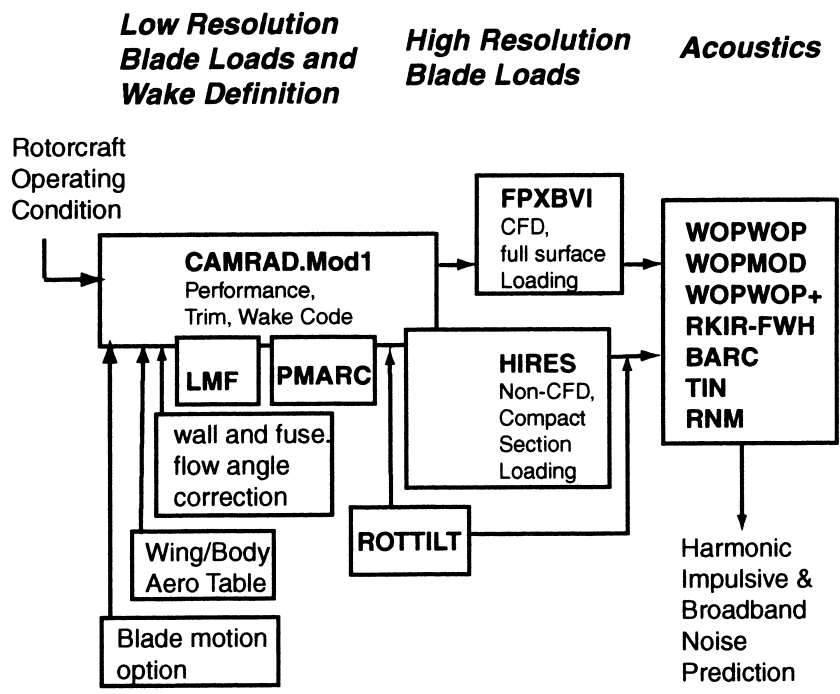

Fig. 13. Schematic of the TiltRotor Aeroacoustic Codes (TRAC) developed at the NASA Langley Research Center for helicopter and tiltrotor noise prediction [72]. 

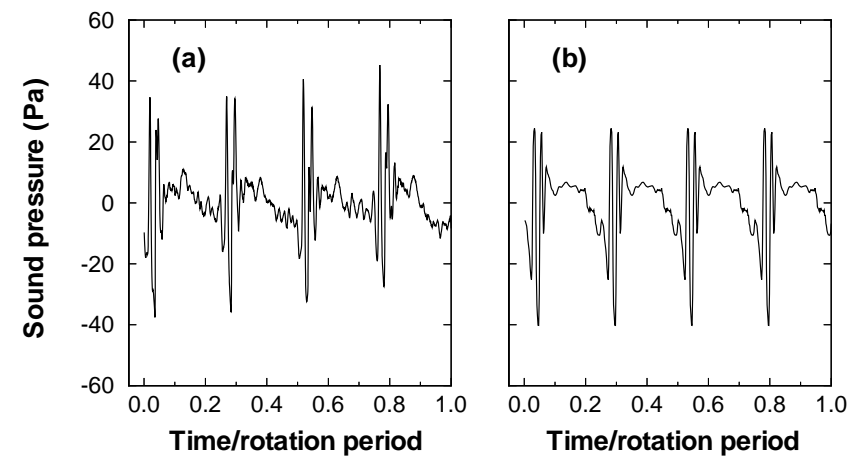

Fig. 14. A comparison of measured and predicted acoustic pressure time histories for the BO-105 HART model. For this case, $\mu=0.15, C_{T}=0.0044, M_{H}=0.64$ and the shaft angle $\alpha_{s}=5.3 \mathrm{deg}$. The observer is located $2.4 \mathrm{~m}$ below and $1.61 \mathrm{~m}$ right of the hub under the advancing side of the rotor. (This is figure 20(a) in ref. [72].) (a) experiment (b) prediction 

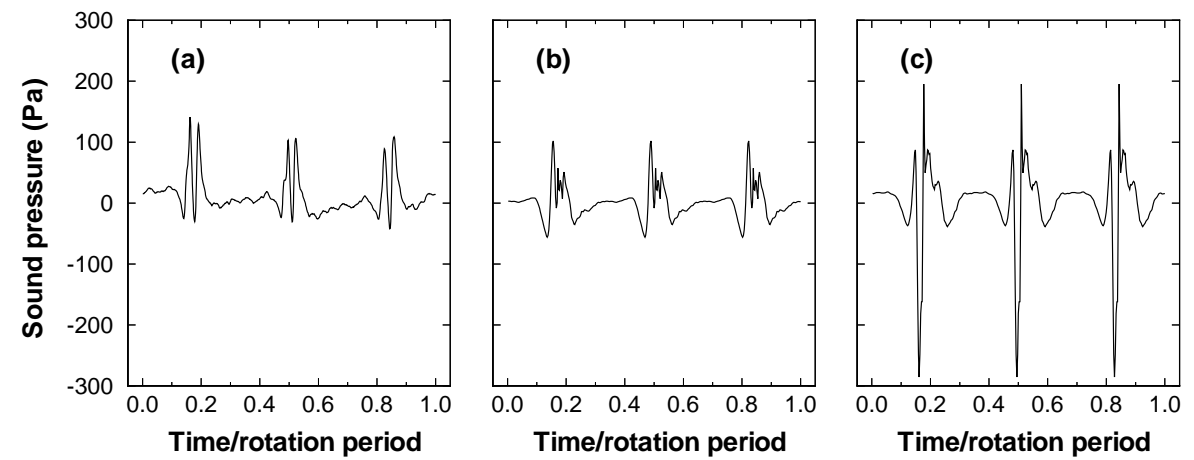

Fig. 15. Comparison of sound pressure measurement and predictions on the advancing side of a JVX tiltrotor model operating in hover. For this case, $\mu=0.17$, $C_{T}=0.00984, M_{H}=0.64$ and the shaft angle $\alpha_{s}=3.15 \mathrm{deg}$. The observer is located $1.512 \mathrm{~m}$ below, $1.372 \mathrm{~m}$ forward and $0.711 \mathrm{~m}$ right of the rotor hub. (This is a combination of figures 27 and 32 in ref. [72]. See refs. [72] and [73] for more details, including wind tunnel flow corrections.) (a) measured, (b) predicted-with roll-up model, (c) predicted-no roll-up model. 


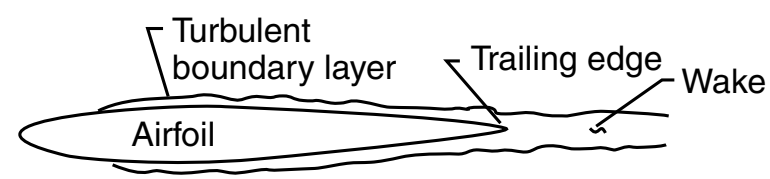

Turbulent boundary layer - trailing edge

(TBL-TE) noise
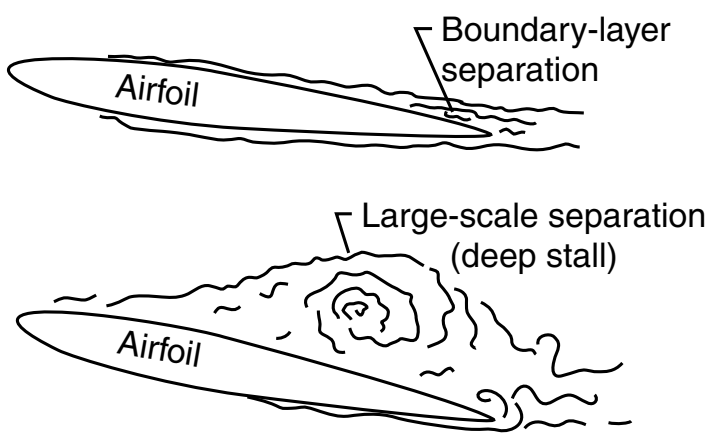

Angle dependence for TBL-TE noise

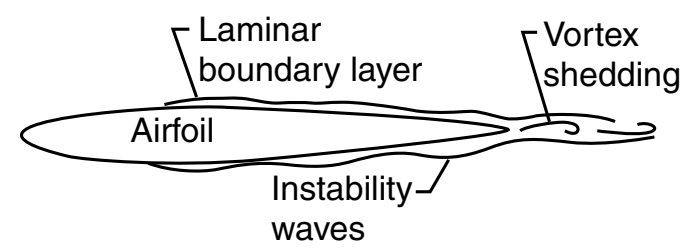

Laminar-boundary-layer-vortex shedding (LBL-VS) noise

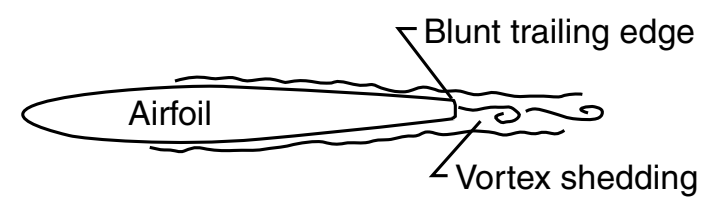

Blunt trailing edge (BTE) noise

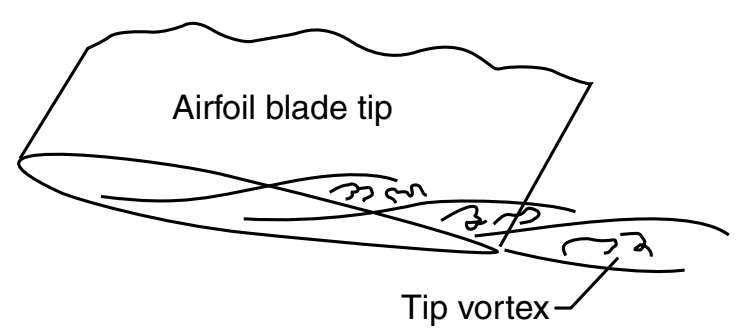

Tip noise

Fig. 16. An illustration of flow conditions producing blade self noise (from Brooks and Burley [85]). 


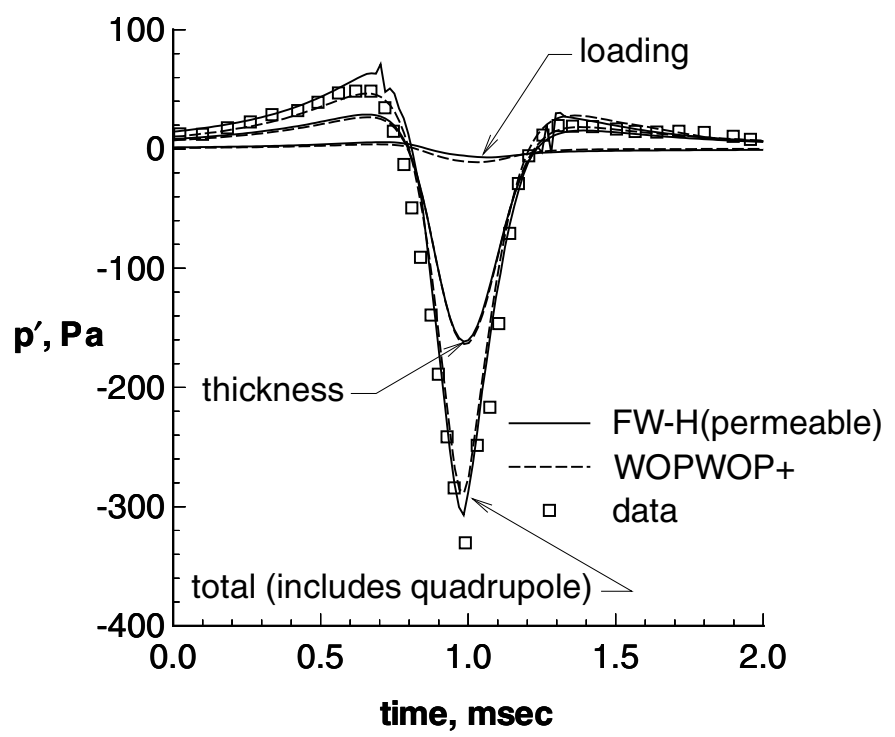

Fig. 17. Comparison of noise components predicted by the FW-H/RKIR and WOP$\mathrm{WOP}+$ codes for a hovering UH-1H model rotor $\left(M_{H}=0.88\right.$, inplane observer $3.4 R$ from rotor hub). 


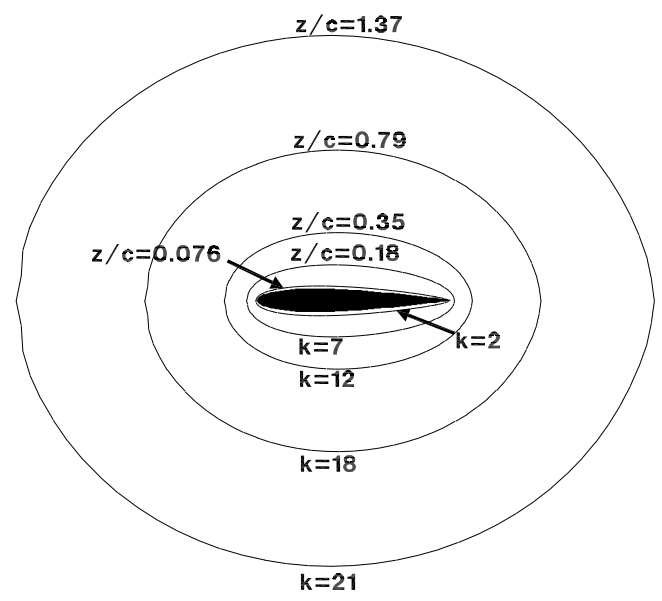

Fig. 18. Concentric cylindrical integration surfaces used for noise computation for a hovering rotor.

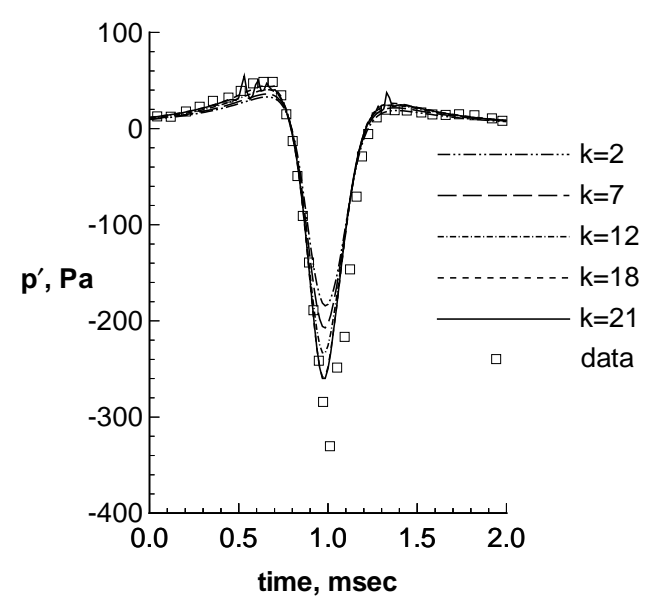

(a)

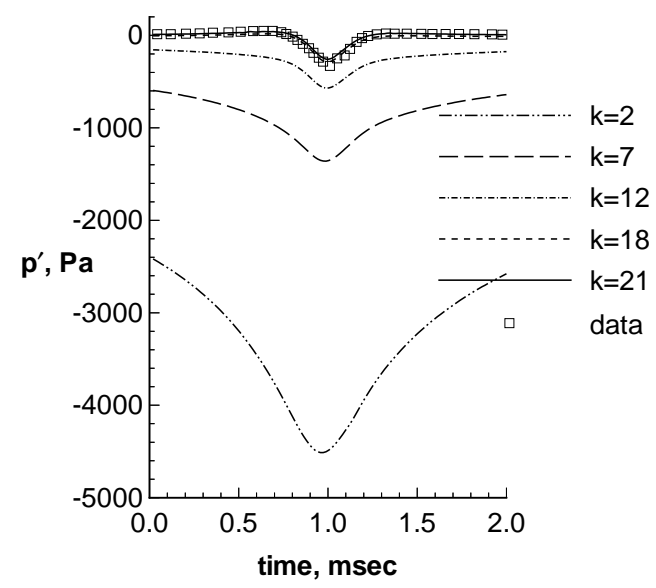

(b)

Fig. 19. Predicted acoustic pressure for various integration surface locations for an observer located $3.4 R$ from a $\mathrm{UH}-1 \mathrm{H}$ model rotor hovering at $M_{H}=0.88$. The experimental data $(\square)$ is from ref. 65. (a) FW-H prediction; (b) Kirchhoff prediction 


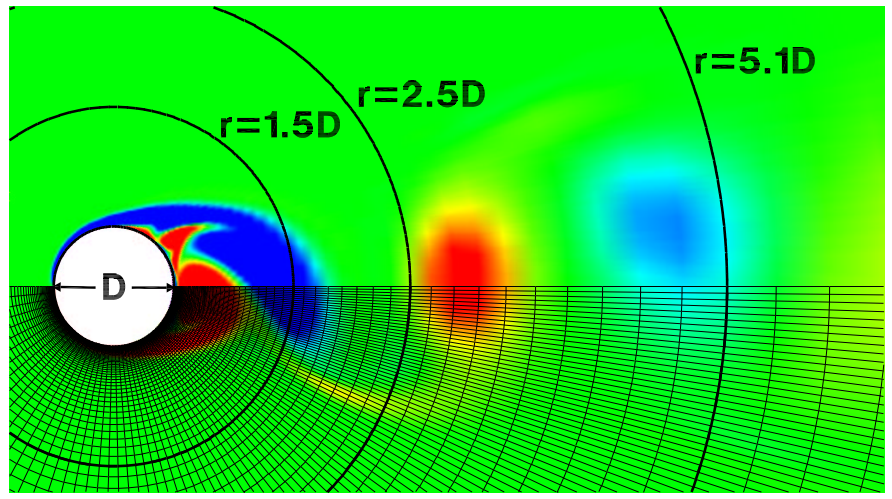

Fig. 20. Vorticity field computed from CFD. FW-H integration surfaces are at $r=0.5 D, r=1.5 D, r=2.5 D$ and $r=5.1 D$ 

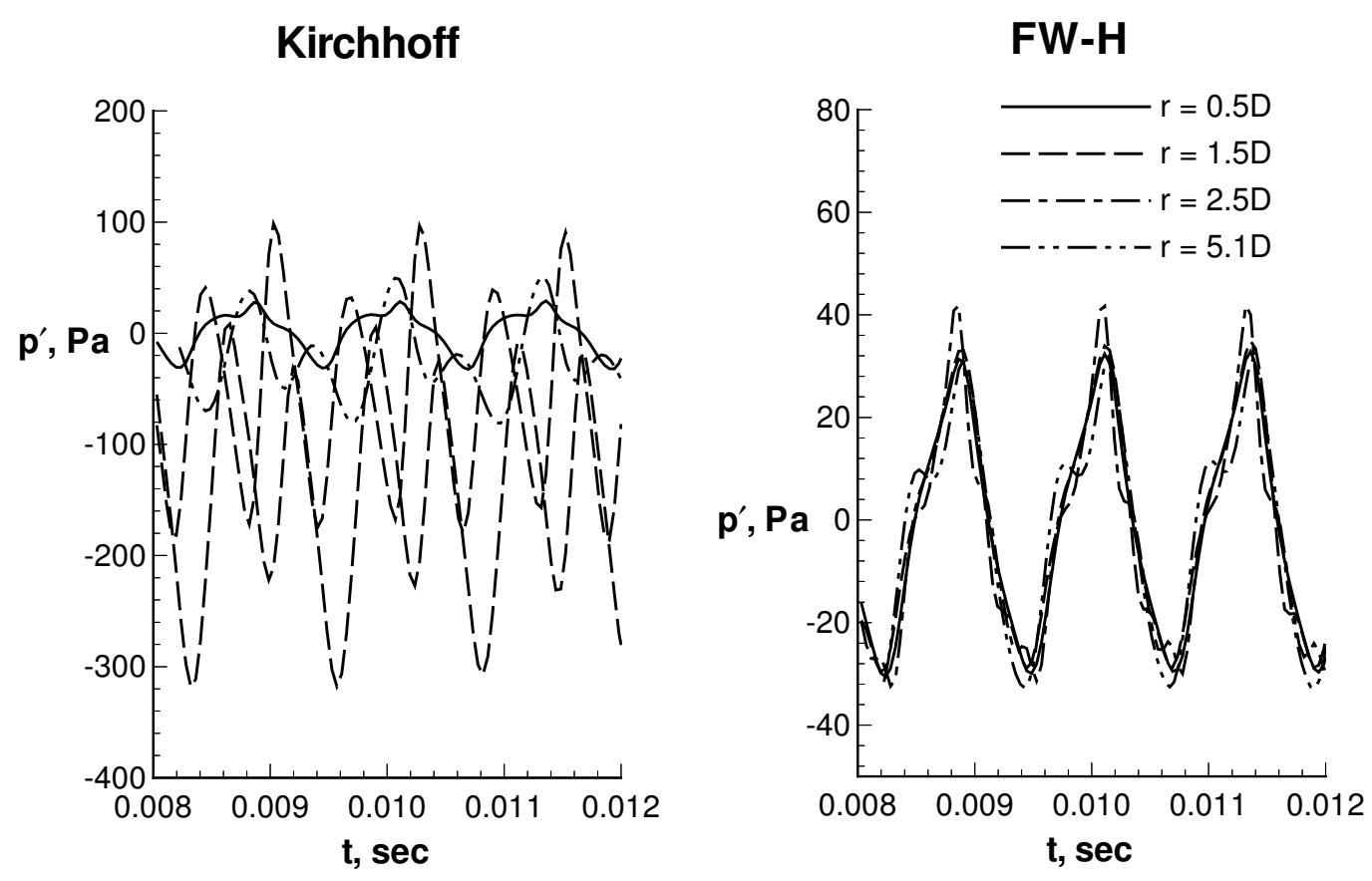

Fig. 21. Acoustic signals computed for various integration surfaces that correspond to those indicated in Figure 20. 


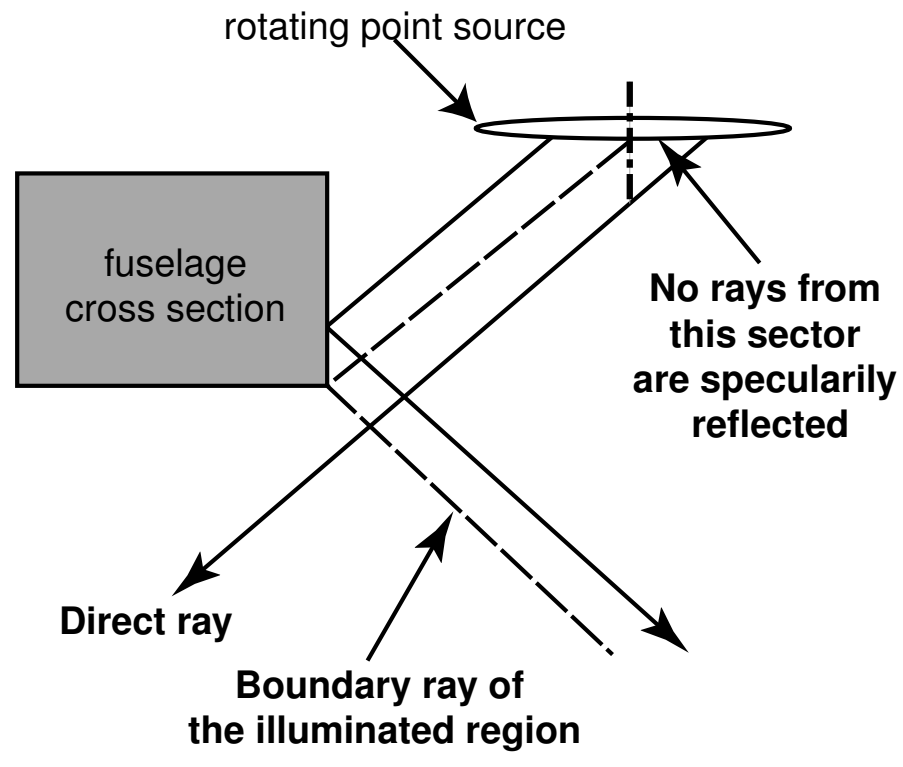

Fig. 22. Geometry of simple tiltrotor model which results in an additional impulse in the acoustic pressure time history [107]. 

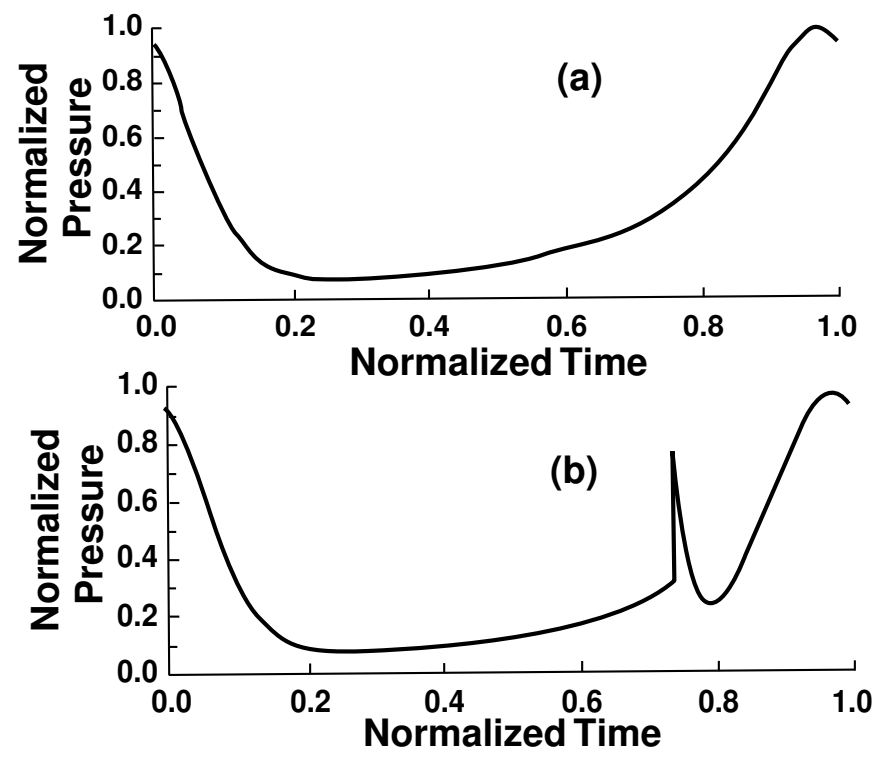

Fig. 23. Steady loading noise from a rotating steady point force near a rigid body (idealized tiltrotor model). (a) free-field, (b) near a rigid box. The source Mach number is $M=0.7$ and the observer is in far-field $45 \mathrm{deg}$. below and on the right side of the tiltrotor fuselage [107]. 
(a)

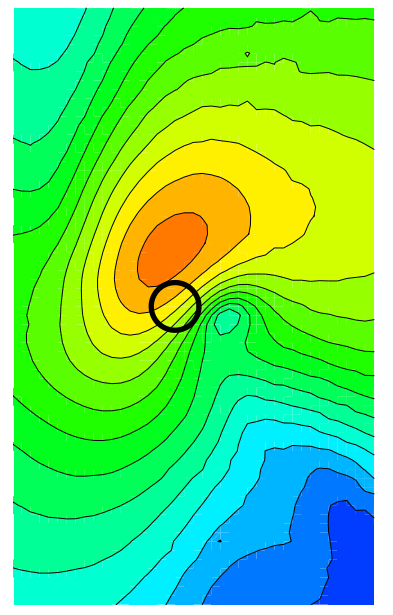

(b)

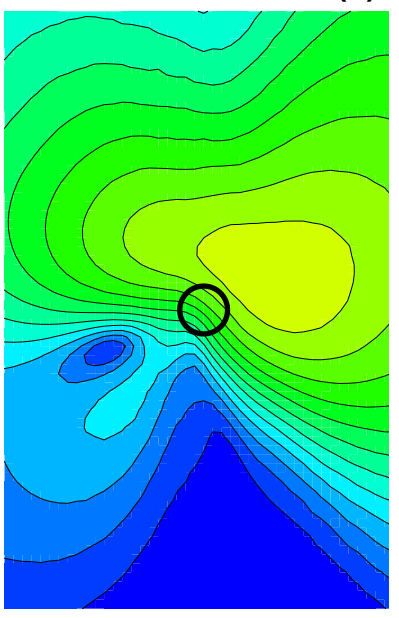

(c) $\quad d B$

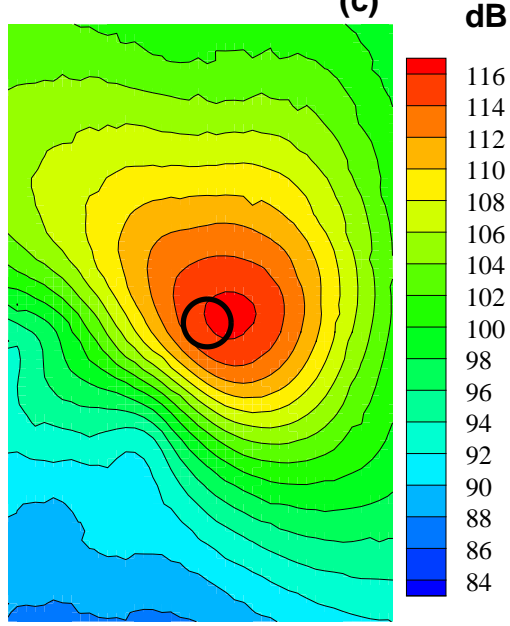

Fig. 24. Comparison of main-rotor noise overall sound pressure levels in level and turning flight [115]. (a) left turn, (b) level flight, (c) right turn; The flight direction is toward the top of the figure. 


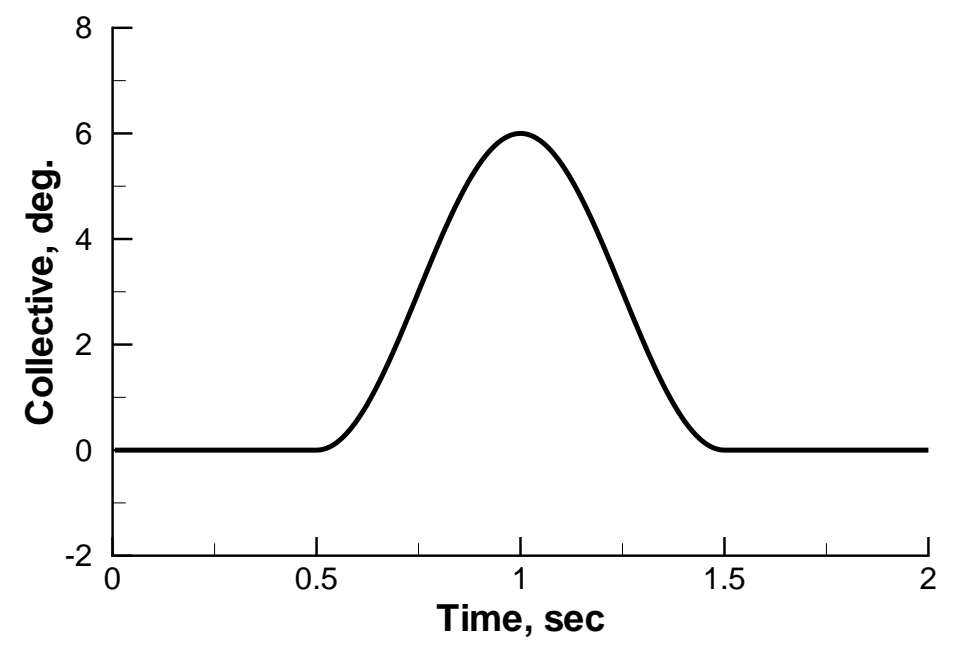

Fig. 25. Collective pitch control during arrested descent maneuver [55]. 


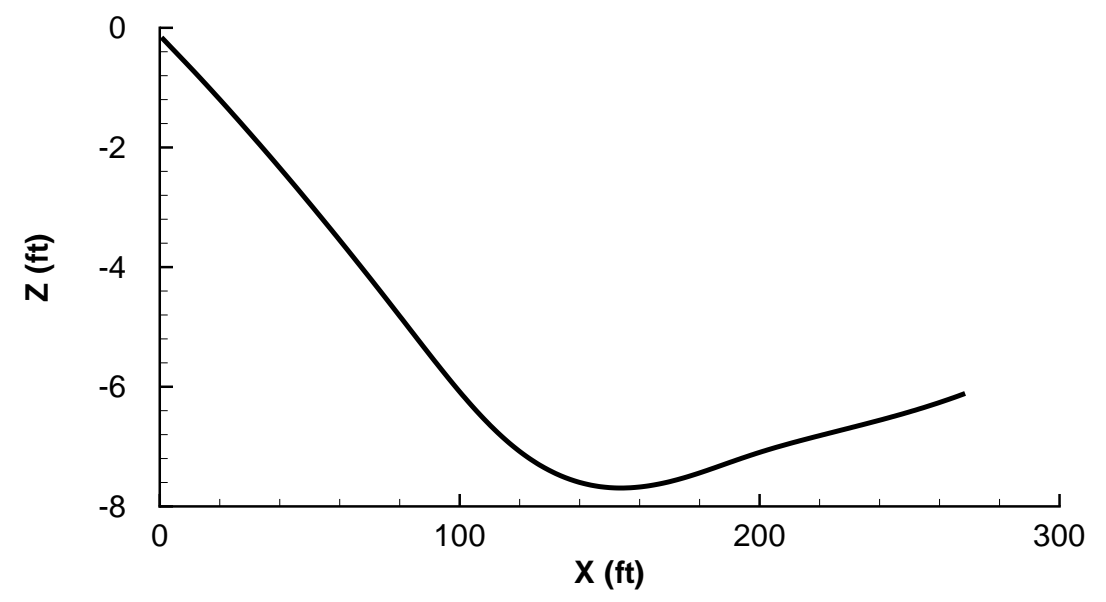

Fig. 26. Aircraft position during arrested descent maneuver [55]. The aircraft was at $\mathbf{x}=(0 ., 0 ., 0$. $)$ at $t=0 \mathrm{~s}$. 

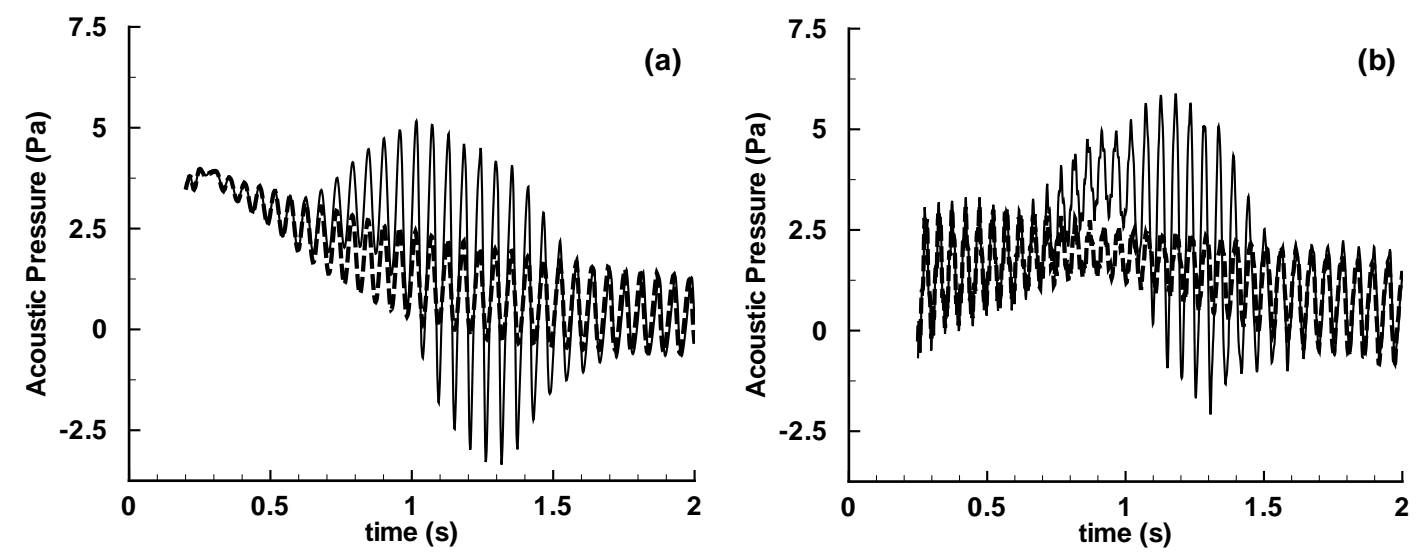

Fig. 27. Comparison of acoustic pressure time history for a three-degree steady descent (- - $)$ and an arrested descent maneuver ( - ). (a) observer located 100 ft below the helicopter at $t=0$ (from ref. [55])(b) observer located at $100 \mathrm{ft}$ below, $100 \mathrm{ft}$ to the right and $100 \mathrm{ft}$ forward of the helicopter at $t=0$ (from ref. [117]) 

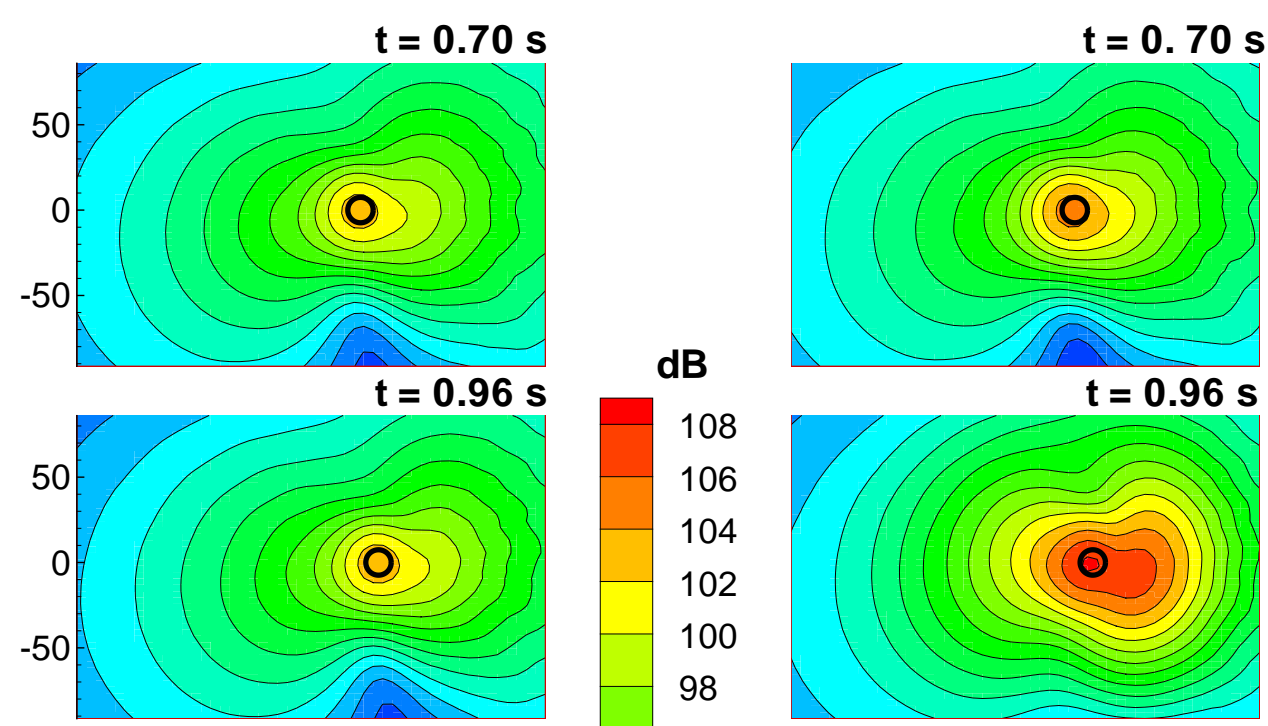

108

106

104

102

100

98

96
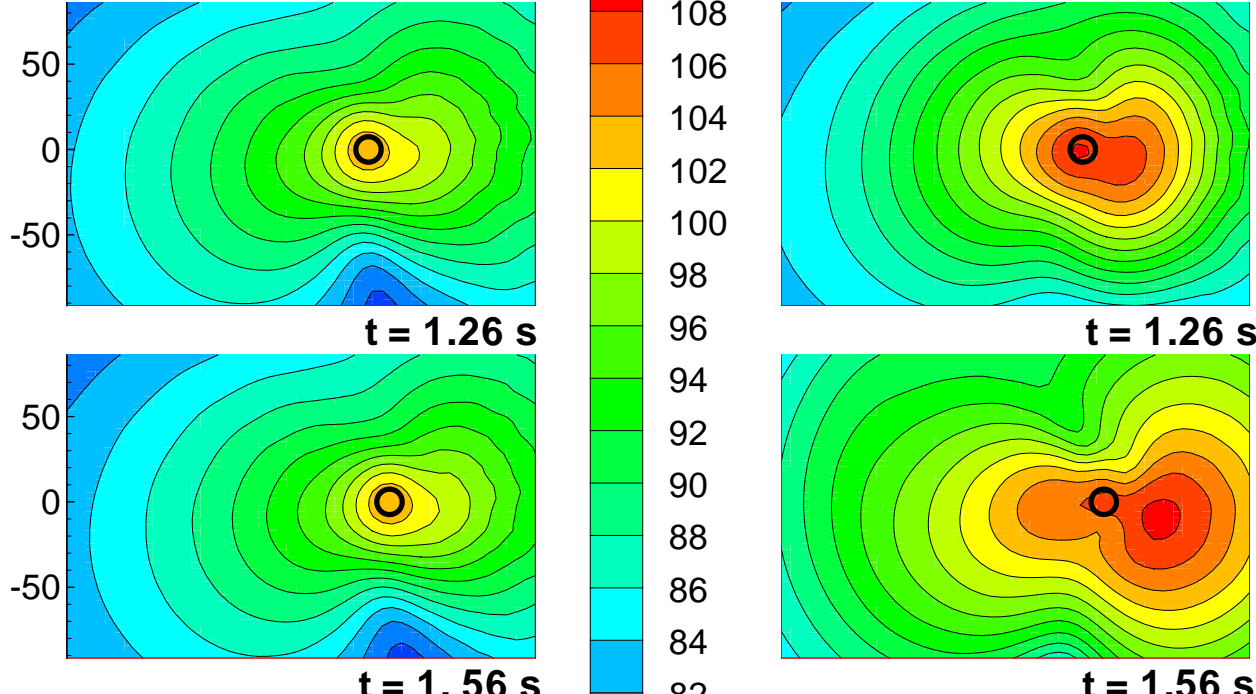

94
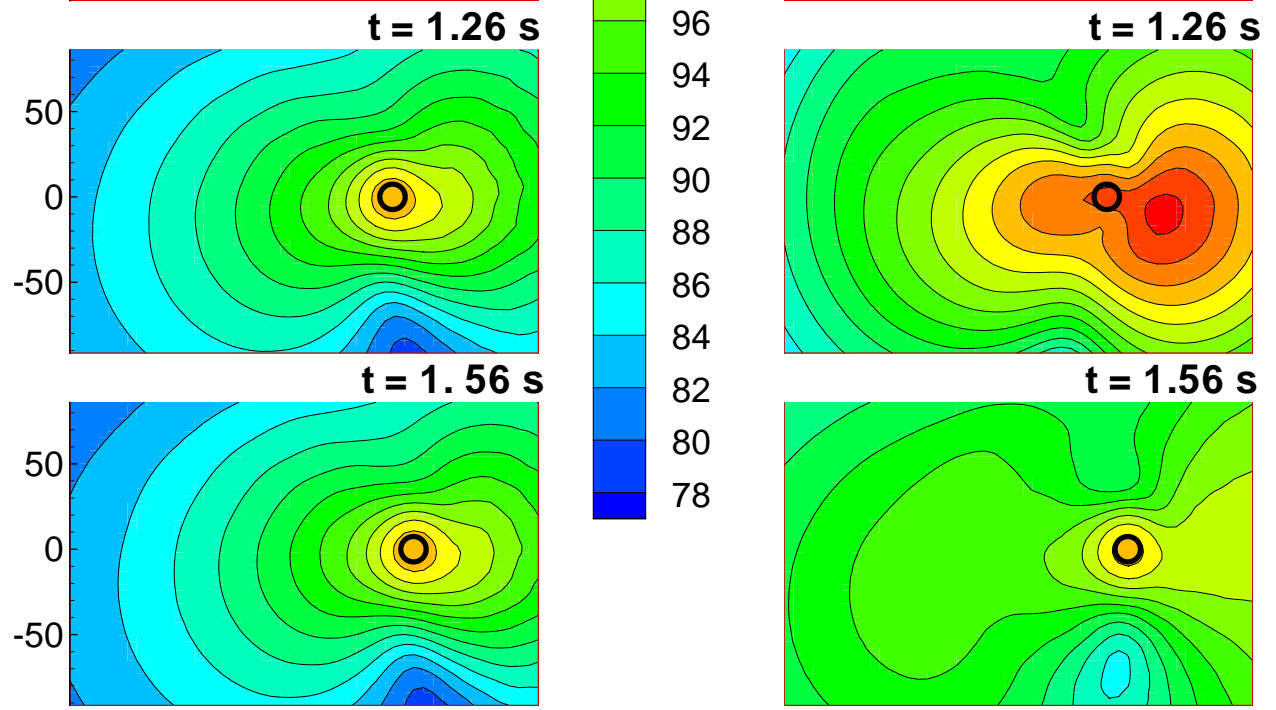

80

78
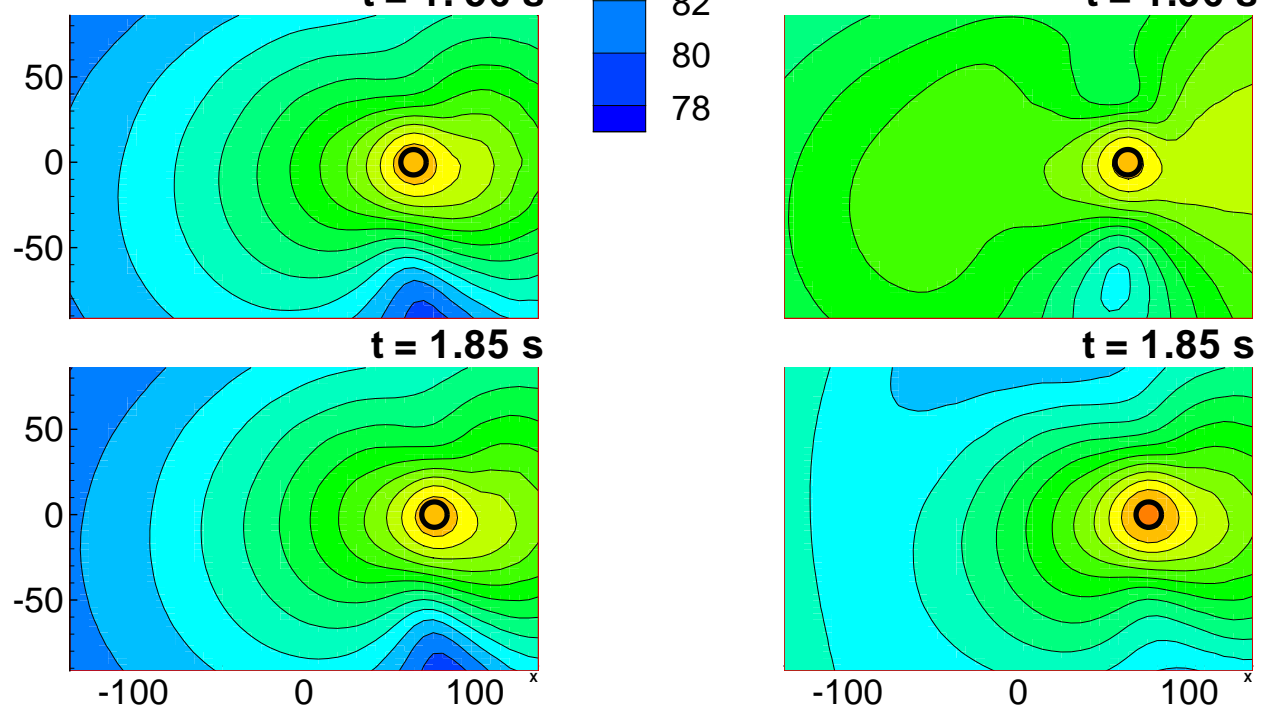

(a) steady 3 deg. descent

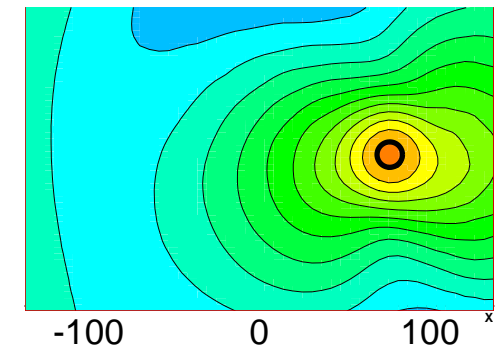

(b) transient - arrested descent

Fig. 28. Comparison of OASPL contours for the three-degree steady descent to the arrested descent maneuver as a function of time [117]. The circle is the projection of the main-rotor disk on the measurement plane. 